Linköping Studies in Science and Technology

Dissertation No. 1934

\title{
Doping and Density of States Engineering for Organic Thermoelectrics
}

\author{
Guangzheng Zuo
}

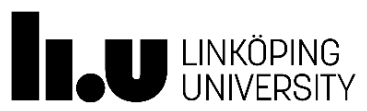

Complex Materials and Devices

Department of Physics, Chemistry and Biology (IFM)

Linköping University, SE-581 83 Linköping, Sweden 


\section{Cover Image}

Front image: doping impact on the density of states (DOS) of a single material. Dopants can induce charge carriers (white dots), while simultaneously the dopant ions (yellow dots) broaden the intrinsic DOS (white curve), adding a deep tail to the DOS (blue curve).

Back image: the formation of the total DOS for two blended materials (white and blue) and the positions of the Fermi energy and the transport energy with varying with material composition.

The cover image was designed by Guangzheng Zuo

Copyright $@$ C Guangzheng Zuo

Doping and Density of States Engineering for Organic Thermoelectrics

ISSN 0345-7524

ISBN 978-91-7685-311-5

Printed by LiU-Tryck. Linköping, Sweden, 2018 


\begin{abstract}
Thermoelectric materials can turn temperature differences directly into electricity. To use this to harvest e.g. waste heat with an efficiency that approaches the Carnot efficiency requires a figure of merit ZT larger than 1. Compared with their inorganic counterparts, organic thermoelectrics (OTE) have numerous advantages, such as low cost, large-area compatibility, flexibility, material abundance and an inherently low thermal conductivity. Therefore, organic thermoelectrics are considered by many to be a promising candidate material system to be used in lower cost and higher efficiency thermoelectric energy conversion, despite record ZT values for OTE currently lying around 0.25 .
\end{abstract}

A complete organic thermoelectric generator (TEG) normally needs both p-type and n-type materials to form its electric circuit. Molecular doping is an effective way to achieve p- and ntype materials using different dopants, and it is necessary to fundamentally understand the doping mechanism. We developed a simple yet quantitative analytical model and compare it with numerical kinetic Monte Carlo simulations to reveal the nature of the doping effect. The results show the formation of a deep tail in the Gaussian density of states (DOS) resulting from the Coulomb potentials of ionized dopants. It is this deep trap tail that negatively influences the charge carrier mobility with increasing doping concentration. The trends in mobilities and conductivities observed from experiments are in good agreement with the modeling results, for a large range of materials and doping concentrations.

Having a high power factor PF is necessary for efficient TEG. We demonstrate that the doping method can heavily impact the thermoelectric properties of OTE. In comparison to conventional bulk doping, sequential doping can achieve higher conductivity by preserving the morphology, such that the power factor can improve over 100 times. To achieve TEG with high output power, not only a high PF is needed, but also having a significant active layer thickness is very important. We demonstrate a simple way to fabricate multi-layer devices by sequential doping without significantly sacrificing PF.

In addition to the application discussed above, harvesting large amounts of heat at maximum efficiency, organic thermoelectrics may also find use in low-power applications like autonomous sensors where voltage is more important than power. A large output voltage requires a high Seebeck coefficient. We demonstrate that density of states (DOS) engineering is an effective tool to increase the Seebeck coefficient by tailoring the positions of the Fermi energy and the transport energy in $n$ - and p-type doped blends of conjugated polymers and small molecules.

In general, morphology heavily impacts the performance of organic electronic devices based on mixtures of two (or more) materials, and organic thermoelectrics are no exception. We experimentally find that the charge and energy transport is distinctly different in well-mixed and phase separated morphologies, which we interpreted in terms of a variable range hopping model. The experimentally observed trends in conductivity and Seebeck coefficient are reproduced by kinetic Monte Carlo simulations in which the morphology is accounted for. 


\section{Populärvetenskaplig sammanställning}

Termoelektriska material kan direkt omvandla temperaturskillnader till elektricitet. För att, med hjälp av till exempel spillvärme, kunna tillämpa detta fenomen med en effektivitet som närmar sig Carnotverkningsgraden krävs att ZT har ett meritvärde som är större än 1. Jämfört med inorganiska motsvarigheter har organisk termoelektricitet (OTE) flera fördelar såsom låg kostnad, möjlighet att tillämpa på stora ytor, flexibilitet, stor materialtillgång, och en naturligt låg värmeledningsförmåga. Därför anser många att OTE är en lovande kandidat att användas till billiga högeffektiva termoelektriska komponenter trots att rekordet för ZT-värden hos OTE ligger kring 0.25 .

En organisk termoelektrisk generator (TEG) behöver normalt både material av p-och n-typ för att bilda en fullständig elektrisk krets. Dopning av molekyler är ett effektivt sätt att åstadkomma material av p- och n-typ, men eftersom man använder sig av olika sorters dopning är det viktigt att förstå dopningsmekanismerna grundligt. Därför utvecklade vi en enkel men dock kvantitativ analytisk modell som vi jämförde med numeriska kinetiska Monte Carlo-simulationer för att ta reda på fenomenet bakom dopningseffekten. Resultaten visar att det uppstår tillstånd som sträcker sig djupt ner i den normalfördelade tillståndsdensiteten (DOS) på grund av Coulombpotentialerna hos de joniserade dopningsmolekylerna. Dessa tillstånd agerar som fällor för laddningar och har på så sätt en negativ inverkan på mobiliteten hos dessa. Trenderna för mobilitet och ledningsförmåga som observerats i experiment överensstämmer väl med de modellerade resultaten för ett stort antal olika material och dopningskoncentrationer.

I tillämpningar för TEG är det nödvändigt att de har en hög effektfaktor. Vi visar att dopningsmetoden starkt kan påverka de termoelektriska egenskaperna för OTE. Jämfört med traditionell bulkdopning kan sekventiell dopning uppnå en högre ledningsförmåga genom att bevara morfologin och därigenom kan effektfaktorn öka med över 100 gånger. För att uppnå TEG med hög uteffekt behövs förutom en hög effektfaktor även en betydande tjocklek hos det aktiva lagret. Vi visar att användning av sekventiell dopning är ett enkelt sätt att tillverka komponenter med multi-lager utan att försämra effektfaktorn nämnvärt.

Utöver att med hög effektivitet utvinna energi från stora värmekällor har OTE även användningsområden i lågeffektsapplikationer såsom självstyrande sensorer där spänning är viktigare än effekten. En hög utspänning kräver en hög Seebeckkoefficient. Vi visar att reglering av tillståndsfördelningen (DOS) är ett effektivt verktyg för att öka Seebeckkoefficienten genom att styra lägema av Fermi-energin och transportnivån hos n- och p-dopade blandningar av konjugerade polymerer och små molkeyler.

Överlag påverkar morfologin kraftigt prestandan hos organiska elektronikkomponenter som baseras på blandnigarn av två (eller fler) material, och detta gäller även inom termoelektronik. Via experiment upptäcker vi att laddnings- och energitransport är helt olika mellan välblandade morfologier och fasseparerade, vilket vi tolkar med en transportmodell där hopplängden kan variera. De experimentella trenderna hos ledningsförmåga och Seebeckkoefficient som vi observerat reproduceras i kinetiska Monte Carlo-simuleringar när morfologin tas i beaktande. 


\section{Included Papers}

[I] Impact of Doping on the Density of States and the Mobility in Organic Semiconductors

Guangzheng Zuo, Hassan Abdalla, Martijn Kemerink

Physical Review B 93, 235203 (2016)

[II] Molecular Doping and Trap Filling in Organic Semiconductor Host-Guest Systems Guangzheng Zuo, Zhaojun Li, Olof Andersson, Hassan Abdalla, Ergang Wang, Martijn Kemerink

The Journal of Physical Chemistry C 121, 7767-7775 (2017)

[III] High Seebeck Coefficient in Mixtures of Conjugated Polymers Guangzheng Zuo, Xianjie Liu, Mats Fahlman, Martijn Kemerink Advanced Functional Materials 1703280 (2017)

[IV] Range and Energetics of Charge Hopping in Organic Semiconductors Hassan Abdalla\#, Guangzheng Zuo ${ }^{\#}$, Martijn Kemerink Physical Review B 96, 241202(R) (2017)

${ }^{\#}$ Contributed equally to this work

[V] High Seebeck Coefficient and Power Factor in n-Type Organic Thermoelectrics Guangzheng Zuo, Zhaojun Li, Ergang Wang, Martijn Kemerink Advanced Electronic Materials 4, 1700501 (2018)

[VI] High Thermoelectric Power Factor from Multilayer Solution-Processed Organic Films

Guangzheng Zuo, Olof Andersson, Hassan Abdalla, Martijn Kemerink Applied Physics Letter 112, 083303 (2018)

[VII] Morphology Determines Conductivity and Seebeck Coefficient in Conjugated Polymer Blends

Guangzheng Zuo, Xianjie Liu, Mats Fahlman, Martijn Kemerink ACS Applied Materials \& Interfaces 10, 9638 (2018)

[VIII] General Rule for Trap Energies in Organic Semiconductors Guangzheng Zuo, ..., Martijn Kemerink To be submitted 


\section{Author contributions to the Papers}

To Paper [I], [II], [III], [V], [VI], [VII] and [VIII]:

Prepared the samples, performed the simulations and most of the measurements, wrote the manuscript drafts and revised them together with the coauthors.

To Paper [IV]:

Performed most of the experiments, wrote parts of manuscript and revised them together with the coauthors. 


\section{Contents}

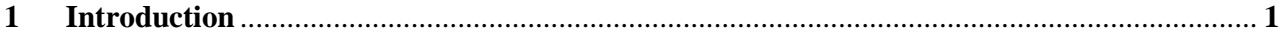

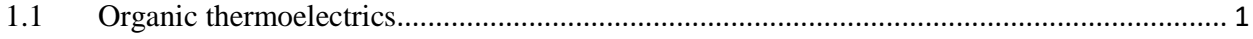

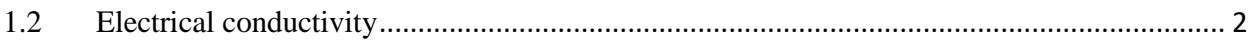

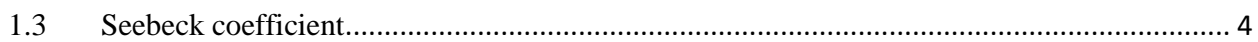

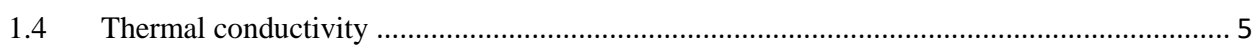

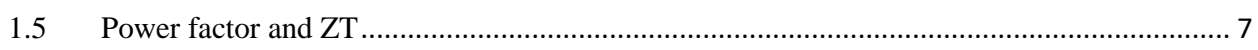

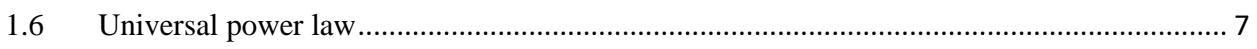

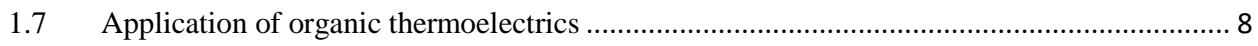

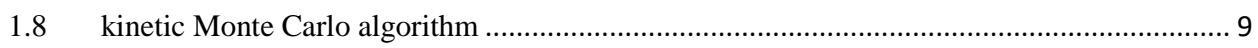

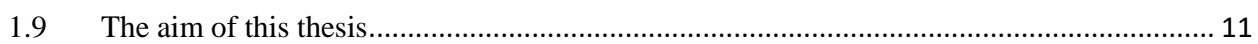

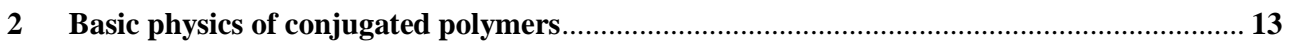

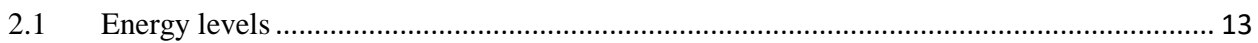

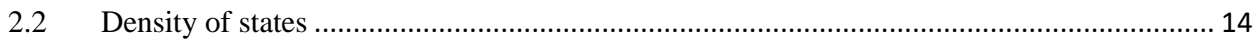

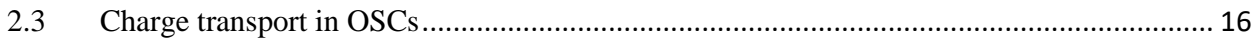

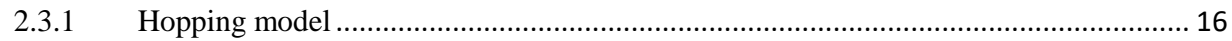

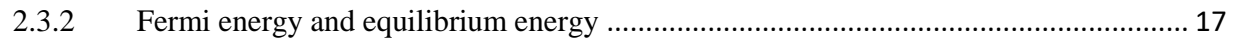

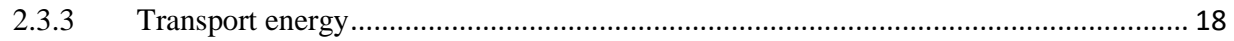

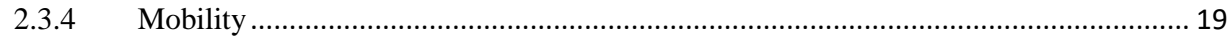

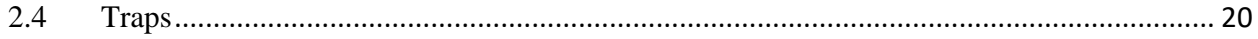

$3 \quad$ Doping.

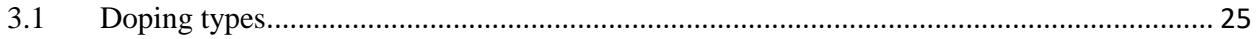

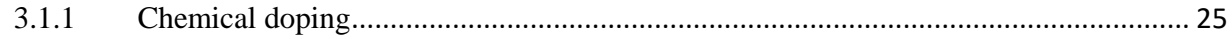

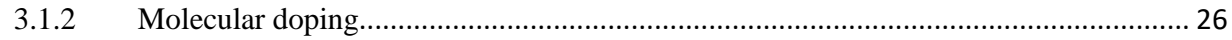

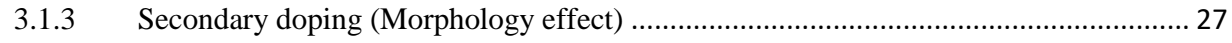

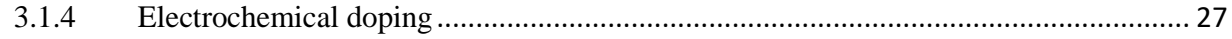

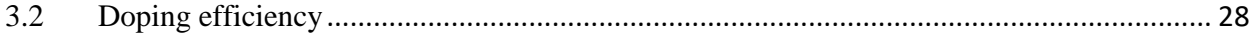

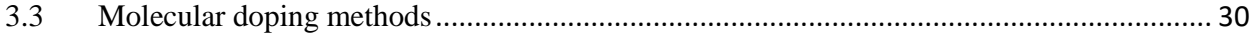

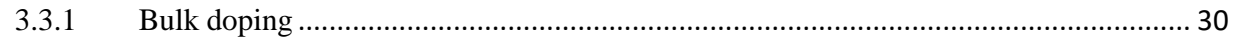

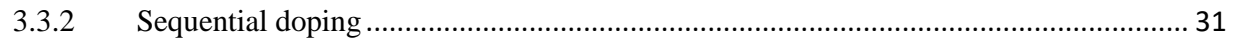

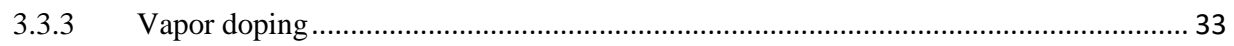

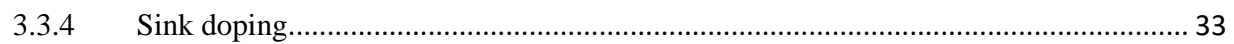

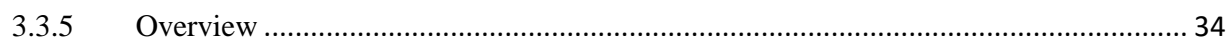

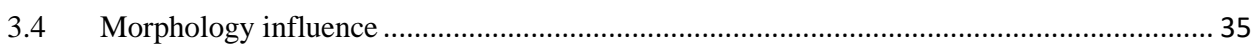




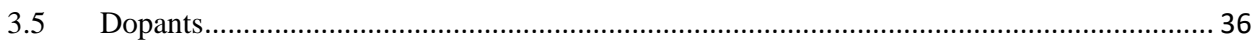

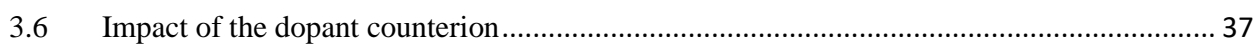

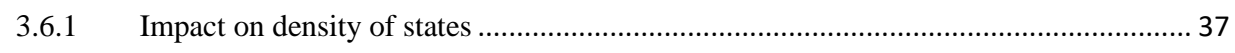

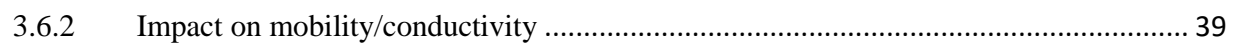

3.6.3 Impact on Seebeck coefficient and power law ...................................................... 40

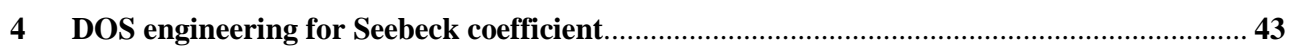

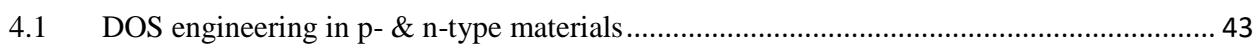

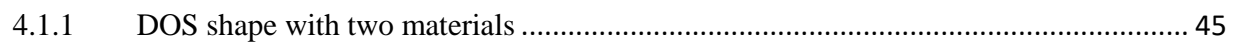

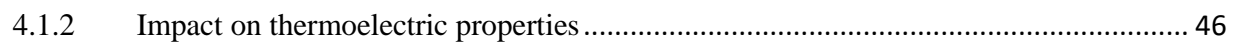

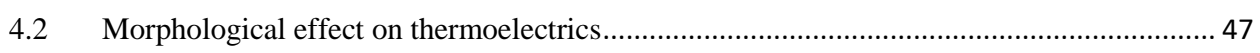

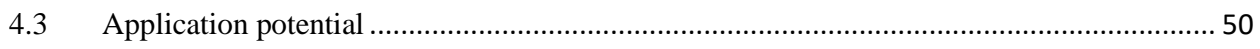

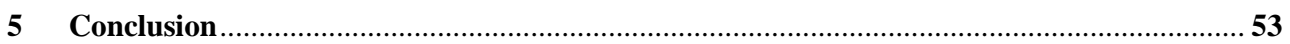

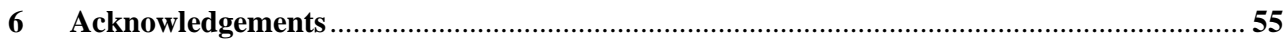

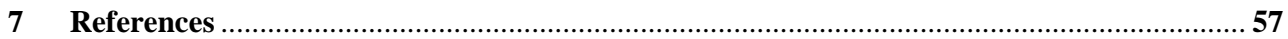

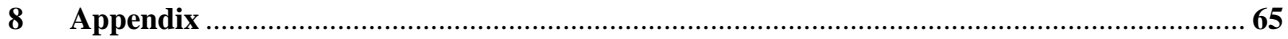




\section{Introduction}

\subsection{Organic thermoelectrics}

During the last two decades, especially after the year 2007, organic thermoelectrics have drawn more and more attention from the scientific community. ${ }^{1-5}$ Figure 1.1 shows the total number of publications and the sum of the number of times these have been cited in the field of organic thermoelectrics. Both are increasing dramatically each year. Furthermore, organic thermoelectrics are considered by many to be a promising candidate material system to be used in lower cost and higher efficiency thermoelectric energy conversion, ${ }^{6,7}$ despite record ZT values for OTE currently lying only around $0.25 .^{8}$
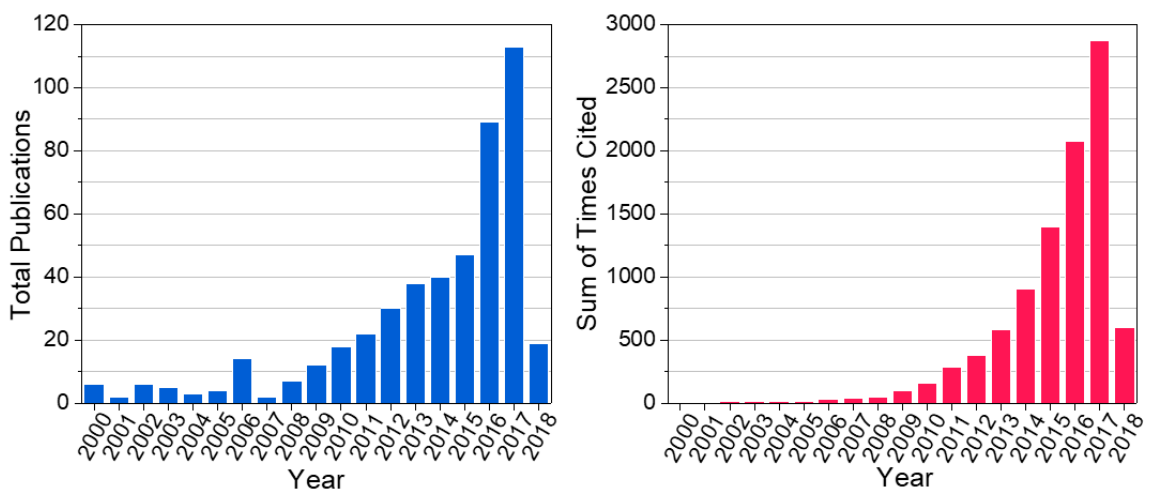

Figure 1.1 Publication report on 'organic thermoelectric materials' from Thomson Reuters Web of Science (Data taken on 12, March 2018).

With increasing demand for electricity, and exhausting usage of traditional energy sources like coal and fossil oil, a raring desire is burning to harvest electricity from renewable energy sources. Thermoelectric generators (TEG) can meet part of this need since they can turn temperature differences from waste heat or natural heat sources directly to electricity. ${ }^{9-12}$ The figure of merit ZT is typically used as evaluation criterion for a material's potential for efficient heat harvesting, as defined by:

$$
Z T=\frac{\sigma \cdot S^{2} \cdot T}{\kappa}
$$

Here, $\sigma$ is the electrical conductivity; $S$ is the Seebeck coefficient; $\kappa$ is the thermal conductivity and $T$ is the absolute temperature. 


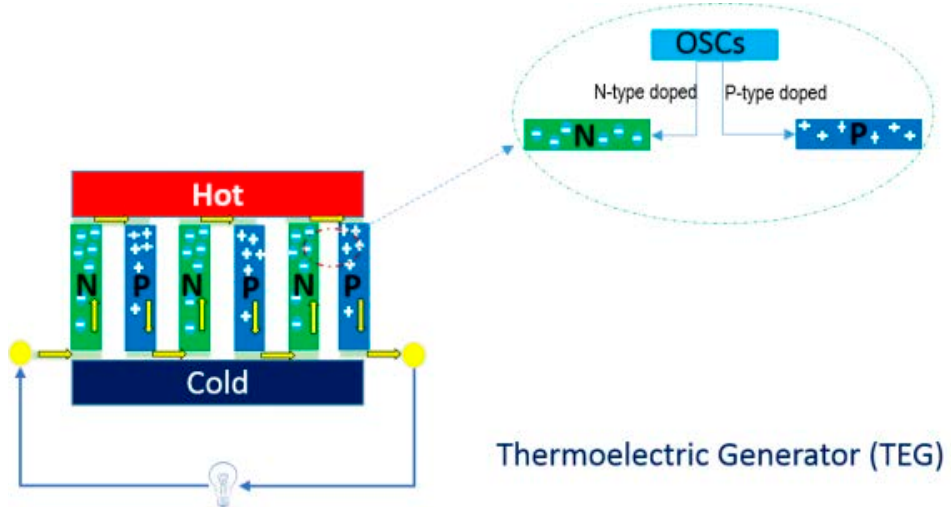

Figure 1.2 Structure of thermoelectric generator.

Inorganic materials like $\mathrm{Bi}_{2} \mathrm{Te}_{3}$ have achieved high performance with an $Z T$ around 1.0, ${ }^{13,14}$ whereas organic materials have reached only $\mathrm{ZT} \approx 0.25{ }^{8}$ However, compared with their inorganic counterparts, organic thermoelectrics (OTE) have several advantages, like compatibility with low cost, large-area deposition, flexibility, material abundance, non-toxicity and an inherently low thermal conductivity. ${ }^{15,16}$ Those features are fueling the striking development in organic thermoelectrics, considered by many to be a promising candidate for lower cost and higher efficiency thermoelectric energy conversion in the field of renewable energy.

In the remainder of this Chapter, the key concepts used throughout this thesis will be briefly introduced; more formal discussions and the contributions of this work to the field of organic thermoelectrics are given in Chapters 2-4.

\subsection{Electrical conductivity}

Electrical conductivity $(\sigma)$ can be derived from the current that flows through a rectangular piece of material due to an applied voltage by the following formula:

$$
I=\sigma \frac{w t}{l} V
$$

Here $I$ is the measured current, $V$ is the applied voltage, $l, t$ and $w$ are the length, thickness and width of the active layer, respectively. 


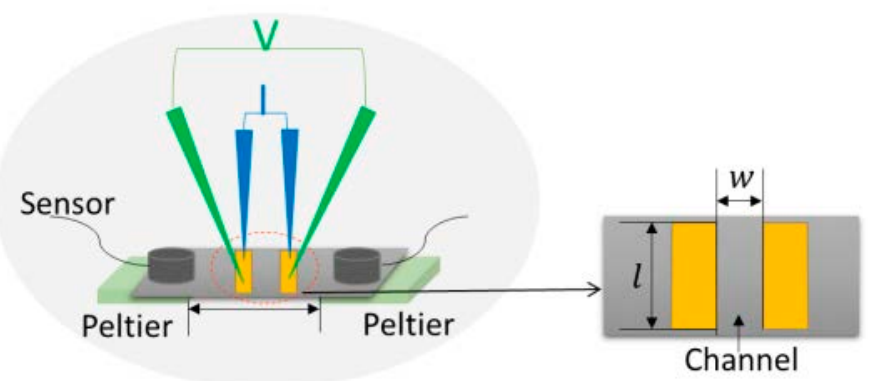

Figure 1.3 Illustration of geometry on electrical conductivity and Seebeck coefficient measurements and channel.

Undoped (intrinsic) organic electronic materials typically have quite low mobility $\mu$ and low intrinsic charge carrier density $n_{\text {int }}$ due to energetic disorder and the large bandgap. ${ }^{17}$ Those determine their low electrical conductivity in comparison to their inorganic counterparts, and this relationship enclosed by the formula:

$$
\sigma_{\text {int }}=n_{\text {int }} e \mu
$$

where $e$ is the elementary charge, the mobility $(\mu)$ is strongly dependent on the charge transport mechanism in disordered system and expressed with:

$$
\mu \propto \exp \left(\frac{E_{F}-E_{t r}}{T}\right)
$$

where $E_{F}$ is the Fermi energy; $E_{t r}$ is the transport energy. More details on this are discussed in Chapter 2.

Molecular doping is an effective way to improve the electrical conductivity by increasing the charge density and enhancing the conductivity. ${ }^{18-21}$ The relation between conductivity and dopant concentration is discussed further in Chapter 3. 


\subsection{Seebeck coefficient}

The Seebeck coefficient (also called thermopower, $S$ ) is one of the key quantities of thermoelectric materials. Based on the fact that in any non-constant density of states the Fermi energy is dependent on temperature as described by the Fermi-Dirac distribution, ${ }^{22}$ when a temperature difference $(\Delta T)$ is applied across the two side of a sample, a $E_{F}$ imbalance develops between the two sides, that is, $\Delta T \rightarrow \Delta E_{F}$. At zero current, this difference in chemical potential is compensated by an electrostatic potential difference $(\Delta V)$ between the two sides. The ratio between the different voltage and different temperature is called the Seebeck coefficient, as defined mathematically by:

$$
S=\frac{\Delta V}{\Delta T}
$$

The magnitude of the Seebeck coefficient is determined by the energy gap between the transport energy and the Fermi energy as shown as below. ${ }^{23}$ More discussion on this can be found in Chapter 2.

$$
S \propto \frac{E_{F}-E_{t r}}{T}
$$

To measure the Seebeck coefficient, a temperature gradient is typically created along the sample, using 2 Peltier elements. ${ }^{24}$ To simultaneously measure the local temperature, the sample is on each side sandwiched between a Peltier element and a Si-diode temperature sensor as seen in Figure 1.3. The applied field (voltage/inter-contact gap) can generate a counter-movement of charge carriers and create equilibrium such that the total current is equal to zero, i.e. the thermovoltage created by the temperature difference is equal to the applied voltage, $V_{t h}+S \Delta T=0$. This process is repeated for a number of temperature differences (in positive and negative direction) and the resulting $V_{t h}$ is plotted against the temperature difference between the two electrical contacts. This gives the Seebeck coefficient via the relation $S=\frac{\Delta V_{t h}}{\Delta T}$, i.e. the slope of said plot, as shown in Figure 1.4. 

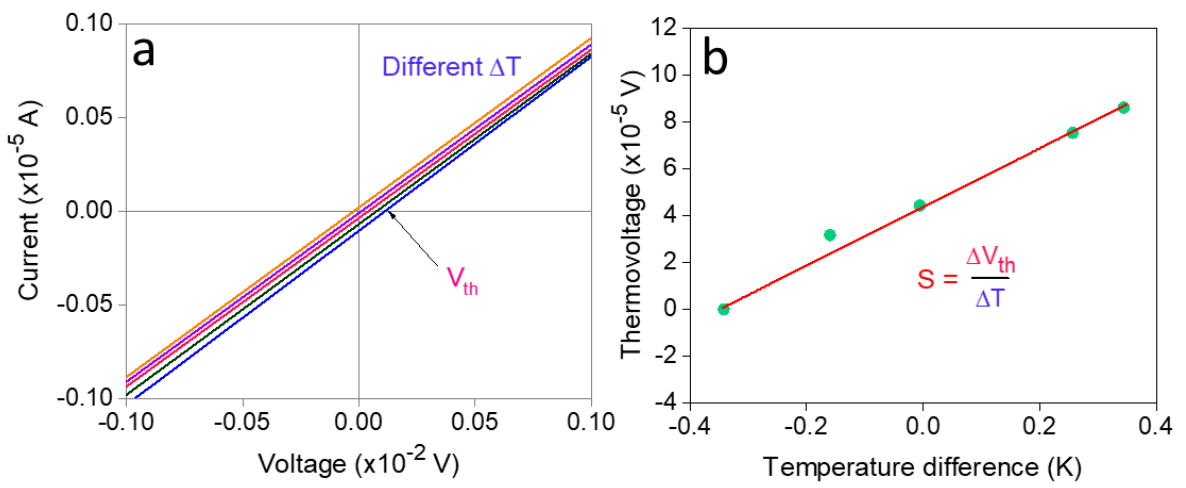

Figure 1.4 Illustration for derivation of thermo-voltage (a) from IV characteristic and calculation of Seebeck coefficient (b). Adapted with permission from Paper VI.

Normally, when the majority charge carriers in a (thermoelectric) material are electrons, the material is called n-type, while it is p-type when the majority charge carriers are holes. On the consequence of electrons and holes having different direction of motion under an applied field, the thermopower value has different sign for n-type (negative) and p-type (positive) thermoelectric material.

\subsection{Thermal conductivity}

Thermal conductivity is the parameter to describe the ability of a material to conduct heat. In solid materials, both charge carriers and lattice vibrations can contribute to heat transport. ${ }^{25,26}$ Accordingly, the thermal conductivity $(\kappa)$ can be defined as:

$$
\kappa=\kappa_{e}+\kappa_{l}
$$

With $\kappa_{e}$ the electronic contribution to $\kappa$ that is described by the Wiedemann-Franz law as

$$
\kappa_{e}=\left(k_{B} / e\right)^{2} L \sigma T
$$

where $k_{B}$ is the Boltzmann constant, and $L$ the dimensionless Lorenz factor; For simplicity, $L=$ $L_{0}$, with $L_{0}=\pi^{2} / 3$ the theoretical Sommerfeld value for a degenerate Fermi gas will be used in this work when needed. ${ }^{27}$ The lattice thermal conductivity $\kappa_{l}$ is the lattice vibrational contribution to $\kappa$ from phonons. Due to the disordered nature of organic materials, the value of $\kappa_{l}$ of conjugated polymers is typically quite low and suggested to have a more or less constant value of $\sim 0.2 \mathrm{~W} \mathrm{~m}^{-1} \mathrm{~K}^{-1}$. $^{28}$ 
Table 1.1 Summary of thermoelectric properties of various types of materials at $300 \mathrm{~K}$

\begin{tabular}{|c|c|c|c|c|c|}
\hline Materials & $\begin{array}{c}\text { Thermal } \\
\text { conductivity } \\
\left(\mathrm{W} \mathrm{m}^{-1} \mathrm{~K}^{-1}\right)\end{array}$ & $\begin{array}{c}\text { Seebeck } \\
\text { coefficient } \\
(\mu \mathrm{V} / \mathrm{K})\end{array}$ & $\begin{array}{c}\text { Electrical } \\
\text { conductivity } \\
(\mathrm{S} / \mathrm{m})\end{array}$ & ZT & Ref. \\
\hline $\mathrm{Si}$ & $\sim 150$ & $\sim 100$ & $\sim 1 \times 10^{5}$ & $\sim 0.01$ & Ref. $^{29}$ \\
\hline $\mathrm{Bi}_{2} \mathrm{Te}_{3}$ & $\sim 1.5$ & $\sim 225$ & $\sim 1 \times 10^{5}$ & $\sim 1$ & Ref. $^{30}$ \\
\hline PEDOT-Tos & $\sim 0.37$ & $\sim 200$ & $\sim 7 \times 10^{3}$ & $\sim 0.25$ & Ref $^{8}$ \\
\hline P3HT-F4TCNQ & $\sim 0.25$ & $\sim 287$ & $\sim 141$ & $\sim 0.01$ & Ref. $^{31}$ \\
\hline $\mathrm{CPs}$ & $\sim 0.2$ & $400 \sim 800$ & $10^{-5} \sim 10^{-7}$ & - & Ref. $^{32,33}$ \\
\hline
\end{tabular}

Table 1.1 summarizes the (total) thermal conductivity for typical inorganic and organic thermoelectric materials. The much lower $\kappa$ in OSC than in typical inorganics can be attributed to its intrinsic disordered structure that hampers phonon propagation. We note that the electronic contribution $\kappa_{e}$ becomes more dominant with increasing charge carrier density, i.e. in the high electrical conductivity regime as shown in Figure 1.5. ${ }^{34}$ At the same time, the electrical conductivity of organic thermoelectrics is much less than that of inorganics, which is also attributable to the mentioned disorder.

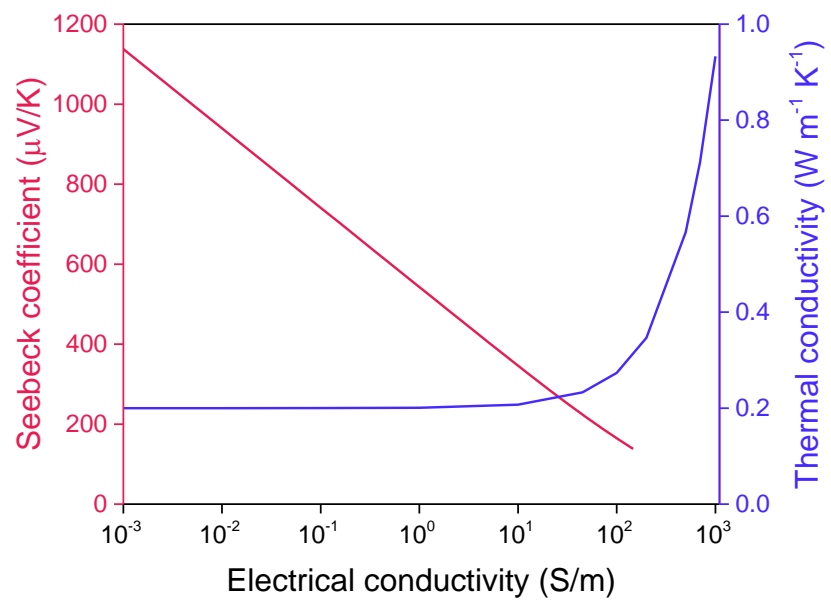

Figure 1.5 Seebeck coefficient and thermal conductivity vs. electrical conductivity at 300K. Seebeck coefficient calculated from the analytical model discussed in Chapter 2 and Paper I for a pristine OSC with parameters: inter-site distance $a_{N N}=1.8 \mathrm{~nm}$; Gaussian disorder $\sigma_{D O S}=$ $75 \mathrm{meV}$; inverse localization length $\alpha=5 \mathrm{e} 8 \mathrm{~nm}^{-1}$; attempt-to-hopping frequency $v_{0}=1 \mathrm{e} 13 \mathrm{~s}^{-1}$. Thermal conductivity is calculated from the above equations 1.7 and 1.8 .

For efficient thermoelectrics, the performance is a trade-off among the thermopower, electrical and thermal conductivity, and it is generally impossible to enhance all parameters 
simultaneously. Their relationship illustrated in Figure 1.4, that is, an increase in electrical conductivity in itself has an obvious advantage for thermoelectric efficiency (ZT), whereas the resulting significant decrease in $S$ and increase in thermal conductivity have considerable negative effects on the thermoelectric efficiency.

\subsection{Power factor and ZT}

As mentioned above, the figure of merit (ZT) is used as evaluation criteria for efficient energy conversion. ${ }^{35}$ Since the thermal conductivity in organic materials is quite low, thought to be about constant and is difficult to measure in thin films, one often uses the power factor (PF) to judge the ability of a material for heat harvesting.

$$
P F=\sigma \cdot S^{2}
$$

Recently, in p-type thermoelectric materials, Patel et al. showed that PBTTT vapor-doped with $\mathrm{F}_{4} \mathrm{TCNQ}$ on OTS-treated substrates can give a good power factor around $120 \mu \mathrm{W} / \mathrm{m} \cdot \mathrm{K}^{-2} \cdot{ }^{36}$ Bubnova et al. used the tosylate (Tos) to replace the polyanion (PSS) part in poly $(3,4-$ ethylenedioxythiophene) with polystyrene sulphonic acid (PEDOT:PSS), achieved a very high power factor over $300 \mu \mathrm{W} \mathrm{m}{ }^{-1} \cdot \mathrm{K}^{-2}$ with a $\mathrm{ZT}=0.25 .^{8}$ After a new air-stable n-type dopant, (4(1,3- dimethyl-2,3-dihydro-1H-benzoimidazol-2-yl)phenyl) dimethylamine (N-DMBI) was reported, ${ }^{37}$ there are many achievements in n-type thermoelectric materials. ${ }^{38-43}$ For instance, Huang et al. synthesized the small molecule A-DCV-DPPTT and used this kind of N-DMBI dopant and achieved record values for $\mathrm{PF}=95 \pm 10 \mu \mathrm{W} / \mathrm{m} \cdot \mathrm{K}^{-2}$ and $\mathrm{ZT}=0.11$ at room temperature. $^{38}$ Despite higher power factor having been achieved in p-type organic thermoelectrics at present, our experiments and simulations show that the n-type materials have more potential to achieve high $\mathrm{PF}$ and $\mathrm{ZT}$ values than p-type materials at room temperature. This will be further discussed in Chapter 4 .

\subsection{Universal power law}

Based on previous works on p-type doped materials, researchers found that the thermopower and the electrical conductivity are, for most materials, correlated with each other through an empirical power law relationship with slope $-1 / 4$ as shown in Fig. 1.6. ${ }^{44}$ 


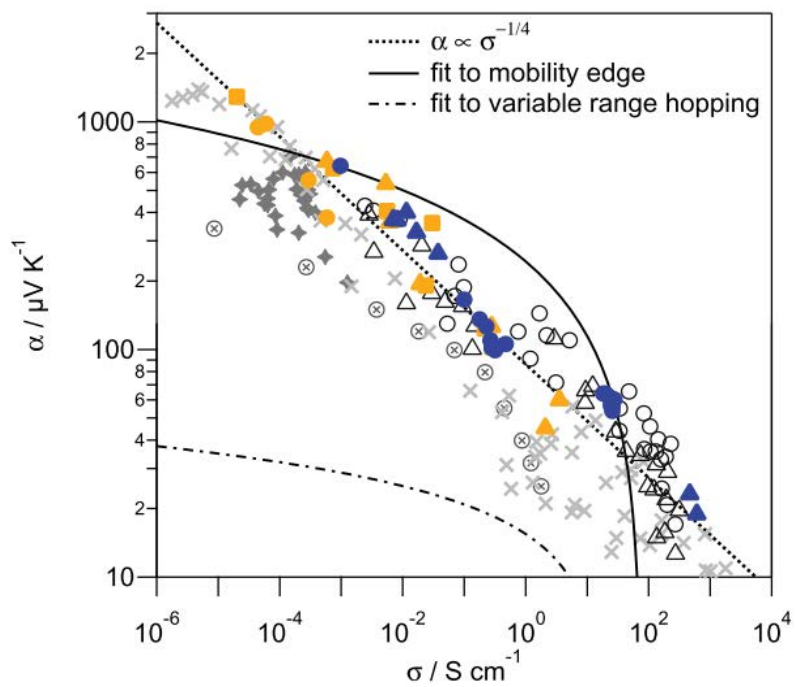

Figure 1.6 Thermopower vs. electrical conductivity data from experimental p-type doped OSCs (dots), empirical fitting (dotted line) and mobility edge model (solid line). Adapted with permission from Ref. $^{44}$.

In particular, Glaudell et al. suggested the following empirical relationship to express the relation between thermopower and conductivity: ${ }^{44}$

$$
S=\frac{k_{B}}{e}\left(\frac{\sigma}{\sigma_{\alpha}}\right)^{-1 / 4}
$$

where $\sigma_{\alpha}$ is an unknown conductivity constant that is independent of carrier concentration in the range covered. We found that n-type materials show a similar empirical thermopower trend (Paper V) and we provided a universal physical explanation for this behavior (Paper IV).

\subsection{Application of organic thermoelectrics}

A complete thermoelectric generator normally needs both p-type and n-type materials as a pair of legs to form its electric circuit as shown in Figure 1.2. A single TEG element only produces a quite low thermovoltage $(\Delta V=S \cdot \Delta T)$ of maximally a few millivolts that is insufficient to power electronic components since a typical requirement for the latter is one Volt. Therefore, an array of many pairs of legs is necessary. These are connected thermally in parallel but electrically in series. ${ }^{4}$

Depending on the desired scale of power, there are two different application directions for thermoelectric generators: type $\mathrm{I}$ is the high-power case, which needs a high $Z T$ to harvest (waste) heat at maximum efficiency. In addition, according to power $P \propto P F \cdot t$, having a significant active layer thickness $(t)$ is very important to output high power. ${ }^{31,45,46}$ Type II is the 
low-power case in which voltage is more important than power, e.g. to supply an autonomous sensor or a reflective LCD display with electricity. Here, power in the $0.01-1 \mathrm{~mW}$ range is sufficient and this type of TEG does not need high ZT, but is sensitive to cost and availability. ${ }^{23}$ Low cost implies flexible and easy fabrication, which is not compatible with a large number of legs in the TEG. Hence, a large output voltage $\Delta V=S \Delta T$, with $\Delta T$ the temperature difference over the TEG, per leg is needed. In other words, this type of TEG requires a high Seebeck coefficient and only a reasonable instead of an optimal conductivity.

\section{8 kinetic Monte Carlo algorithm}

Several numerical simulations included in the Papers employ a kinetic Monte Carlo algorithm (kMC) developed by Martijn Kemerink, to imitate real world experiments. The kMC simulations are used in section 3.6 and Paper I, II, III and VII, and describe the charge transport as thermally activated nearest neighbor hopping on a cubic lattice with site energies drawn from a Gaussian distribution. All Coulomb interactions, i.e. hole-hole and hole-ion, are explicitly accounted for, which is especially for high concentrations and doped systems as argued in Chapter 3.

\section{Hopping rate}

We use the Miller-Abrahams expression to quantify, with the least number of parameters, the nearest-neighbor hopping rate of a charge carrier from an initial state $i$ with energy $E_{i}$ to a final state $f$ with energy $E_{f}$ as

$$
v_{i f}= \begin{cases}v_{0} \exp \left(-\frac{\Delta E}{k_{B} T}\right), & \Delta E>0 \\ v_{0}, & \Delta E \leq 0\end{cases}
$$

where $v_{0}$ is the attempt to hop frequency and $\Delta E=E_{f}-E_{i} \pm q \vec{r}_{i f} \cdot \vec{F}+\Delta E_{C} \cdot \vec{F}$ is the external electric field, $\vec{r}_{i f}$ the vector connecting initial and final sites, and $q$ the positive elementary charge. The $+(-)$ sign refers to electron (hole) hopping. The term $\Delta E_{C}$ is the change in Coulomb energy due to interactions with other mobile charges and ions.

\section{Density of states and site energies}

We take a Gaussian shaped density of states (DOS) in our kMC simulations, with an expression of $\operatorname{DOS}(E)$

$$
g_{i}(E)=\frac{N}{\sqrt{2 \pi} \sigma_{D O S}} \exp \left(-\frac{\left(E-E_{i}\right)^{2}}{2 \sigma_{D O S}^{2}}\right)
$$

More details on density of states and its influence from dopant are shown in section 3.6,

The site energy of a particle $i$ due to the Coulomb interactions with all $N-1$ other particles is

$$
E_{\text {site }}^{i}=E_{i}+\sum_{j \neq i}^{N} E_{C}^{i, j}
$$


with $E_{i}$ the single-particle site energy at site $i$ and $E_{C}^{i, j}$ the Coulomb potential at site $i$ due to particle $j$.

The corresponding density of occupied states (DOOS) then is calculated from the condition

$$
\operatorname{DOOS}(E)=\operatorname{DOS}(E) f_{F D}\left(E_{F}\right)
$$

Where

$$
f_{F D}\left(E_{F}\right)=\frac{1}{1+\exp \left(\frac{E-E_{F}}{k_{B} T}\right)}
$$

is the Fermi-Dirac distribution that is a function of the Fermi energy $\left(E_{F}\right)$ and the temperature.

Calculation of Seebeck coefficient, electrical conductivity, and mobility

In the simulations, the Seebeck coefficient is calculated as

$$
S=\int_{-\infty}^{\infty} d E \frac{\left(E-E_{F}\right) \sigma(E)}{\sigma T}
$$

where the conductivity $\sigma$ is related to the conductivity distribution function $\sigma(E)$ through

$$
\sigma=\int_{-\infty}^{\infty} d E \sigma(E)
$$

Defining an 'energy-transport energy' as

$$
E_{E, t r}=\int_{-\infty}^{\infty} d E \frac{E \sigma(E)}{\sigma T}
$$

yields back the expression $S=\frac{E_{F}-E_{E, t r}}{T}$ that is of the same shape as Eq. 1.6. We found that the difference between $E_{E, t r}$ and $E_{t r}$, that is used in Eq. 1.6, is small, as further discussed in the SI of Paper III.

The mobility and conductivity are derived from calculated current densities at a finite DC electric field using essentially the same expressions Eq. 1.2 and 1.3 as used for experiments. More details on mobility are discussed in section 2.3 and 3.6. 


\subsection{The aim of this thesis}

The work in this thesis aims to understand the influence of doping, density of states (DOS) and morphology on charge transport in disordered organic semiconductors, in particular on various physical properties like mobility, conductivity and Seebeck coefficient for organic thermoelectrics. To deepen physical understanding on this topic, both experimental work and analytical/kinetic Monte Carlo simulations are included in this thesis.

Chapter 2 outlines the basic physics of intrinsic organic semiconductors, especially in the role of the energetically broadened DOS and traps on charge transport. Traps generally have a detrimental effect on the performance of semiconductor devices. In Paper II we proposed a simple model to diagnose both the presence and the filling of traps and in Paper VIII we investigated the existence of a general rule for the trap distribution in organic semiconductors.

Chapter 3 discusses doping effects in organic semiconductors such as dopant impact on the DOS, mobility and doping efficiency, c.f. Paper I and II, and on Seebeck coefficient via different hopping model, c.f. Paper IV. In particular, the empirical universal power law is reproduced by the theoretical model in Paper IV. Also, the doping processing method has significant impact on thermoelectric properties as investigated in Paper VI.

Chapter 4 explores DOS engineering for tuning the thermopower and addresses the morphological influences on that in two-material system. In Paper III we illustrated the concept of DOS engineering and confirmed it for p-type OSCs, both experimentally and theoretically. The methodology was further successfully used for n-type materials in Paper V. The morphological influence was investigated in Paper VII. 


\section{Basic physics of conjugated polymers}

\subsection{Energy levels}

Organic semiconductors, especially conjugated polymers (CPs), have been studied intensively as promising active material in organic electronic devices like (organic) solar cells, lightemitting diodes, field-effect transistors and so on. ${ }^{47-49}$ Compared with their inorganic compound counterparts, one typical difference is that conjugated polymers consist of longchain molecules with a series of alternating single and double bonds between the carbon atoms. This kind of conjugated bonds result from so-called $\mathrm{sp} 2$ hybridized states. ${ }^{50}$ Whereas the $\mathrm{sp} 2$ hybridized orbitals are involved in the covalent bonding between $\mathrm{C}-\mathrm{C}$ and $\mathrm{C}-\mathrm{H}$ atoms, the remaining carbon $2 \mathrm{pz}$ orbital can offer its wavefunction to overlap with its neighboring $\mathrm{C}$ atom and form so-called hybrid $\pi$ (bonding) and pi* (antibonding) orbitals. The $\pi$-and pi* bonds support states that are shared along the polymer chain, that is delocalized states that are responsible for the charge transport.

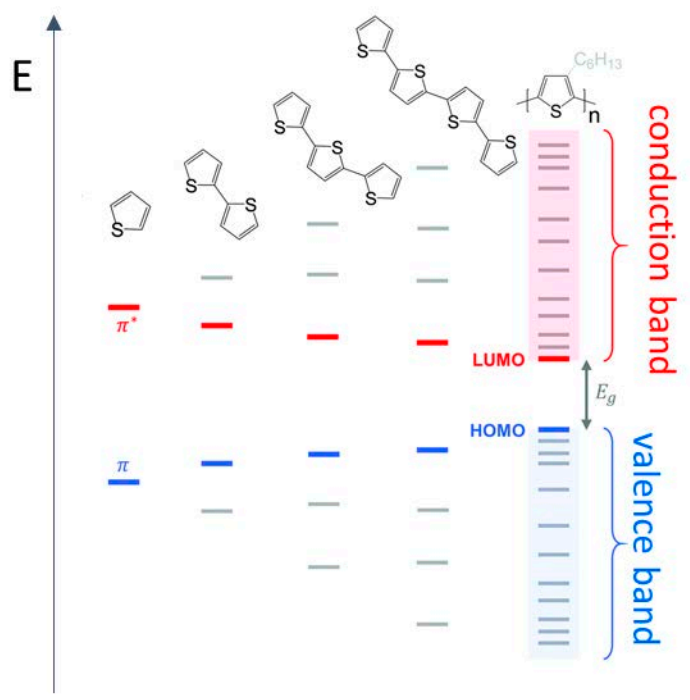

Figure 2.1 Evolution of the HOMO and LUMO levels as well as bandgap $E_{g}$ with increasing number of thiophene repeat units, resulting in valence and conduction bands for polythiophene. Adapted with permission from Ref. ${ }^{4}$.

The highest occupied molecular orbital (HOMO) and the lowest unoccupied molecular orbital (LUMO) are derived from the occupied $\pi$-bonding orbitals and the unoccupied $\pi^{*}$-anti-bonding orbitals, respectively. ${ }^{51}$ With increasing the length of the polymer chain, the bandgap between HOMO and LUMO becomes smaller as shown in Figure 2.1.

HOMO and LUMO energy levels determine many opto-and electrical-physics properties. For instance, the electron excitation process in organic solar cells, ${ }^{52}$ the doping effect in organic 
thermoelectics, ${ }^{53}$ and so on. Usually, the HOMO and LUMO energy level can be extracted from oxidation/reduction potentials, which can be obtained with cyclic voltammetry (CV) measurements. ${ }^{54}$ Ultraviolet photoelectron spectroscopy (UPS) is popular to characterize the electronic structure of organic materials. ${ }^{55}$ It can identify the ionization potential (IP), i.e. the energy required for moving an electron from a neutral polymer chain to the vacuum energy level (defined as $E=0 \mathrm{eV}$ ), and the electron affinity (EA), i.e. the energy obtained when adding an electron to a neutral polymer chain from the vacuum energy level. According to Koopmans' theorem, IP $(E A)=-H O M O(L U M O)$ only when the binding energy is equal to the orbital energy eigenvalue with the opposite site, i.e. without electronic and nuclear relaxation process. $^{56}$

\subsection{Density of states}

In condensed matter physics, the density of states (DOS) describes the number of states per unit volume that is available for charges to occupy at each energy. ${ }^{57}$ Conjugated polymers have long chains with alternating single and double bonds between carbon atoms, and typically the long backbone is not straight but twisted into 'any' possible conformation like the one shown in Figure 2.2. The twisted segments in the chain weaken the overlap of wavefunction between conjugated bonds, causing localized states with different energies due to the different conjugation lengths, different local environments etc. ${ }^{58-60}$ The density of states is used to describe those localized states, distributed in energy in conjugated polymer films containing a large number of polymer chains. ${ }^{57}$

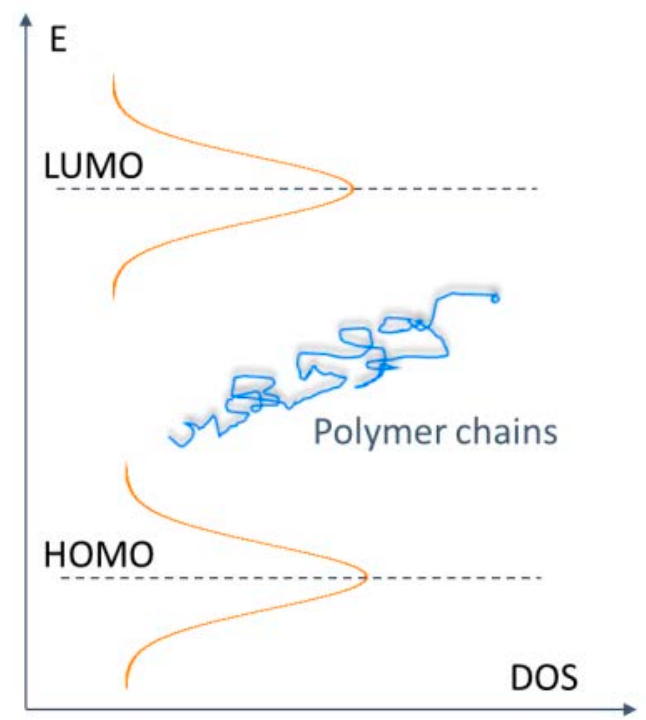

Figure 2.2 Illustration of Gaussian-shaped DOS for HOMO and LUMO levels related to the disordered structures of conjugated polymers. 
In some publications, mostly those dealing with organic field effect transistors, the shape of the DOS distribution of the disordered organic semiconductor (OSCs) is assumed to be an exponential, as e.g. used by Vissenberg and Matters. ${ }^{61}$

$$
g(E)=\frac{N}{k_{B} T_{0}} \exp \left(-\frac{E}{k_{B} T_{0}}\right)
$$

Here $N$ is the total DOS, and $T_{0}$ is a parameter that indicates the width of the exponential distribution.

Bässler et al. proposed a Gaussian shape of the DOS to characterize the distribution of localized states ${ }^{57}$ the expression is:

$$
g(E)=\frac{N}{\sqrt{2 \pi} \sigma_{D O S}} \exp \left(-\frac{\left(E-E_{i}\right)^{2}}{2 \sigma_{D O S}^{2}}\right)
$$

characterized by the energetical disorder $\sigma_{D O S}$ and $E_{i}$ the central energy of the HOMO or LUMO. Many researchers worked on the influence of the DOS shape on the electronic properties of organic semiconductors using simulations and experiments, and found that a Gaussian shape of DOS typically gives a reasonable description of experiments in bulk OSCs as used in diodes and solar cells. ${ }^{62-68}$ Therefore, we will take a Gaussian DOS as starting point to describe charge transport in disordered organic semiconductors in our work. 


\subsection{Charge transport in OSCs}

\subsubsection{Hopping model}

In conjugated polymers with a disordered nature as discussed above, one typically describes charge transport as thermally activated tunneling or 'hopping' between localized sites. ${ }^{57}$ The hopping rate from an occupied site $i$ to an empty site $j$ can be described by the Miller-Abrahams expression $^{69}$

$$
v_{i f}= \begin{cases}v_{0} \exp \left(-2 \frac{r_{i j}}{\alpha}\right) \exp \left(-2 \frac{E_{j}-E_{i}}{k_{B} T}\right), & \text { if } \Delta E>0 \\ v_{0} \exp \left(-2 \frac{r_{i j}}{\alpha}\right), & \text { if } \Delta E \leq 0\end{cases}
$$

where $v_{0}$ is the attempt-to-hop frequency, $r_{i j}$ is the distance between site $i$ and $j, \alpha$ is the localization length of charge carriers in localized states, $E_{i(j)}$ is the energy of the site and $\Delta E=$ $E_{j}-E_{i}$.

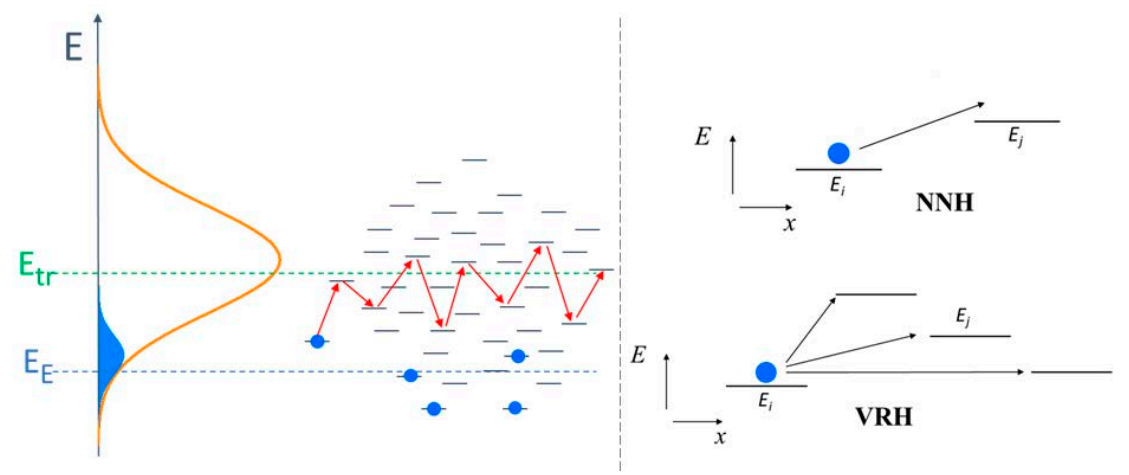

Figure 2.3 Schematic behavior of charge transport within a Gaussian-shaped DOS (left) and the NNH and VRH model (right).

In the case of $\Delta E>0\left(E_{j}>E_{i}\right)$, the charge has to decide whether to jump from site $i$ to a nearest neighbor site $\mathrm{j}$, a next -nearest neighbor site or a site even further away. What happens is decided not only by the spatial factor $\Delta x\left(r_{i j}\right)$, also by the energy difference $\Delta E$. This allows to divide hopping models into two different cases: nearest neighbor hopping $(\mathrm{NNH})$ and variable range hopping (VRH) models as shown in Figure 2.3. The NNH model describes the charge carrier motion as hopping to its nearest neighbor sites and this process is only assisted by energy. ${ }^{70,71}$ In contrast, in VRH the site the charge carrier hops to is determined by a tradeoff between the 'cost' of hopping in real space and hopping in energy space. ${ }^{22,72}$ It should be noted that NNH is the high-temperature limit of VRH. ${ }^{71}$ In other words, at sufficiently low temperatures hopping systems will show VRH behavior. 


\subsubsection{Fermi energy and equilibrium energy}

The DOS describes all possible states that can be occupied by charge carriers. The distribution of occupied states is calculated by taking the product of the DOS with Fermi-Dirac distribution

$$
f(E)=\frac{1}{e^{\left(E-E_{F}\right) / k_{B} T}+1}
$$

where $E_{F}$ is Fermi energy. In an exponential DOS, most carriers occupy energies in the vicinity of $E_{F}$ (assuming $\mathrm{T}<T_{0}$ ). ${ }^{22}$ However, in a Gaussian DOS at low carrier concentration, most carriers in thermal equilibrium are not distributed around the Fermi energy. On the contrary, those carriers are located around the so-called equilibration energy $\left(E_{\infty}\right){ }^{73}$

$$
E_{\infty}=-\frac{\sigma_{D O S^{2}}}{k_{B} T}
$$

The equilibration energy is, at constant temperature and for a constant $\sigma_{D O S}$, independent of carrier concentration while the Fermi energy is dependent on the carrier concentration. The trends in $E_{F}$ and $E_{\infty}$ with carrier concentration are shown in Figure 2.4. At a given temperature the curves for $E_{F}$ and $E_{\infty}$ intersect, which means that $E_{\infty}$ is no longer a good measure of the typical charge carrier energy and that the system is no longer in the Boltzmann limit. ${ }^{22}$

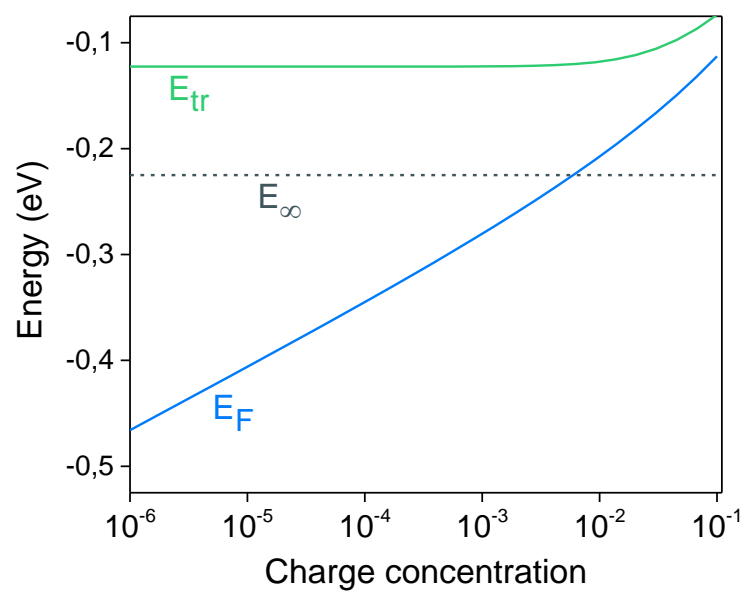

Figure 2.4 The positions of Fermi energy $\left(E_{F}\right)$, equilibration energy $\left(E_{\infty}\right)$ and transport energy $\left(E_{t r}\right)$ varying with relative charge concentration for pristine OSCs. Calculation parameters in the VRH model (for further details see Chapter 3): inter-site distance $a_{N N}=1.8 \mathrm{~nm}$, Gaussian disorder $\sigma_{D O S}=75 \mathrm{meV}$, Temperature $T=300 \mathrm{~K}$, inverse localization length $\alpha=5 \mathrm{e} 8 \mathrm{~nm}^{-1}$, attempt-to-hop frequency $v_{0}=1 \mathrm{e}^{13} \mathrm{~s}^{-1}$. 


\subsubsection{Transport energy}

According to percolation theory, DC conductivity, which needs a connected path through the entire system, can be characterized by a typical hop. ${ }^{74}$ The typical hop describes carriers hopping from an initial site at a typical energy $E_{F}$ (beyond the Boltzmann limit) or $E_{\infty}$ (in the Boltzmann limit), to a final site at a particular energy level, ${ }^{22,71,75}$ the so-called transport energy $\left(E_{t r}\right)$ seen in Figure 2.5.

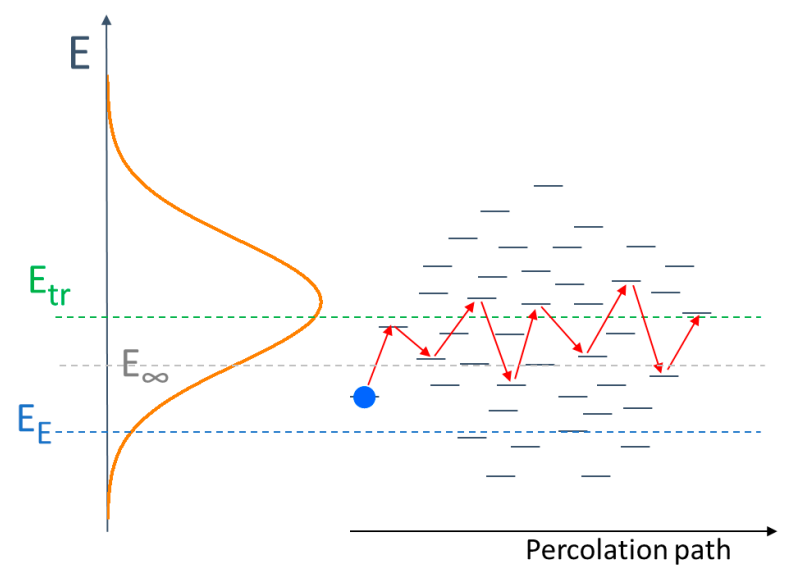

Figure 2.5 Illustration of the charge carrier transport energy in a Gaussian DOS to form a percolation path.

The transport energy can be calculated by optimizing the hopping probability under the percolation condition that each site in the percolating network is connected to a certain number (typically 2.8) of other sites. For a Gaussian DOS, $E_{t r}$ is situated slightly below the maximum of the DOS, ${ }^{57,76}$ that means that $E_{t r}$ for electrons (holes) is close to the LUMO (HOMO) energy level. $E_{t r}$ is essentially independent of carrier concentration up to very high concentrations as shown in Figure 2.6.
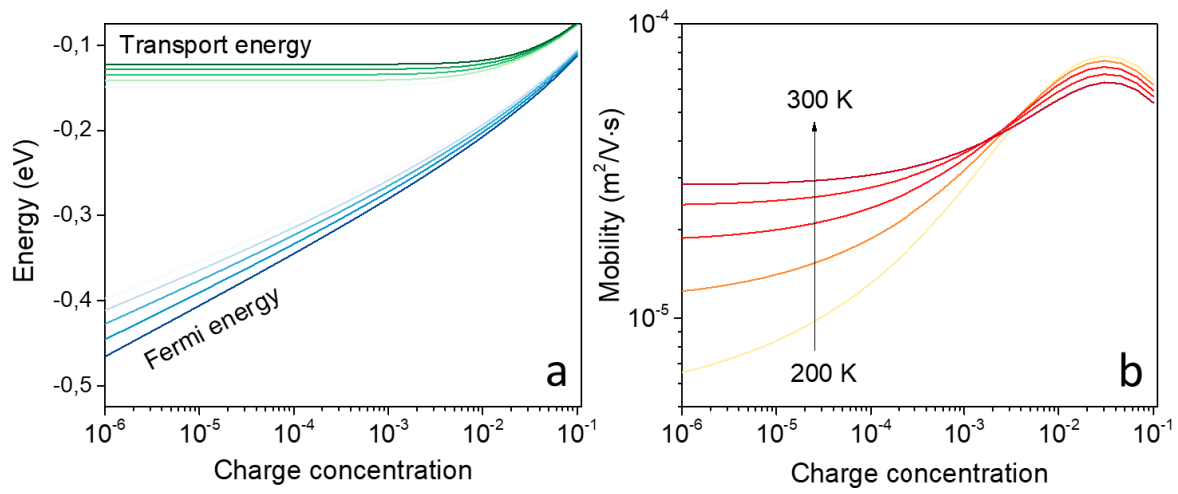
Figure 2.6 a. Fermi energy and transport energy, and b. mobility varying with relative charge concentration and temperature $(200 \mathrm{~K}$ to $300 \mathrm{~K}$ ) for pristine OSCs. Calculation parameters in the VRH model: inter-site distance $a_{N N}=1.8 \mathrm{~nm}$; Gaussian disorder $\sigma_{D o s}=75 \mathrm{meV}$; inverse localization length $\alpha=5 \mathrm{e} 8 \mathrm{~nm}^{-1}$; attempt-to-hop frequency $v_{0}=1 \mathrm{e}^{2} 3 \mathrm{~s}^{-1}$.

Summarizing, the Fermi energy (or equilibrium energy in a Gaussian DOS at low concentration) gives a prediction of where most carriers are situated, and the transport energy is a measure of the energy they have to hop to in order to contribute to the DC conductivity. These three energies mainly determine the charge transport in OSCs, in particular the physical properties of mobility, conductivity and Seebeck coefficient as will be discussed next. As we are eventually interested in the properties of highly conductive, i.e. strongly doped materials, we will in the following focus on systems in which the charge carrier concentration is non-negligible, i.e. where $E_{F}$ instead of $E_{\infty}$ should be used to characterize the transport.

\subsubsection{Mobility}

In semiconductors, the mobility characterizes the speed of charge carrier transport under influence of an electric field, i.e. $v=\mu F$. Above, we have shown that the transport is basically determined by the position of the Fermi energy and the transport energy. Specifically, the conductivity $\sigma$ becomes a Boltzmann factor of their difference:

$$
\sigma \propto \exp \left(\frac{E_{F}-E_{t r}}{T}\right)
$$

Here, $E_{F}$ is a function of carrier concentration, but usually not $E_{t r}$ as shown in Figure 2.6. The conductivity and mobility are related as $\sigma=e n \mu$ where $n$ is the charge carrier density. A typical example of the mobility versus carrier concentration for a VRH hopping system is shown in Figure 2.6.

In Figure 2.6 (a), for increasing but not too high charge density the gap between Fermi energy and transport energy obviously decreases due to the up-moving $E_{F}$ while $E_{t r}$ is almost constant. Thereby an increase in mobility is seen in Figure 2.6 (b). We note that $E_{F}-E_{t r}$ is almost constant at high charge concentrations, resulting in a conductivity that is largely independent on charge density. Mobility is calculated by $\mu=\sigma / e n$, and unsurprisingly the mobility decreases with higher charge concentration in this regime.

Besides the impact of carrier concentration on mobility, the mobility is strongly dependent on many parameters of which the applied field, the temperature, the typical inter-site distance, the energetical disorder and the attempt-to-hop frequency are the most important ones. ${ }^{57,61,70,77-79}$ For instance, Figure 2.6 shows the mobility dependence on carrier concentration for different temperatures, and the mobility gradually increases with higher temperature in hopping transport. 


\subsection{Traps}

Another complicating factor in many organic semiconductors are traps, for both electrons and holes. Many aspects of traps in organic semiconductor are still unclear, e.g. the origin of traps and their distribution in energy and position in CPs. Some researchers suggested the intrinsic defects caused by twists and kinks of polymer backbone, impurities remaining from solvents and synthesis, and contamination from processing environment, act as the cause of electron traps. ${ }^{58-60,80,81}$

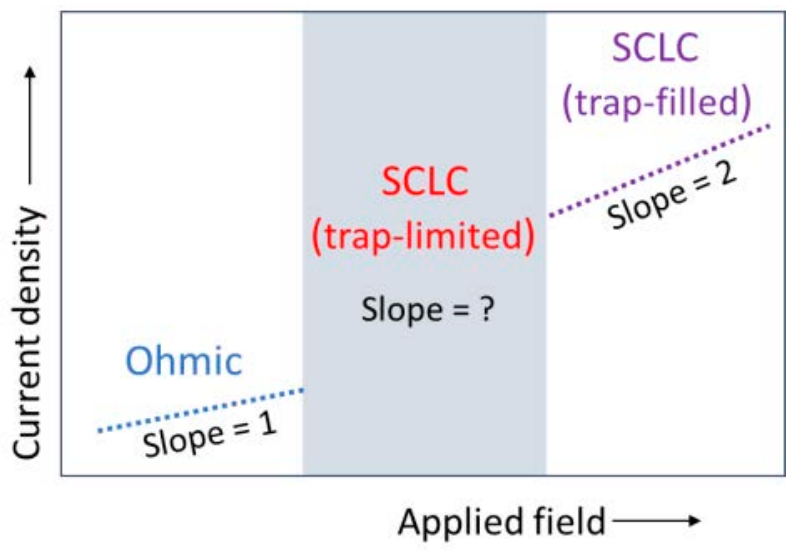

Figure 2.7 Illustration of typical regimes with slope values in current-voltage curves from space-charge-limited-current measurements.

During the last decade, space-charge-limited-current (SCLC) measurements have been established as a facile tool to determine the presence of traps in disordered organic semiconductors. ${ }^{82,83}$ In such cases, the current-voltage $(J-V)$ curve can be typically divided into three characteristic regimes as shown in Figure 2.7. With increasing applied field, the three regimes are the Ohmic regime (slope=1), the trap-limited SCLC regime (slope $>>2$ ), and the trap-filled SCLC regime (slope $=2$ or more) ${ }^{84}$

In case the traps are distributed exponentially with a characteristic temperature $T_{0}$, the $J-V$ curve can be characterized by the expression ${ }^{82}$

$$
J=N_{C} e \mu\left(\frac{\varepsilon_{0} \varepsilon_{r}}{e N_{t}}\right)^{r} \frac{V^{r+1}}{L^{2 r+1}} C(\mathrm{r})
$$

where $N_{C}$ is effective DOS, $N_{t}$ is the trap density; $r$ is $T_{0} / T ; C(\mathrm{r})=r^{r}(2 r+1)^{r+1}(r+$ $1)^{-2 r-1} ; \varepsilon_{0}$ and $\varepsilon_{r}$ are the absolute and relative dielectric constant.

Since charge transport in bulk organic semiconductors is usually described by hopping in a Gaussian DOS distribution, it would be probable to also describe the trap states by a Gaussian distribution instead of exponential one. Indeed, Nicolai et al. experimentally show that a Gaussian trap model well describes the trap distribution in three PPV derivatives, and leads to a more consistent description than an exponential distribution. ${ }^{85}$ In our work we use the same 
Gaussian model to express the trap distribution as Ref. ${ }^{80}$. In such a Gaussian distribution of traps, the slope of $J$ - $V$ curves is typically related to the density and energetic distribution of the traps. Using the slope of $J$ - $V$ curves on a wide range of conjugated polymers, Nicolai et al. concluded the existence of a universal electron trap, centered at an energy of $\sim 3.6 \mathrm{eV}$ with a density of $\sim 3 \times 10^{23}$ traps per $\mathrm{m}^{3}$. Therefore, they argued that the electron traps have a common origin likely related to water or oxygen due to its redox potential sitting close to this energy. ${ }^{80}$

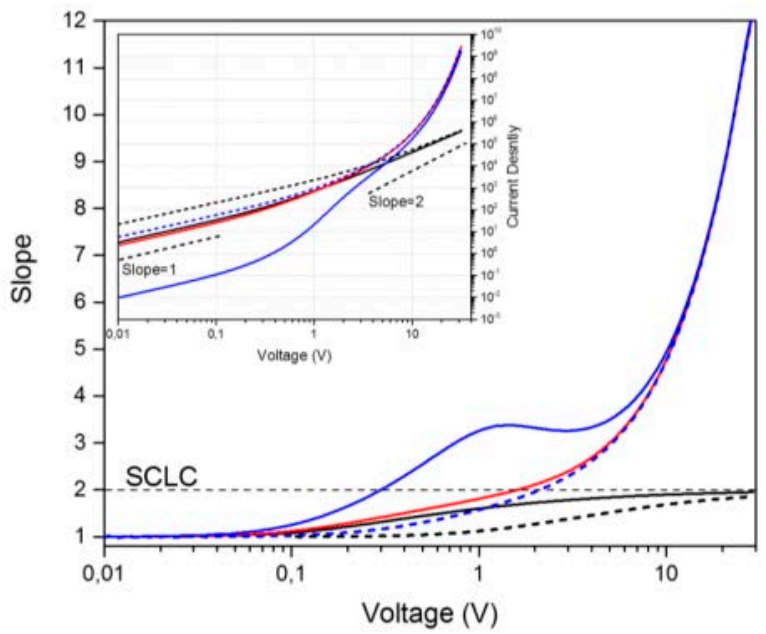

Figure 2.8 Calculated slope vs. bias curves from the drift-diffusion model with constant mobility (solid black line), Gaussian disorder + no trap (solid red line), Gaussian disorder + trap (solid blue line), Gaussian disorder + trap +doping (dashed blue line) and constant mobility + doping (dashed black line). The insert shows the corresponding $J-V$ curves. The parameters used are: $a_{N N}=1.8 \mathrm{~nm}, v_{0}=1 \mathrm{e}^{1} 1 \mathrm{~s}^{-1}$, and $\sigma_{D O S}=80 \mathrm{meV}$, corresponding to $\mu_{0}=6 \mathrm{e}-8 \mathrm{~m}^{2} / \mathrm{Vs}$, $N_{t}=1 \mathrm{e} 23 \mathrm{~m}^{-3}, E_{t}=0.35 \mathrm{eV}, N_{\text {dope }}=1 \mathrm{e} 23 \mathrm{~m}^{-3}$, and $T=300 \mathrm{~K}$. Adapted with permission from Paper II.

In order to quantitatively and qualitatively investigate the trap behavior, we developed a novel and simple method to more easily observe the particular trapping and trap filling process. The method is based on the inspection of the logarithmic slope of unipolar J-V curves, following: ${ }^{86}$

$$
\text { slope }=\frac{d(\log J)}{d(\log V)}
$$

as illustrated in Figure 2.8. Interpretation of the obtained traces was facilitated by using a 1D drift-diffusion model that accounts for Gaussian disorder through a density- and fielddependent mobility, trapping in an additional Gaussian trap level (assumed to be of equal width as the HOMO DOS). We calculated the $J-V$ curves and slopes with/without traps as shown in Figure 2.8. In the presence of traps (solid blue curve), a peak occurs at the transition between trap-limited rand trap-filled regimes, in contrast to the smooth upswing curve that is observed for trap-free J-V case (red curve). In Paper II and VIII we demonstrate that our slope-method 
is quite easily applicable to identify traps and can be used to analyze trap formation and filling behavior. In combination with the mentioned drift-diffusion model, it can also be used to quantitatively determine trap energies and densities.

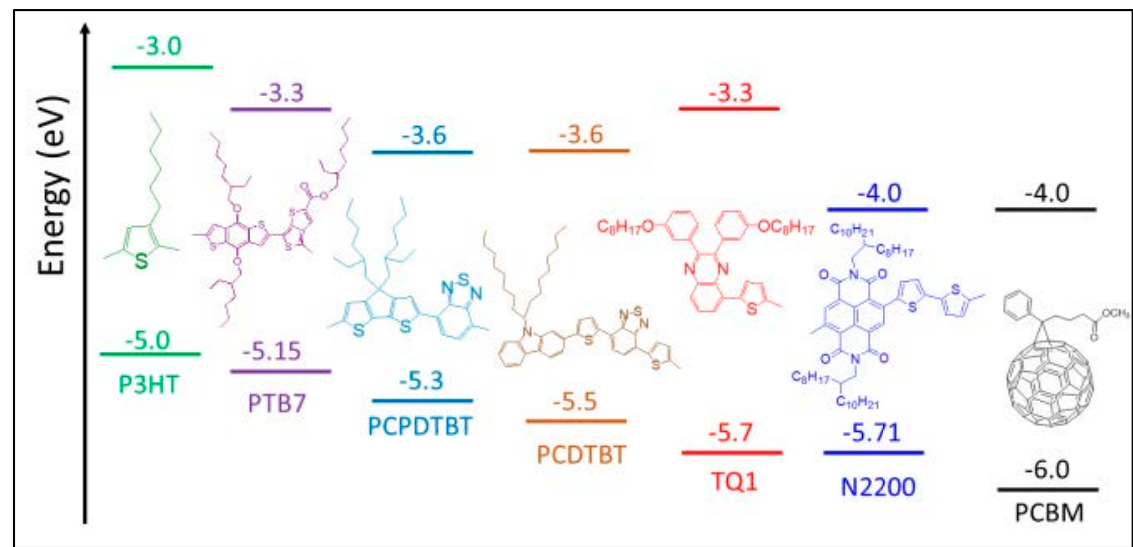

Figure 2.9 Chemical structure and HOMO and LUMO energy levels of the materials used for determining hole and electron trap energies and concentrations.

Coming back to the origin of traps, one can wonder if it does have a common or universal origin for electrons or holes? Nicolai et al. investigated the J-V characteristic based on electron-only devices and suggested a common trap level, centered at an energy of about $3.6 \mathrm{eV}$ for electron trap, most likely related to hydrated oxygen complexes. Using the refined method described above, we derived both electron and hope trap energies, and the results are shown in Figure 2.10 for many organic semiconductors with various energy levels seen in Figure 2.9. In stark contrast to the findings in Ref. ${ }^{80}$, we find the trap energies are not constant, but rather are located at a roughly constant difference of 0.3-0.4 eV above (below) the HOMO (LUMO). 


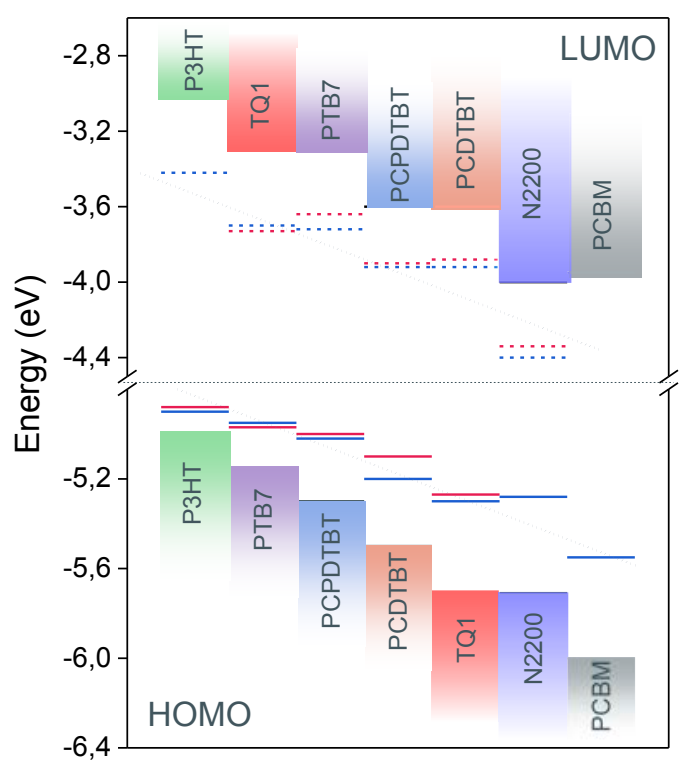

Figure 2.10 Hole (solid lines) and electron (dashed lines) trap energies in relation to HOMO (shaded area on bottom) and LUMO (top) energy levels for the investigated range of organic materials. Trap energies determined by slope- and $J$ - $V$-fitting are shown as red and blue lines, respectively. The thin black dotted lines are guides to the eye. 


\section{Doping}

Previously we mentioned most of organic semiconductors exhibit a low intrinsic charge carrier density $n_{\text {int }}$ due to energetic disorder and the large bandgap, restricting application of OSC in electronic devices. Fortunately, much research indicates that doping can significantly improve the performance of OSCs and extend the application in various fields like OPV, LED, OFET, OTE and so on. ${ }^{87-90}$ At the same time doping has some drawbacks. ${ }^{91}$ In this chapter we comprehensively explore the nature of doping.

\subsection{Doping types}

The charge carrier concentration in intrinsic disordered organic semiconductors is typically low. Hence, also their conductivity is low, which is undesired for many applications, including thermoelectrics. Doping is effective way to increase electrical conductivity. According to the origin of the dopant, doping can typically be classified in one the four types discussed below. To facilitate comparison, the selected examples from literature focus on the semiconductor P3HT, which is the field's testbed system of choice. ${ }^{90,92-100}$

\subsubsection{Chemical doping}

Chemical doping is also called acid-base doping, and its dopant normally is a type of inorganic compound like $\mathrm{FeCl}_{3}, \mathrm{I}_{2}, \mathrm{NOPF}_{6}$ and so on. More dopants and their structures can be seen in section 3.4. The doping effect originates from the transfer of a cation or anion to the backbone of the host material when the dopant is dissolved in solution as illustrated in Figure 3.1.

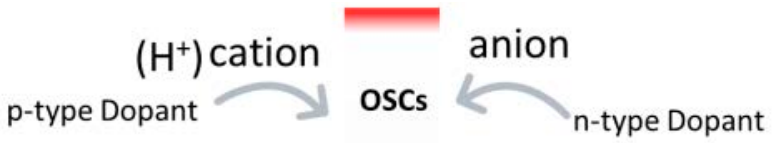

Figure 3.1 Illustration of the principle of chemical doping, referring anion (cation) transfer.

For instance, Hong et al. achieved a high electrical hole conductivity over $2.5 \times 10^{4} \mathrm{~S} / \mathrm{m}$ from a ferric chloride $\left(\mathrm{FeCl}_{3}\right)$ doped P3HT film and a power factor of up to $35 \mu \mathrm{W} / \mathrm{m} \cdot \mathrm{K}^{2}{ }^{90}$ Xuan et al. studied the effect of $\mathrm{NOPF}_{6}$ concentration on the thermoelectric properties of P3HT and obtained a high p-type conductivity of $\sim 1000 \mathrm{~S} / \mathrm{m} .{ }^{92}$ Sakiyama et al. used $\mathrm{CsCO}_{3}$ as n-type dopant, achieving a conductivity up to $10^{-4} \mathrm{~S} / \mathrm{m}$ in MEH-PPV film adding $2 \mathrm{wt} . \% \mathrm{CsCO}_{3}$ while the conductivity $\sim 10^{-9} \mathrm{~S} / \mathrm{m}$ in undoped MEH-PPV film. ${ }^{101}$ Despite chemical doping achieving high conductivity, the stability of the electronic and thermoelectric properties like Seebeck coefficient is a big problem, limiting the use of this doping method. 


\subsubsection{Molecular doping}

Molecular doping involves the transfer of one or more electrons between the HOMO and LUMO energy levels of the host and guest (dopant) materials. If the electron transfers from HOMO of the host material to the LUMO of the dopant, it is so-called p-type doping, vice versa it is n-type doping. This type of doping mechanism and the desired energetic alignment is illustrated in Figure 3.2.

Normally, in this type of doping, the dopant is an organic molecule like $\mathrm{F}_{4} \mathrm{TCNQ}$, TDAE, NDMBI and so on. More dopants and their structures are shown in section 3.4. For example, $\mathrm{F}_{4} \mathrm{TCNQ}$ with a LUMO of $-5.24 \mathrm{eV},{ }^{102}$ is a good p-type dopant for P3HT (HOMO, $\left.-5.0 \mathrm{eV}\right){ }^{103}$ and there are many works on $\mathrm{F}_{4} \mathrm{TCNQ}$-doped P3HT being investigated for organic thermoelectrics. Sun et al. achieved an electrical conductivity $\sim 4 \times 10^{-2} \mathrm{~S} / \mathrm{m}$ and power factor around $6 \times 10^{-3} \mu \mathrm{W} / \mathrm{m} \cdot \mathrm{K}^{2}$ by adding $2 \mathrm{wt} . \% \mathrm{~F}_{4} \mathrm{TCNQ}$ into the P3HT casting solution. ${ }^{104}$ For ntype doping, Wei et al. proposed a novel organic molecule N-DMBI. When using this dopant, the conductivity of a PCBM film increased more than 4 orders of magnitude compared to the undoped case, reaching a value of $\sim 0.19 \mathrm{~S} / \mathrm{m}^{43}$

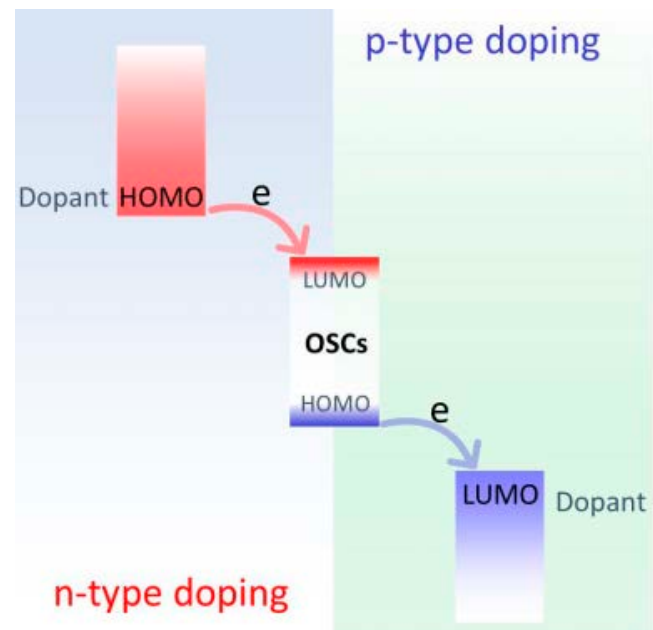

Figure 3.2 Illustration of the principle of molecular doping, referring electron transfer.

We note that one drawback of molecular doping is the strict requirement of having a dopant with low-lying HOMO or high-lying LUMO to match the host materials' LUMO or HOMO, respectively. Nonetheless, due to the stability of some of these dopants, especially for the ptype materials, molecular doping can dramatically enhance the overall thermoelectric performance of organic materials in ambient air. As such, it still is considered as a very promising method in organic thermoelectrics. 


\subsubsection{Secondary doping (Morphology effect)}

Unlike the above two doping cases, secondary doping does not need a dopant medium that gives rise to electron transfer. The 'doping effect' is typically an increased conductivity that results from the morphological improvement of the material system using additive solvents. ${ }^{105}$ The mobile charges still must be induced by other means, e.g. as described above. There are many reports of secondary doping in the case of PEDOT:PSS. ${ }^{106-109}$ For instance, Ouyang et al. demonstrated that the conductivity of PEDOT:PSS increased from $30 \mathrm{~S} / \mathrm{m}$ up to $7 \times 10^{4} \mathrm{~S} / \mathrm{m}$ after adding polar organic solvents such as EG and DMSO and thermal treatment. ${ }^{105}$ Patel et al. also found the morphology heavily impacting the thermoelectric properties in the doped semiconducting polymer PBTTT. ${ }^{36}$ In this case the 'secondary dopant' is the substrate treatment performed before polymer deposition.

For PEDOT:PSS, the significant improvement in conductivity is believed to result from changes in the phase segregation between PEDOT and PSS. The phase segregation results in removing some of the PSS from the PEDOT:PSS films and changing the conformation of PEDOT chains from a coil structure to an extended-coil or linear structure. That then results in enhance charge transport along the PEDOT chains. Although processing-induced secondary doping and morphology effects have been widely studied the precise mechanisms are still under heavy debate. ${ }^{105}$

We note that the additive DIO effect is likely to that of solvents in PEDOT:PSS case, where in the sense that DIO affects the phase separation in a blend system, which in turn affects the thermoelectric properties, will be discussed in Chapter 4.

\subsubsection{Electrochemical doping}

Electrochemical doping is partially like chemical doping, and its dopant is normally also a type of inorganic/organic compound containing a mobile ion like $\mathrm{K}^{+}, \mathrm{Li}^{+}, \mathrm{I}^{-}$and so on in solution or electrolyte. ${ }^{110}$ The difference is that in chemical doping the doping effect originates from carrier injection into the OSC from an electrode while the balancing redox reaction causes the formation of cations or anions. Electrochemical doping is very popular in the field of lightemitting devices and (ionic) transistors. ${ }^{111-114}$ For instance, the first application of electrochemical doping in light-emitting device (LEC) was discovered by Pei et al. ${ }^{113}$ In LECs, applying a voltage across the active material leads to a simultaneous $\mathrm{p}$ - and $\mathrm{n}$-doping in the vicinity of the two electrodes, respectively, ${ }^{111}$ while the mobile ions keep the electrochemical equilibrium. Since such devices strongly depend on mobile ions, this limits the possible choices of the matrix or active layer, which has to be ion-conducting. 


\subsection{Doping efficiency}

In principle, for systems like P3HT:F4TCNQ, there are two typical models to describe the charge transfer between the host material and the dopant, integer charge transfer (ICT) and hybrid charge transfer complex (CTC). ${ }^{15,116}$ Since F4TNCQ has a lower-lying LUMO than P3HT's HOMO, it is energetically favorable for every $\mathrm{F}_{4} \mathrm{TCNQ}$ dopant to undergo integer charge transfer between F4TNCQ's LUMO and P3HT's HOMO. Indeed, Wang et al. found a charge transfer efficiency approaching unity in this system. ${ }^{117}$ This was also found by Pingel et al. who, however, indicated that only about $5 \%$ of these charge carrier pairs actually dissociate and contribute a free hole for electrical conduction. ${ }^{116}$ In contrary, Aziz et al. proposed the formation of a CTC whose HOMO and LUMO derived from hybridization of the neutral P3HT HOMO and F4TNCQ's LUMO. ${ }^{118}$ In our work, we only focus on the ICT model as this turns out to give an accurate description of our findings in Paper I, II, IV and V.

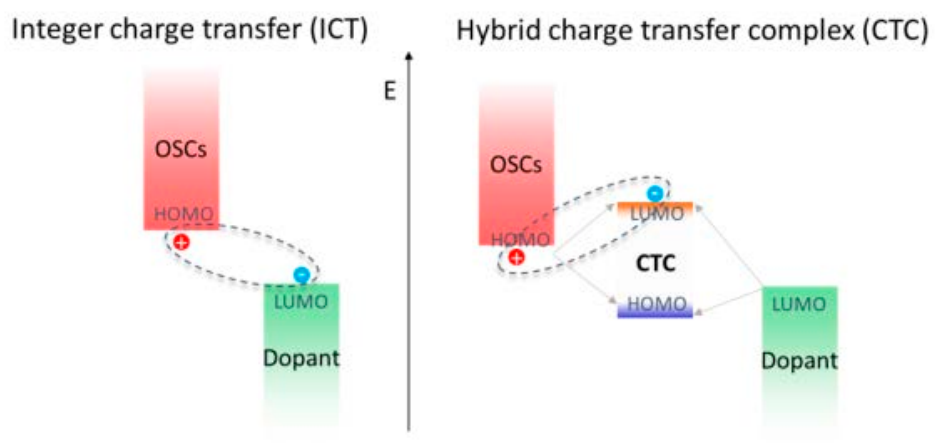

Figure 3.3 Illustration of the charge transfer for integer charge transfer (ICT) model and hybrid charge transfer complex (CTC) model.

To investigate the reason for the difference between almost $100 \%$ charge transfer efficiency and the low fraction of mobile charges as e.g. found by Pingel, ${ }^{116,117}$ we studied the spatial distribution of the charge carriers with respect to the dopant counterions. For this, we used a kinetic Monte Carlo tool (see section 1.8) and made the assumption that every dopant molecule produces a charge carrier (integer charge transfer, see above), leaving behind an ionized dopant that acts as a Coulomb trap for the charge. In the simulation, we distinguish three situations. Either, a charge sits at the position of a dopant ion (trapped charge), it sits one site away from a dopant (CT charge), or it sits elsewhere (free charge). Figure 3.4 shows that the free fraction decreases steeply with a concentration above $10^{-2}$, for the simple statistical reason that there are hardly any sites left in the system that are not a dopant or a CT site. At lower concentrations, the fraction of free charges also decreases with increasing dopant concentration. We attribute this effect to the fact that with increasing concentration the time between escape from one Coulomb trap and capture by another also decreases. Although the time spent at an individual 
dopant site during a specific event goes down with doping concentration, the total time spent at dopant sites in general goes up.
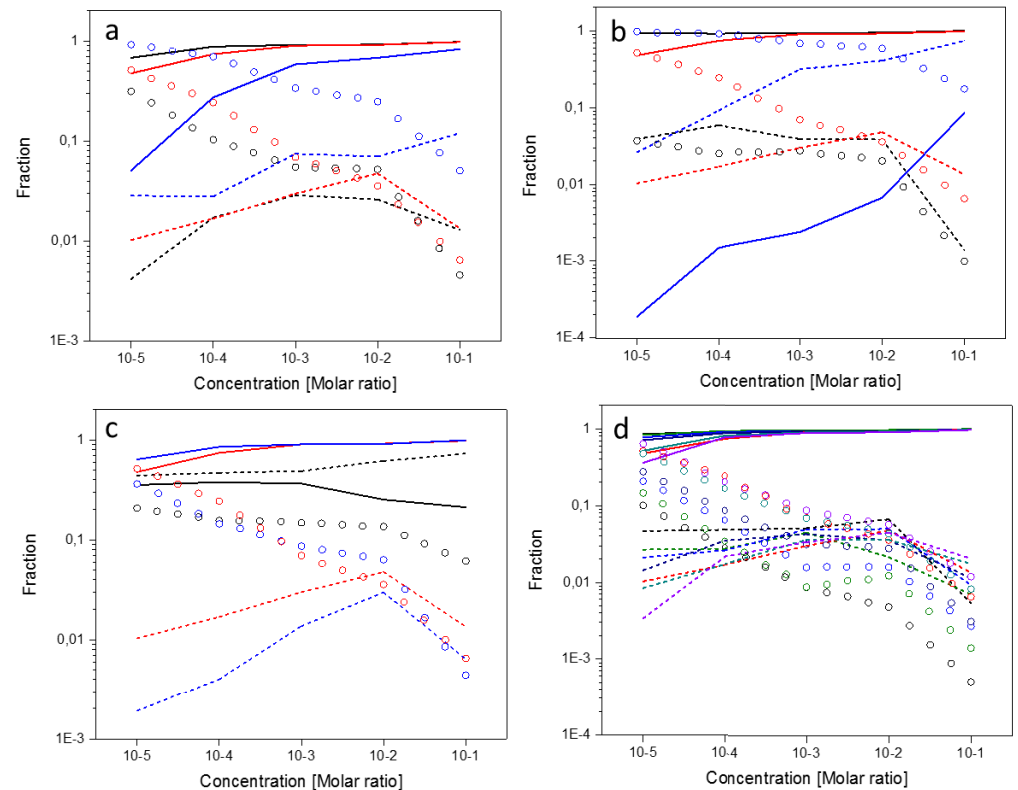

Figure 3.4 Fraction of different carrier charges (trap charges (solid line), CT charges (dashed lines) and free charges (dots)), with various doping concentrations from $\mathrm{kMC}$ for different parameters: a. energetic disorder: $\sigma_{D O S}=0.05 \mathrm{eV}$ (black), $0.075 \mathrm{eV}$ (red) and $0.1 \mathrm{eV}$ (blue); b. energy level difference: $\Delta \mathrm{E}=-0.2 \mathrm{eV}$ (black), $0 \mathrm{eV}$ (red) and $0.3 \mathrm{eV}$ (blue); c. inter-site distance $a_{N N}=1.0 \mathrm{~nm}$ (black), $1.8 \mathrm{~nm}$ (red), $3.0 \mathrm{~nm}$ (blue); d. temperature: $T=200 \mathrm{~K}, 225 \mathrm{~K}, 250$ $\mathrm{K}, 275 \mathrm{~K}, 300 \mathrm{~K}, 320 \mathrm{~K}, 340 \mathrm{~K}$. Adapted with permission from Paper I.

We loosely consider the doping efficiency as the fraction of free charges, that may or may not also include the fraction of CT charges. Regardless of the precise definition, the doping efficiency is eventually below unity in a large fraction of the parameter space like energetic disorder, energy level difference, inter-site distance and temperature. The physical reason is simply that the ionized dopants Coulombically bind the 'mobile' countercharges. Further details are discussed in Paper $\mathbf{I}$. 


\subsection{Molecular doping methods}

\subsubsection{Bulk doping}

In conventional solution processing, the doped film is deposited from a common solution containing both organic semiconductor and dopant, which we shall refer to as bulk doping. This is illustrated in Figure 3.5; a very typical application studied in this thesis is the addition of $\mathrm{F}_{4} \mathrm{TCNQ}$ into a P3HT solution for organic thermoelectrics. A major problem with the bulk doping method is that the large amount of $\mathrm{F}_{4}$ TCNQ negatively affects the morphology of the P3HT film and leads to F4TCNQ aggregation, preventing one to achieve higher conductivity.
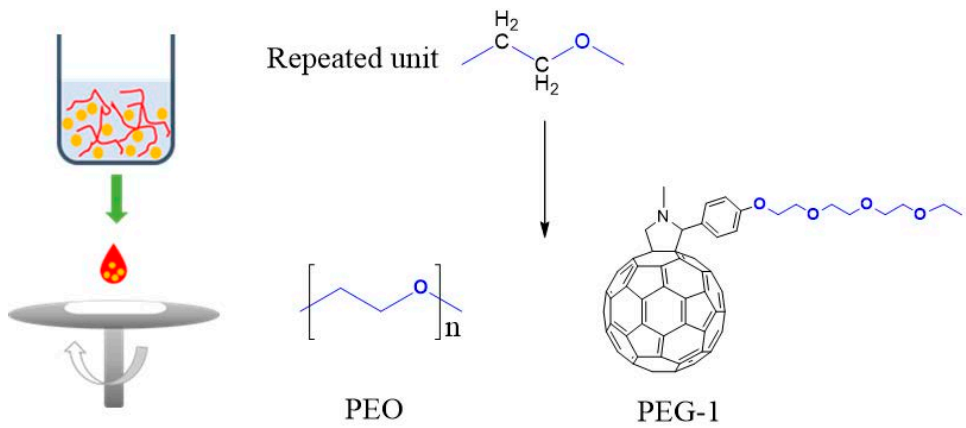

Figure 3.5 Schematic of the bulk doping process to fabricate devices (left) and structures of PEO and TEG-1 containing ethylene oxide unit (right).

Kiefer et al. investigated the compatibility of $\mathrm{F}_{4} \mathrm{TCNQ}$ with $\mathrm{P} 3 \mathrm{HT}$ in solution and suggested the addition of semi-crystalline poly(ethylene oxide) (PEO) to increase the solution stability. With 62 wt.\% PEO (in total weight) and 20 mol.\% F 4 TCNQ in PEO+F4TCNQ+P3HT blends' solution, the doped P3HT film yielded an electrical conductivity $\sim 30 \mathrm{~S} / \mathrm{m}$ and a power factor $\sim 0.1 \mu \mathrm{W} / \mathrm{m} \cdot \mathrm{K}^{2}$, whereas the conductivity less than $10^{-2} \mathrm{~S} / \mathrm{m}$ for the same amount of F4TCNQ doped into P3HT without PEO. ${ }^{119}$

We note that structures containing the repeat unit (- $\left.\mathrm{CH}_{2}-\mathrm{CH}_{2}-\mathrm{O}-\right)$ like PEO and PEG-1 have a strong solvent-polar effect, enhancing the miscibility of the host/dopant system. Liu et al. brought in such structure as sidechain for PCBM and N2200, achieving an electrical conductivity $\sim 205 \mathrm{~S} / \mathrm{m}$ (100 times higher) and $\sim 17 \mathrm{~S} / \mathrm{m}$ (200 times higher) in comparation with the pristine counterpart doped by N-DMBI, respectively. ${ }^{40,120}$ Thus, in bulk doping this method can improve the compatibility in solution, allowing one to use a larger fraction of the dopant like $\mathrm{F}_{4} \mathrm{TCNQ}$ and N-DMBI. However, the limited enhancement of the electrical conductivity still restricts the overall thermoelectric performance. 


\subsubsection{Sequential doping}

Unlike bulk doping, sequential doping achieves high electrical conductivity, while preserving the film morphology by spin-coating the dopant solution on a previously cast semiconductor film. ${ }^{99}$ Figure 3.6 illustrates how sequential doping can be used to fabricate an F4TCNQ doped P3HT film.

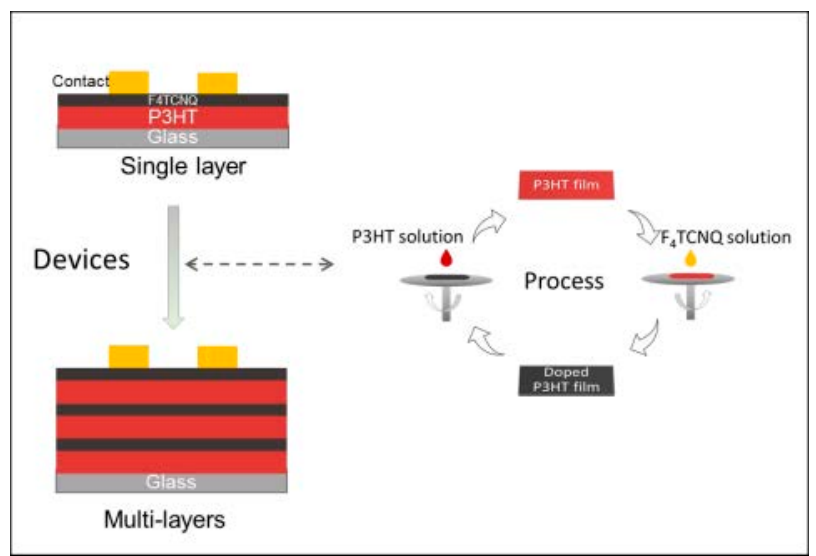

Figure 3.6 Schematic of the sample structure and of the sequential doping process to fabricate single and multilayer devices.

Scholes et al. indicated an electrical conductivity as high as $550 \mathrm{~S} / \mathrm{m}$ achieved by $\mathrm{F}_{4} \mathrm{TCNQ}$ doped P3HT film using sequential doping. ${ }^{91}$ Hamidi-Sakr et al. investigated anisotropic charge transport in ( $\left.\mathrm{F}_{4} \mathrm{TCNQ}\right)$ sequentially doped highly oriented P3HT and PBTTT films, including measurements of the Seebeck coefficient. ${ }^{121}$ However, there is still only limited experimental work on thermoelectric properties using sequential doping for achieving high conductivity.

We prepared single P3HT films with a range of thicknesses from $25 \mathrm{~nm}$ to $500 \mathrm{~nm}$ and used sequential doping by $\mathrm{F}_{4} \mathrm{TCNQ}$ to investigate the electrical conductivity, Seebeck coefficient and power factor. Using this surface doping method, we arrive at electrical conductivities over $400 \mathrm{~S} / \mathrm{m}$ and at a power factor $\sim 8 \mu \mathrm{W} / \mathrm{m} \cdot \mathrm{K}^{-2}$ for thin $(<100 \mathrm{~nm})$ films. ${ }^{31}$ For contrast, results for bulk doping at different dopant concentrations are shown in Figure 3.7b. Unsurprisingly, the bulk doped samples give much lower conductivities, and associated with that, larger thermopowers that combine into low PFs of $10^{-3}-3 \times 10^{-2} \mu \mathrm{W} / \mathrm{m} \cdot \mathrm{K}^{-2}$. These numbers are consistent with PFs around $10^{-3}-10^{-1} \mu \mathrm{W} / \mathrm{m} \cdot \mathrm{K}^{-2}$ that were previously reported for the same material combination using bulk doping. ${ }^{104}$ 

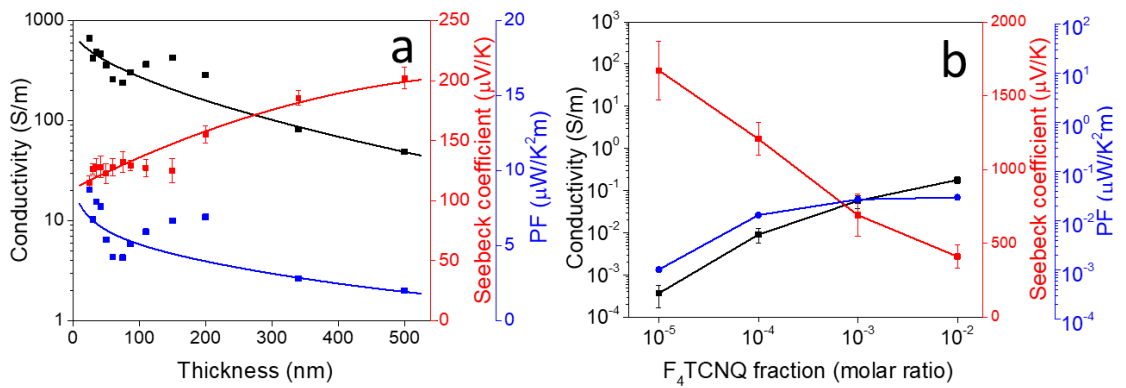

Figure 3.7 a. Conductivity, Seebeck coefficient and power factor vs. thickness from single layer devices using sequential doping. Lines are guides to the eye. b. Same for bulk doping vs. F4TCNQ doping ratio from $10^{-5}$ to $10^{-2}$ molar ratio at thickness $\sim 150 \mathrm{~nm}$. Data points are averages over multiple devices, the error bar on the conductivity is typically smaller than the symbol size. Adapted with permission from Paper VI.

The undistorted morphology of the P3HT films after F4TCNQ deposition is demonstrated by the surface topography images obtained by atomic force microscopy (AFM) images shown in Figure 3.8. The pure P3HT film shows a smooth morphology with a roughness $R_{q}=0.92 \mathrm{~nm}$; after spin-coating a single layer of $\mathrm{F}_{4} \mathrm{TCNQ}$ this becomes $R_{q}=0.82 \mathrm{~nm}$.

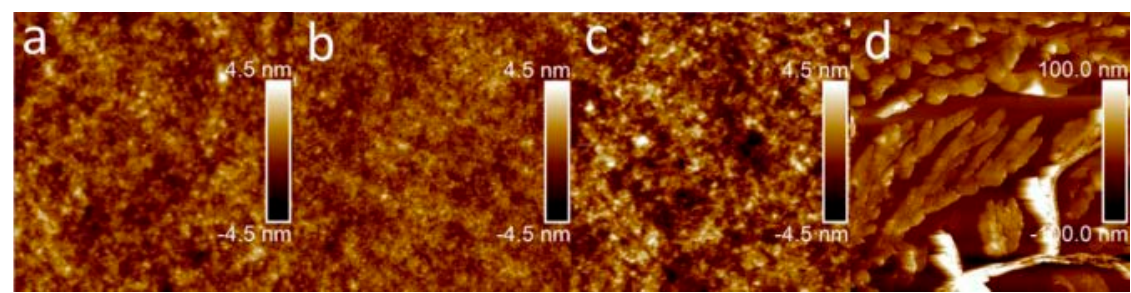

Figure 3.8 AFM height images for a. pure P3HT, d. pure $\mathrm{F}_{4} \mathrm{TCNQ}$ and surface doped P3HT with b. (1) and c. (3) spin-coated $\mathrm{F}_{4}$ TCNQ layers. The scan size is $2 \times 2 \mu \mathrm{m}$ in a-c and $20 \times$ $20 \mu \mathrm{m}$ in $\mathrm{d}$;

Having a high power factor PF is necessary for efficient TEG. In comparison to conventional bulk doping, sequential doping can achieve higher conductivity by preserving the morphology, such that the power factor can improve over 100 times. However, to achieve TEG with high output power, not only a high $\mathrm{PF}$ is needed, but also having a significant active layer thickness is very important. The output power $(\mathrm{P})$ can be calculated as

$$
P=\mathrm{PF} \Delta T^{2} A / l
$$

where the area $A=w t$ with $w$ the width of the contacts, $t$ the film thickness and $l$ the channel length, $\Delta T$ is the temperature difference over the active layer. ${ }^{36}$ 

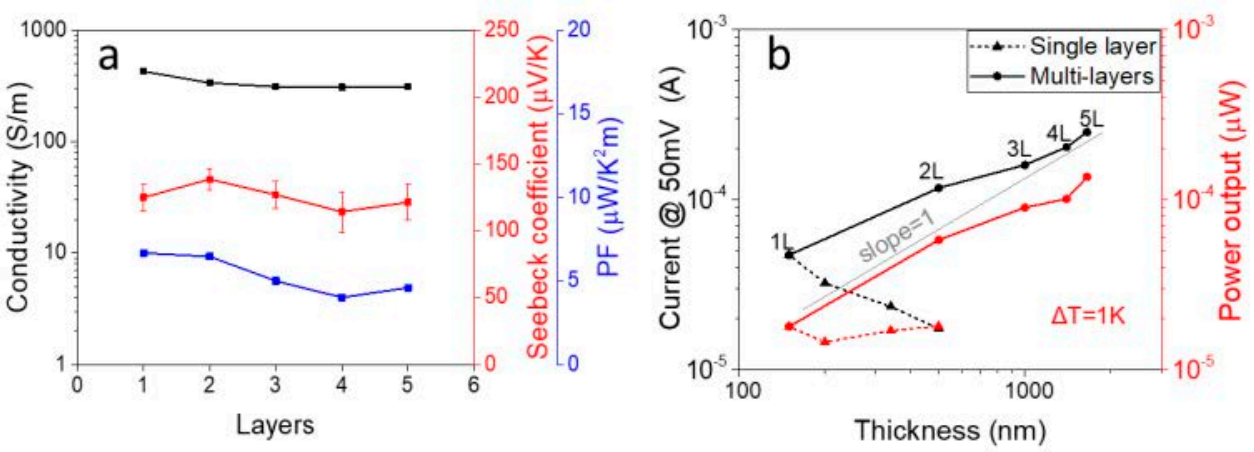

Figure 3.9 a. Conductivity, Seebeck coefficient and power factor vs. number of deposited P3HT:F4TCNQ layers; b. current at $50 \mathrm{mV}$ and estimated power output at $\Delta T=1 \mathrm{~K}$ (solid line + circles: multilayer; dashed line + triangles: single- layer), vs. layer thickness. Data points are averages over multiple devices, the error bar on the conductivity is smaller than the symbol size. Adapted with permission from Paper VI.

In Figure 3.7 (a) we show that for thicker films the electrical conductivity decreases significantly while the Seebeck coefficient slightly increases. The output power is almost constant while significantly sacrificing PF as seen in Figure 3.9 (b). We developed a simple way to fabricate multi-layer devices by sequential doping without significantly sacrificing electrical conductivity and PF as illustrated in Figure 3.7 above; the results are shown in Figure 3.9 ( $a$ and $b$ ). Both the current and the power output almost linearly increase by an order of magnitude in going from 1 to 5 layers with very minor changes in surface morphology, shown in Figure 3.8 (c) for the triple-doped film $\left(R_{q}=1.15 \mathrm{~nm}\right)$.

\subsubsection{Vapor doping}

Vapor doping is similar to sequential doping in the sense that dopant molecules are deposited (by thermal evaporation) onto a previously deposited semiconductor film. Patel et al. showed that PBTTT vapor-doped with $\mathrm{F}_{4}$ TCNQ on OTS-treated substrates can achieve a high electrical conductivity of $6.7 \times 10^{4} \mathrm{~S} / \mathrm{m}$ and a power factor around $120 \mu \mathrm{W} / \mathrm{m} \cdot \mathrm{K}^{-2}$. Unfortunately, the active layer thickness was just $\sim 25 \mathrm{~nm}$ which limits the absolute power output and it is unsure whether the method can be used for significantly thicker layers, c.f. Figure 3.7(a). Moreover, the methods seem neither suitable for 'fast' large-area products manufacture. ${ }^{36}$

\subsubsection{Sink doping}

In comparation with 'fast' spin-coated sequential doping, sink doping can achieve optimal device performance by submerging the pre-fabricated semiconductor film in a solution containing the dopant. With time the dopant molecules permeate into the film to reach the 
maximum doping efficiency. Hwang et al. put pre-made $\mu$ m-thick P3HT films in $\mathrm{Fe}^{3+}$ $\operatorname{tos}_{3} \cdot 6 \mathrm{H}_{2} \mathrm{O}$ containing solution and acquired a high conductivity around $5.5 \times 10^{3} \mathrm{~S} / \mathrm{m}$ and a high power factor around $30 \mu \mathrm{W} / \mathrm{m} \cdot \mathrm{K}^{-2}$. Unfortunately, the method requires extended time $(\sim 1 \mathrm{hr})$ to allow the dopants to diffuse throughout the entire film. ${ }^{99}$

\subsubsection{Overview}

Above we introduced several doping methods and found that the doping effect on thermoelectric properties significantly varies with doping procedure. Some of the thermoelectric properties obtained from the different discussed methods are summarized in Table 3.1.

Table 3.1 Summary of thermoelectric properties obtained for the F4TCNQ dopant with different semiconductors by solution processing

\begin{tabular}{|c|c|c|c|c|c|c|}
\hline Material & Dopant & $\begin{array}{c}\text { Electrical } \\
\text { conductivity } \\
(\mathrm{S} / \mathrm{m})\end{array}$ & $\begin{array}{c}\text { Seebeck } \\
\text { coefficient } \\
(\mu \mathrm{V} / \mathrm{K})\end{array}$ & $\begin{array}{c}\begin{array}{c}\text { Power } \\
\text { factor } \\
\left(\mu \mathrm{W} / \mathbf{m K}^{2}\right)\end{array}\end{array}$ & Processing & Ref. \\
\hline РBTTT & $\mathrm{F}_{4} \mathrm{TCNQ}$ & $\begin{array}{c}67000 \pm \\
400\end{array}$ & $42 \pm 30$ & $120 \pm 30$ & $\begin{array}{l}\text { Vapor doping on } \\
\text { an OTS-treated } \\
\text { substrate }\end{array}$ & Ref. $^{36}$ \\
\hline P3HT & $\mathrm{F}_{4} \mathrm{TCNQ}$ & $\sim 662$ & $\sim 114$ & $\sim 8.7$ & Sequential doping & Ref. $^{31}$ \\
\hline P3HT & $\mathrm{F}_{4} \mathrm{TCNQ}$ & $\sim 2200$ & $\sim 60$ & $\sim 8$ & $\begin{array}{l}\text { High temperature } \\
\text { rubbing } \mathrm{P} 3 \mathrm{HT}\end{array}$ & Ref. $^{121}$ \\
\hline P3HT & $\mathrm{F}_{4} \mathrm{TCNQ}$ & $\sim 1000$ & $\sim 90$ & $\sim 8.1$ & $\begin{array}{l}\text { P3HT film sink in } \\
\mathrm{F}_{4} \mathrm{TCNQ} \text { solution }\end{array}$ & Ref. ${ }^{99}$ \\
\hline P3HT & $\mathrm{F}_{4} \mathrm{TCNQ}$ & $\sim 30$ & $\sim 60$ & $\sim 0.1$ & $\begin{array}{l}\text { Bulk doping by } \\
\text { adding PEO to } \\
\text { form ternary } \\
\text { system }\end{array}$ & Ref. ${ }^{119}$ \\
\hline P3HT & $\mathrm{F}_{4} \mathrm{TCNQ}$ & $\sim 0.04$ & $\sim 400$ & $\sim 0.006$ & Bulk doping & Ref. $^{104}$ \\
\hline
\end{tabular}




\subsection{Morphology influence}

Morphology heavily influences the thermoelectric properties in a doped semiconductor system. Patel et al. reported that vapor-doped films having over two orders of magnitude higher electrical conductivity than that of solution-doped films because of a better, i.e. less negatively affected, morphology. In particular, they indicated a large orientational correlation length resulting in high apparent charge carrier mobility in vapor-doped films. ${ }^{36}$

We note that the morphology influences the results not only via different processing methods, but also via the thickness dependence of the anisotropic transport, especially in crystal-like semiconductors like rr-P3HT. Recently, Huang et al. found a strong thickness dependence of the anisotropy of rr-P3HT; specifically, between $\sim 5$ and $\sim 100 \mathrm{~nm}$ the fraction of (substrateinduced) edge-on crystals was found to decay strongly. ${ }^{99}$ This is consistent with our observations on conductivities for film thicknesses in the range of 25-150 nm for sequential doped P3HT films as shown in Figure 3.7 (a). We attribute the 'oscillating' conductivity to a trade-off of the above-mentioned thickness dependence of the anisotropic transport (in-plane vs out-plane conductivity) and the molecular doping effect. The first drop in in-plane conductivity is attributed to a reduced $\pi-\pi$ stacking in the current direction. The upturn and subsequent second decay of the conductivity is attributed to the (decaying) mean doping concentration passing through an optimal value. ${ }^{31}$ Since molecular doping has both a beneficial effect on the mobility, through state filling, as well as a detrimental effect, through Coulomb scattering by ionized dopants and possibly some morphology degradation, one may anticipate an optimal thickness at which the concentration (profile) is optimal. 


\subsection{Dopants}

Here we give a brief overview of some commonly used dopants with chemical structures. In our work, we basically use $\mathrm{F}_{4} \mathrm{TCNQ}$ and N-DPBI as p-type and n-type dopant, respectively.

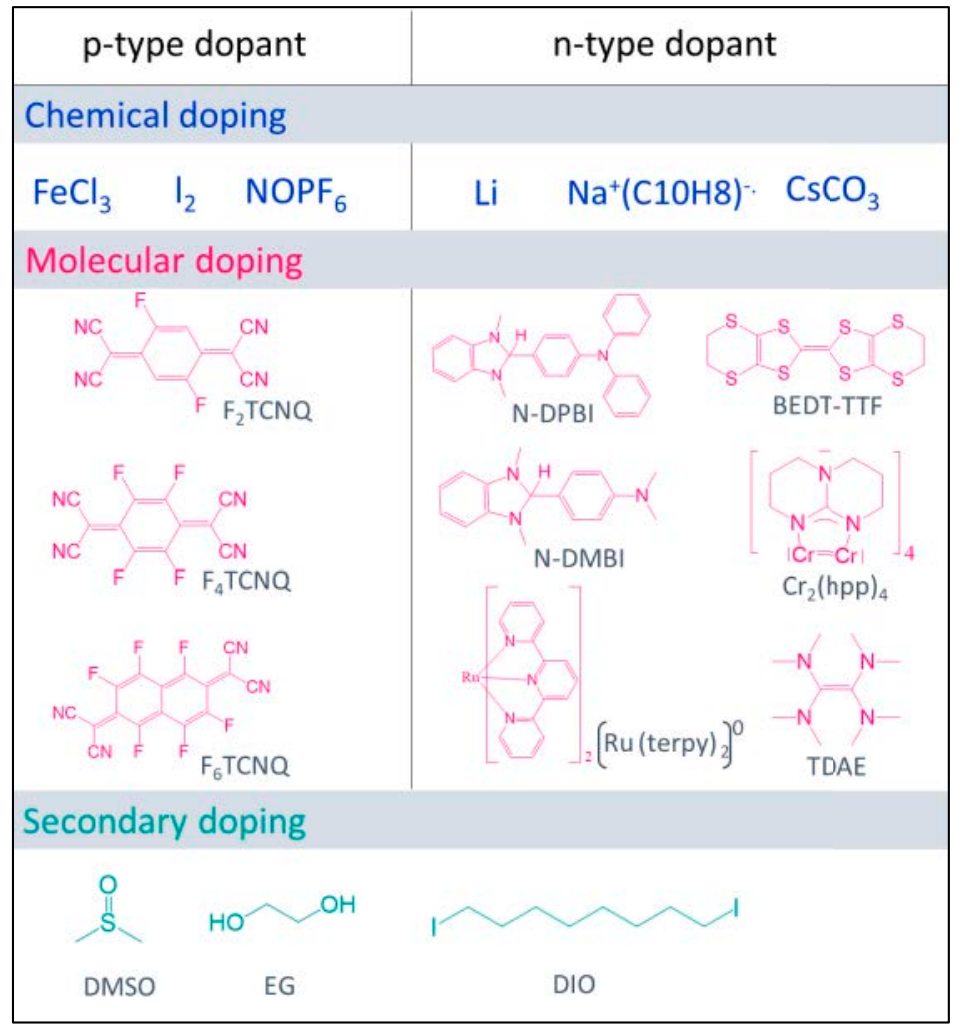

Figure 3.10 Chemical structures of some commonly used dopants for different doping types. 


\subsection{Impact of the dopant counterion}

\subsubsection{Impact on density of states}

Molecular doping has both a beneficial effect on the mobility, through state filling, as well as a detrimental effect, through Coulomb scattering by ionized dopants and possibly some morphology degradation. ${ }^{53,116,122,123}$ Consistent with this trade-off of the two effects, it has in $\mathrm{F}_{4} \mathrm{TCNQ}$ doped P3HT devices been experimentally observed that the hole mobility first decreases with increasing doping level at low to moderate doping concentrations, before increasing steeply at higher doping levels. ${ }^{116}$ We attribute the sublinear evolution of mobility to dopant counterions perturbing the intrinsic DOS distribution of organic semiconductors, as discussed below and in Paper $\mathbf{I}$.

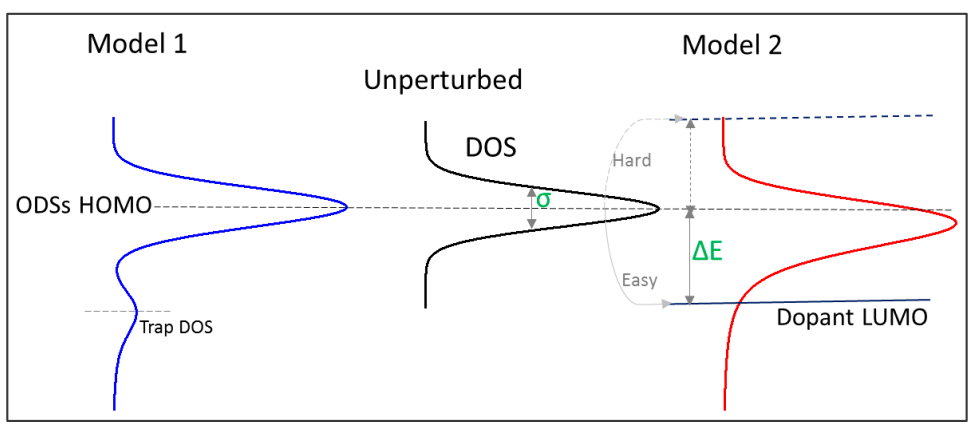

Figure 3.11 Illustration of the shape of DOS distribution used in Model I (Eq. 3.2, blue), undoped (Eq. 3.1, black), and in Model II (Eq. 3.4 with $\Delta E=0$, red).

In previous works, different approximations have been made for the impact dopant ions have on the host DOS distribution. Typically, models describe this influence by assuming counterions act as Coulombic traps. In this thesis, we have considered two models. Both analytical models are developed by Arkhipov ${ }^{124,125}$ and are based on the idea that the original Gaussian DOS

$$
g_{i}(E)=\frac{N}{\sqrt{2 \pi} \sigma_{D O S}} \exp \left(-\frac{\left(E-E_{i}\right)^{2}}{2 \sigma_{D O S}^{2}}\right)
$$

is modified by low-lying dopant-induced states. In Model I a discrete, second Gaussian-shaped DOS of traps sits below the intrinsic DOS of the host materials as shown in Figure 3.11. In Model II introduced ions cause a continuous, essentially exponential tail below the intrinsic DOS.

Model I follows the work of Arkhipov in Ref. ${ }^{124}$ with DOS 


$$
g(E)=\frac{N-N_{d}}{N_{i}} g_{i}(E)+\frac{N_{d}}{N} g_{i}\left(E+\frac{q^{2}}{4 \pi \varepsilon_{0} a}+U_{m}\right)
$$

with $N_{d}$ the doping density and $a$ the typical distance between the dopant ion and the trapped charge carrier. $U_{m}$ is the maximum of the net potential formed by the overlapping Coulomb potentials of neighboring dopant ions in the presence of an externally applied electrostatic field. Model II employs a DOS that was obtained by Arkhipov et al. ${ }^{125}$ as:

$$
g(E)=A \int_{-\infty}^{0} \frac{d E_{C}}{E_{C}^{4}} \exp \left(\frac{A}{3 E_{C}^{3}}\right) g_{i}\left(E-E_{C}\right)
$$

with $A=\frac{4 \pi q^{6} N_{d}}{\left(4 \pi \varepsilon_{0} \varepsilon_{r}\right)^{3}}, E_{C}(r)=-q^{2} / 4 \pi \varepsilon_{0} \varepsilon_{r} r$, and $\varepsilon_{r}$ the relative dielectric constant of the semiconductor, here taken as 3.6. In its original shape, this expression cannot account for energy level offsets between the dopant and the semiconductor, $\Delta E=E_{d}-E_{i}$ with $E_{d}$ the relevant energy level of the dopant. Defining $E_{1}=E_{C}\left(N_{i}^{-1 / 3}\right)$ as the Coulomb energy one lattice constant away from the ionized dopant we write for the DOS

$$
\begin{gathered}
g(E)=\left(1-\frac{4 \pi N_{d}}{3 N_{i}}\right) \frac{g_{1}(E)}{\int_{-\infty}^{0} d E g_{1}(E)}+\frac{4 \pi N_{d}}{3 N_{i}} \frac{g_{2}(E)}{\int_{-\infty}^{0} d E g_{2}(E)} \\
g_{1}(E)=A \int_{E_{1}}^{0} \frac{d E_{C}}{E_{C}^{4}} \exp \left(\frac{A}{3 E_{C}^{3}}\right) g_{i}\left(E-E_{C}\right) \\
g_{2}(E)=A \int_{-\infty}^{E_{1}} \frac{d E_{C}}{E_{C}^{4}} \exp \left(\frac{A}{3 E_{C}^{3}}\right) g_{i}\left(E-\Delta E-E_{C}\right)
\end{gathered}
$$

To examine which model best describes the impact of dopant counterions on the DOS of host materials, we calculated the evolution of the DOS from the two models with dopant concentrations from $10^{-5}$ to $10^{-1}$. Comparing with the results of Monto Carlo simulations, we found that only Model II accurately represents the DOS distributions with varying dopant fractions as seen in Figure 3.12.
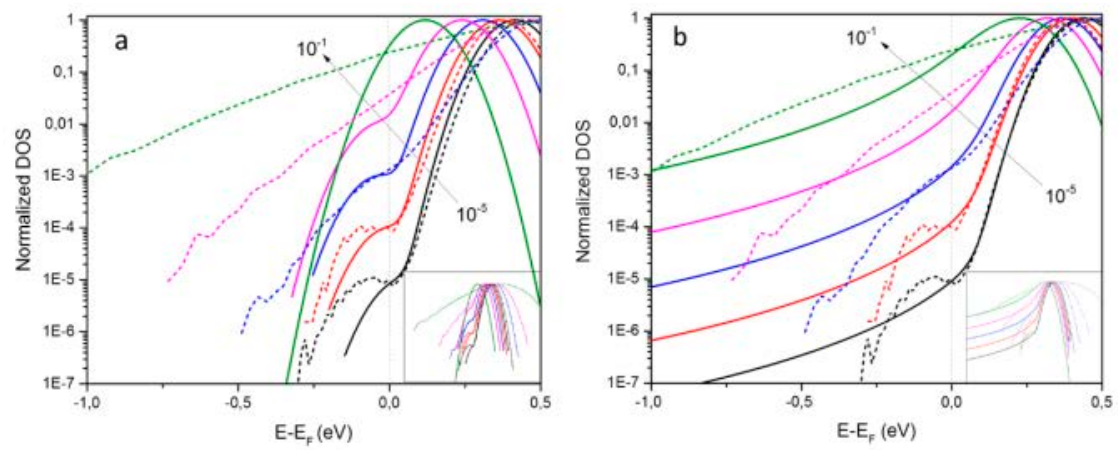
Figure 3.12 DOS for different doping concentrations from a. model I (Eq. 3.2, solid lines); b. model II (Eq. 3.4, solid lines), and MC (dashed lines) with the standard parameters: $v_{0}=1 \mathrm{e}-11$ $\mathrm{s}^{-1} ; a_{N N}=1.8 \mathrm{~nm} ; \sigma_{D O S}=0.075 \mathrm{eV} ; T=300 \mathrm{~K}$; and energy level difference $\Delta E=0$. The insets show the full energy scale. All DOS areas are normalized to unity. Adapted with permission from Paper I.

\subsubsection{Impact on mobility/conductivity}

On the basis of Model II, we calculated the mobility varying various parameters like energetic disorder, energy level different, temperature and so on, from ultralow dopant concentration 10${ }^{5}$ up to $10^{-1}$. The mobilities calculated in Model II have a good agreement with the results of kMC simulations in a wide range of parameters and doping concentration, as seen in Figure 3.13. We attribute the agreements to the similar DOS distributions, c.f. Figure 3.12. More details on specific parameter impact are discussed in Paper $\mathbf{I}$.
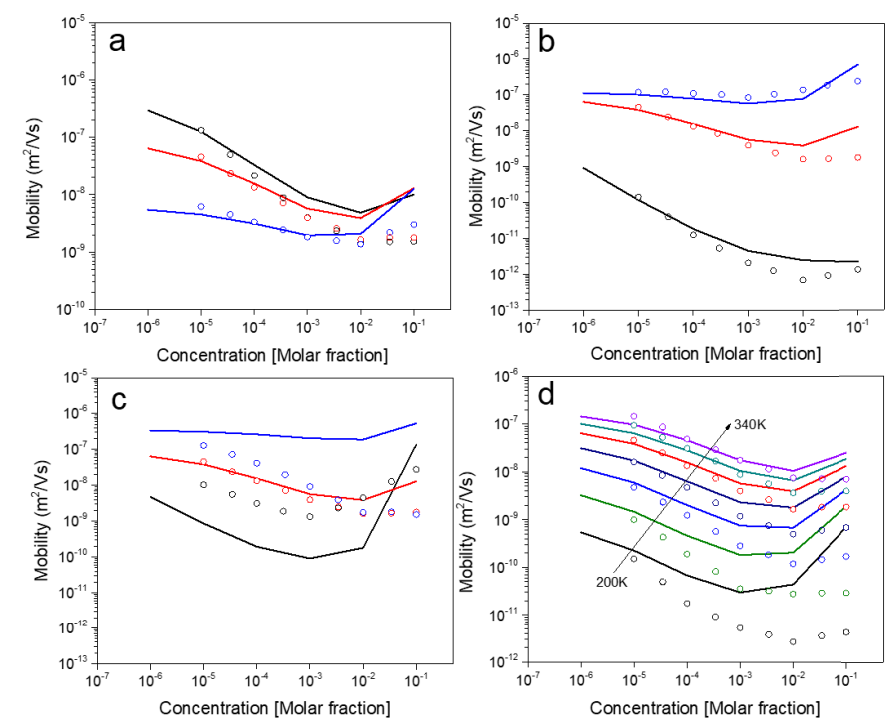

Figure 3.13 Dependence of hole mobility on doping concentration from model II (solid lines), and MC (dots) for different parameters: a. energetic disorder: $\sigma_{D O S}=0.05 \mathrm{eV}$ (black), $0.075 \mathrm{eV}$ (red) and $0.1 \mathrm{eV}$ (blue); b. energy level difference: $\Delta E=-0.2 \mathrm{eV}$ (black), $0 \mathrm{eV}$ (red) and $0.3 \mathrm{eV}$ (blue); c. inter-site distance $a_{N N}=1.0 \mathrm{~nm}$ (black), $1.8 \mathrm{~nm}$ (red), $3.0 \mathrm{~nm}$ (blue); d. temperature: $T=200 \mathrm{~K}, 225 \mathrm{~K}, 250 \mathrm{~K}, 275 \mathrm{~K}, 300 \mathrm{~K}, 320 \mathrm{~K}, 340 \mathrm{~K}$. Adapted with permission from Paper I. 

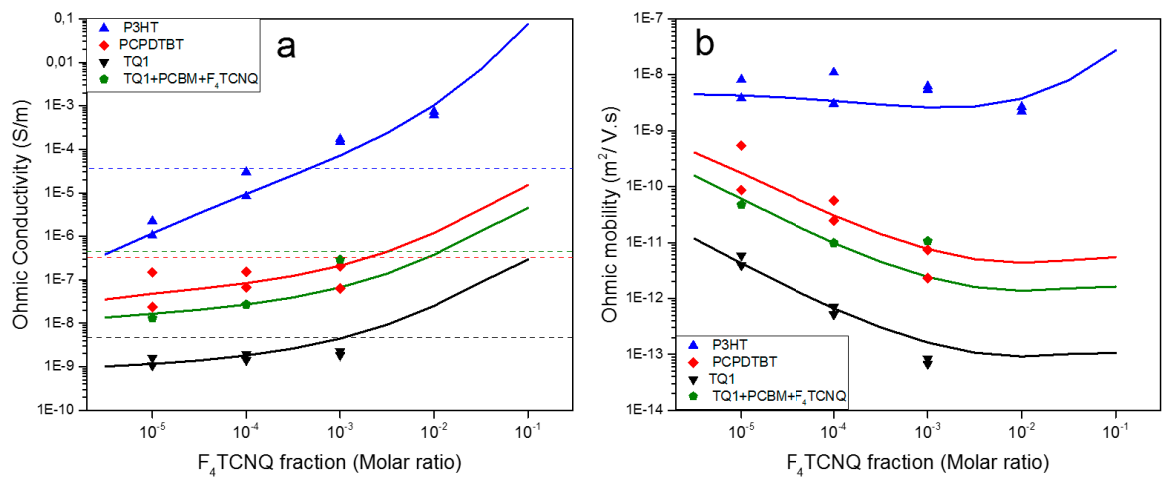

Figure 3.14 Dependence of a. the Ohmic conductivity and b. the hole mobility derived from that for different polymers with various $\mathrm{F}_{4}$ TCNQ doping concentration. Symbols represent experimental data of individual devices of TQ1 (black down-triangle, thicknesses $255 \mathrm{~nm}$ and $327 \mathrm{~nm}$ ), PCPDTBT (red rhombus, thicknesses $230 \mathrm{~nm}$ and $260 \mathrm{~nm}$ ), P3HT (blue up-triangle, thicknesses $265 \mathrm{~nm}$ and $360 \mathrm{~nm}$ ), and TQ1:PCBM (pentagon, 1:1wt), dashed lines in (a) show the conductivity of pure CPs with the same color; solid lines represent analytical data from the model with the following parameters: P3HT (blue): $\Delta E=0.24 \mathrm{eV}$ PCPDTBT (red) $\Delta E=-0.1$ $\mathrm{eV}$; TQ1 (black) $\Delta E=-0.23 \mathrm{eV}$; TQ1+PCBM (olive): $\Delta E=-0.16 \mathrm{eV}$. For all materials $v_{0}=$ $5 \mathrm{e} 9 \mathrm{~s}^{-1}$ and $\sigma_{D O S}=0.075 \mathrm{eV}$ were used. Adapted with permission from Paper II.

To experimentally inverstigate the model validity, we measured conductivity and mobility of different conjugated polymers (P3HT, PCPDTBT and TQ1), bulk doped by $\mathrm{F}_{4} \mathrm{TCNQ}$, covering a wide range of concentration. Figure 3.14 shows a very good agreement between experiments and Model II's results with doping concentration from $10^{-5}$ to $10^{-3}$, that a decrease in mobility, also found the 'similar trend' by other researchers. ${ }^{116,124,126}$

\subsubsection{Impact on Seebeck coefficient and power law}

In section 1.3 we mentioned that the magnitude of the Seebeck coefficient is determined by the energy gap between the transport energy $E_{t r}$ and the Fermi energy $E_{F}$. The choice of transport model has no influence on the location of $E_{F}$, but does so on $E_{t r}$. 


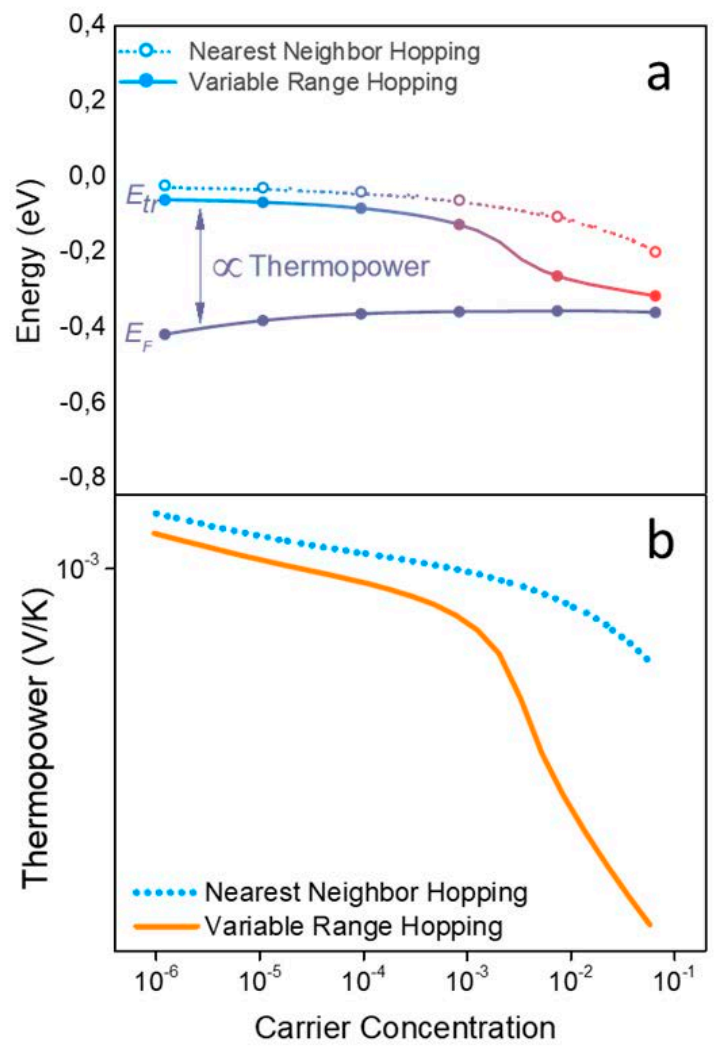

Figure 3.15 a. Fermi energy $E_{F}$ and transport energy $E_{t r}$ calculated for VRH and NNH leading to the carrier concentration dependence of thermopower $\mathbf{b}$. Adapted with permission from Paper IV.

Following our Model II, we calculated the doping dependence of the transport energy and the Fermi energy with different charge hopping models, i.e. nearest neighbor hopping vs. variable range hopping. As shown in Figure 3.15 (a), $E_{F}$ does indeed not depend on the choice of hopping model, whereas $E_{t r}$ does, details are explained in Paper IV. The difference in thermopower in Figure 3.15 (b) directly reflects the difference between NNH and VRH models at doping concentrations above $10^{-3}$ due to the latter having more freedom to adopt (via the variable hopping range) to changes in DOS shape. 


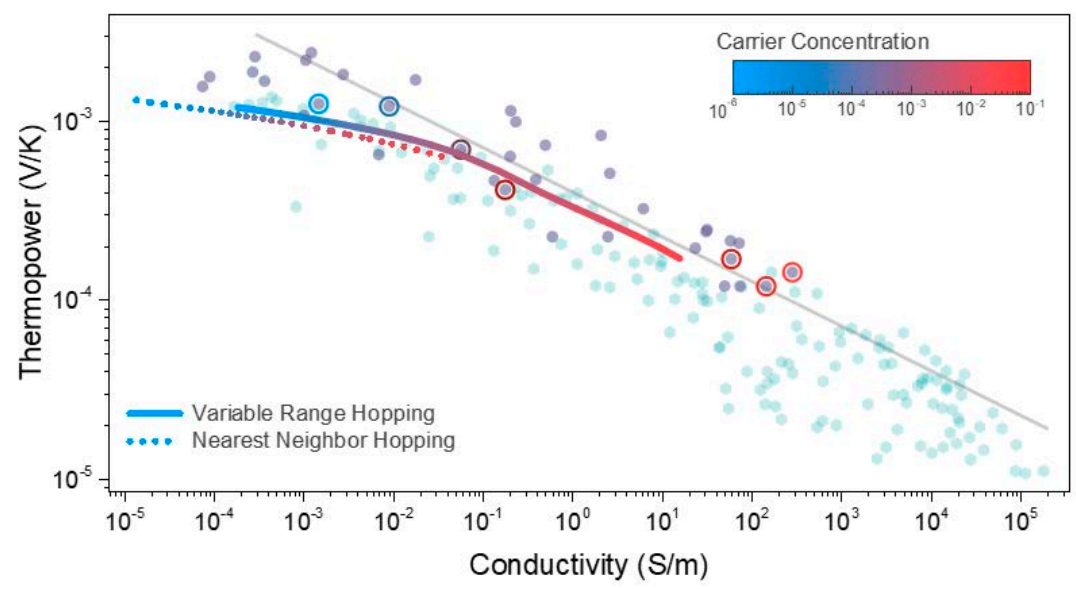

Figure 3.16 Thermopower vs. conductivity of doped disordered organic semiconductors. ptype experimental data from our work (grey dots) and Ref. ${ }^{44}$ (green dots). Simulations from $\mathrm{NNH}$ (dashed line) and VRH (bold solid line). The grey line is the empirical -1/4 power law. Adapted with permission from Paper IV.

We combined the calculated conductivity and thermopower from both NNH and VRH models with experimental data from our own group and literature. ${ }^{127}$ Figure 3.16 shows that the VRH model gives a good agreement with the experimental data and follows an experimentally observed 'universal' $-1 / 4$ power law relationship. ${ }^{44}$ We note that the VRH model accurately reproduces the 'universal' power law behavior due to the fact that the Seebeck coefficient and the conductivity are both dominated by the 'universal' dopant counterion-induced tail of DOS at high doping concentration. ${ }^{127}$

Interestingly, it was stated in Ref. ${ }^{44}$ that VRH models could not explain the universal power law. The reason for the stated failure is the fact that in the used expression the DOS was assumed to be constant and independent of doping concentration. Alternatively, this behavior has been fitted using an empirical conductivity distribution function by Kang and Snyder. ${ }^{28}$ 


\section{DOS engineering for Seebeck coefficient}

\subsection{DOS engineering in $\mathbf{p}-\&$ n-type materials}

In Chapter 3 we have discussed how the dopant broadens the intrinsic DOS of the host material by introducing a deep tail due to ionized dopants acting as Coulombic trap. Here we address the question how the thermoelectric properties are affected when two CPs are blended and a 'double Gaussian' DOS is created.
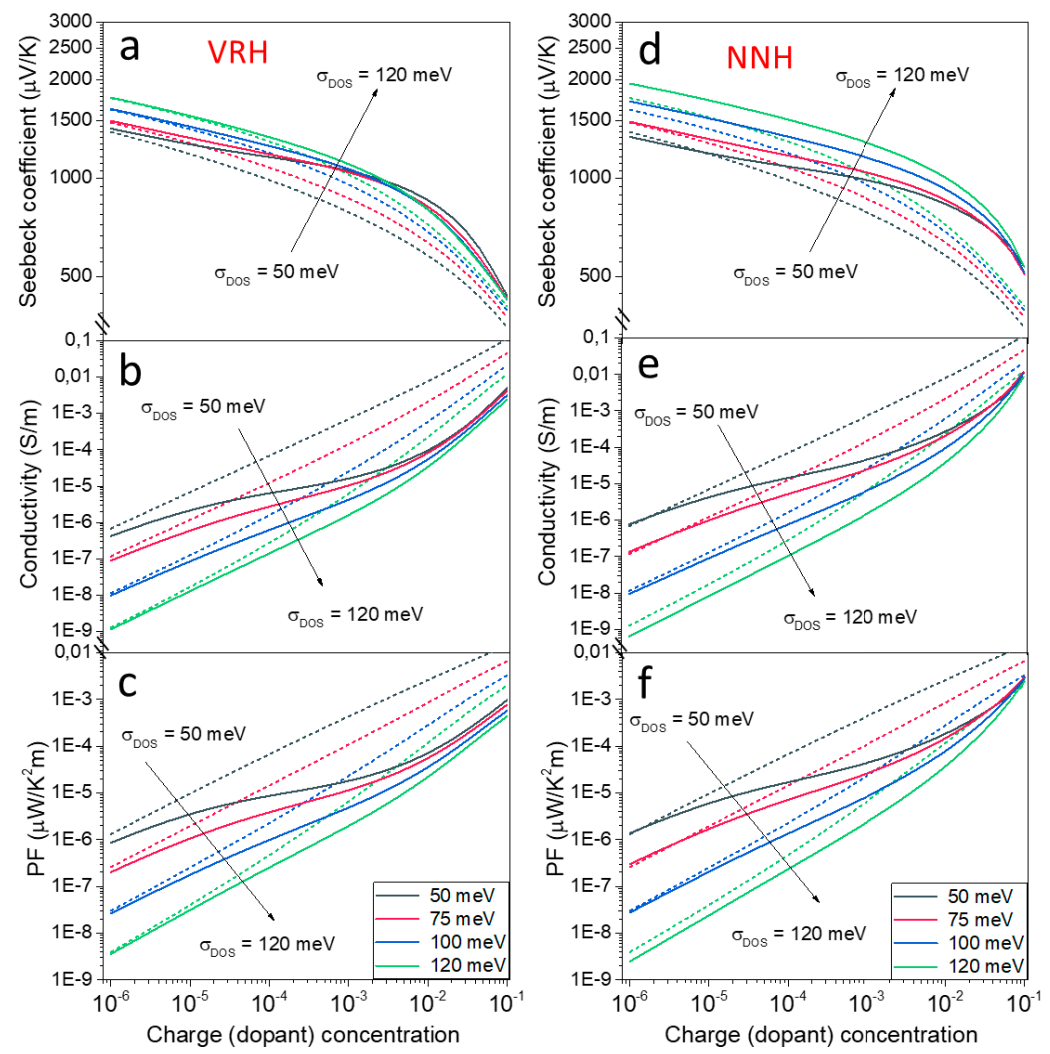

Figure 4.1 Seebeck coefficient, conductivity, and power factor for both pristine and doped OSC calculated from Model II with variable range hopping (a-c) and nearest neighbor hopping (de). Parameters used $v_{0}=10^{-13} \mathrm{~s}^{-1} ; a_{N N}=1.8 \mathrm{~nm} ; \sigma_{D O S}=50-120 \mathrm{meV} ; T=300 \mathrm{~K}$; and $\alpha=2 \mathrm{e} 9$ $\mathrm{nm}^{-1}$.

Researchers have been investigating both theoretically and experimentally the Seebeck coefficient and electrical conductivity. ${ }^{8,35,104,128-134}$ Particularly, in the theoretical works it is commonly assumed that the DOS is of a given shape and consists of a single Gaussian peak only. While those results indicate the importance of the DOS width and state filling, they hardly address the freedom one has to tune thermoelectric properties. Basically, within a VRH model 
framework with a fixed, single Gaussian DOS, only disorder and concentration are considerable parameters to tune the Seebeck coefficient and electrical conductivity. The other parameters in the system either have a limited effect, notably the dielectric constant and the typical inter-site distance, or act as a trivial scaling factor, notably the attempt-to-hop frequency.

On basis of the model we introduced before (section 3.6), we first indicate how the thermoelectric properties are expected to vary with disorder and concentration in a single Gaussian-shaped DOS. Figure 4.1 shows Seebeck coefficient, electrical conductivity and PF calculated by the VRH and NNH versions of the model for both the pristine and the doped cases, respectively. Indeed, the PF dramatically increases with increasing charge concentration resulting from the significant improvement in conductivity that dominates over the loss in thermopower. The energetic disorder has a considerable impact on the Seebeck coefficient for the pristine case, i.e. an unperturbed DOS, increasing the disorder leads to an increase in $S$ at any given concentration due to the increasing gap between the Fermi and transport energies, as explained in Chapter 2. However, large disorder still causes a loss in PF due to the strong tradeoff between $S$ and conductivity. Considering the ultralow intrinsic electrical conductivity there is quite limited freedom to tune the thermoelectric properties for pristine OSCs: the simple design rule is that low disorder and high charge concentrations give the best power factors.

As explained in the previous chapter, charge transport is heavily affected by the induced tail of DOS due to dopant ions at high doping concentrations. In particular, the DOS broadening results in a negligible effect of the intrinsic DOS width, i.e. the thermoelectric properties become independent of pristine disorder. ${ }^{127}$ For the parameters used in these simulations, which we think are representative of most conjugated polymers, this happens for concentrations higher than $\sim 10^{-2}$. The consequence is that the 'realistic' design rule is that high charge/dopant concentrations are best. Evidently, this should be achieved without destroying the pristine morphology which in our model would negatively affect the conductivity prefactor via the attempt-to-hop frequency.

While there are quantitative differences between the VRH and NNH model calculations in Figure 4.1, the above holds equally for both. As a consequence, one has very limited possibilities to tune and improve thermopower, conductivity and power factor by varying the disorder as increasing the doping concentration will 'overrule' this at the relevant high dopant concentrations, as seen in Figure 4.1.

We note that the differences in $S$ and conductivity between the pristine (dashed lines) and doped (solid lines) OSCs at increasing charge or dopant concentration are, equivalently, attributable to the fraction of free charges. While all charges are free charges in the pristine case, the fraction of free charges decreases with increasing dopant concentration for dopant-induced charges as we discussed in Chapter 3.2. The difference between the two models is further explored in Paper IV. As above, for a given single DOS, there is a limitation to tune the thermoelectric properties by varying disorder and concentration in both pristine and doped OSCs. 


\subsubsection{DOS shape with two materials}

Mahan and Sofo theoretically proposed the concept of a delta-shaped transport distribution, situated somewhere above the Fermi energy, to maximize the thermoelectric properties. ${ }^{135}$ Later Katz et al. experimentally confirmed this idea of DOS engineering in $\mathrm{F}_{4} \mathrm{TCNQ}$ doped P3HT by blending in a small fraction of P3HTT, increasing S from $\sim 580$ to $700 \mu \mathrm{V} / \mathrm{K}$ while preserving conductivity. ${ }^{104}$ We revisited this strategy of DOS engineering and deepened the physical understanding of this concept for systems consisting of two blended OSCs. More details than discussed below can be found in Paper III, V and VII.

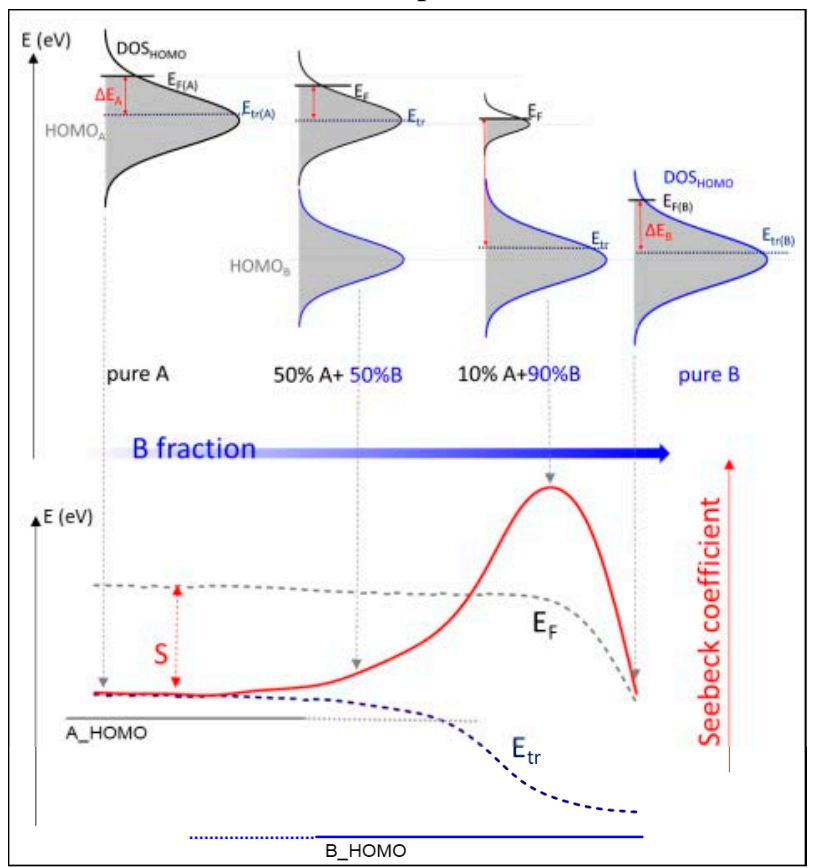

Figure 4.2 Illustration of DOS engineering to achieve a high Seebeck coefficient. Upper part: hole density of states for a binary mixture A:B. White and shaded regions indicate hole- and electron-filled parts of the DOS, respectively. Lower part: corresponding relative positions of the Fermi energy $E_{F}$ and the transport energy $E_{t r}$ and the Seebeck coefficient $S$ that is proportional to their difference. Adapted with permission from Paper III.

On the assumption that no interaction occurs between the intrinsic DOS of CPs, the total DOS distribution of two blended CPs just combines the separated DOS distributions at the energy of the respective HOMO (or LUMO) levels; the height of the DOS sub-peaks is directly proportional to the respective fraction. Figure 4.2 (for p-type) shows the evolution of total HOMO DOS for different materials $\mathrm{A}$ and $\mathrm{B}$, varying the blend fraction. As explained in Chapter 2, $E_{F}$ is determined by the charge concentration and $E_{t r}$ is related to the part of the total DOS that supports or carries the charge transport. More details are explained in Paper III. With tuning the A:B ratio, somewhere the $E_{F}$ and $E_{t r}$ will be located on different materials, 
leading to a much enhanced splitting between the two which allows to achieve a high Seebeck coefficient as illustrated in Figure 4.2.

\subsubsection{Impact on thermoelectric properties}

To test the concept in Figure 4.2, we both experimentally and theoretically investigated the conductivity, Seebeck coefficient and power factor for blends based on $\mathrm{P}_{3} \mathrm{HT}_{1-x}: \mathrm{PTB}_{x}$ with $x$ the fraction covering the range between 0 and $100 \%$, as seen in Figure 4.3.
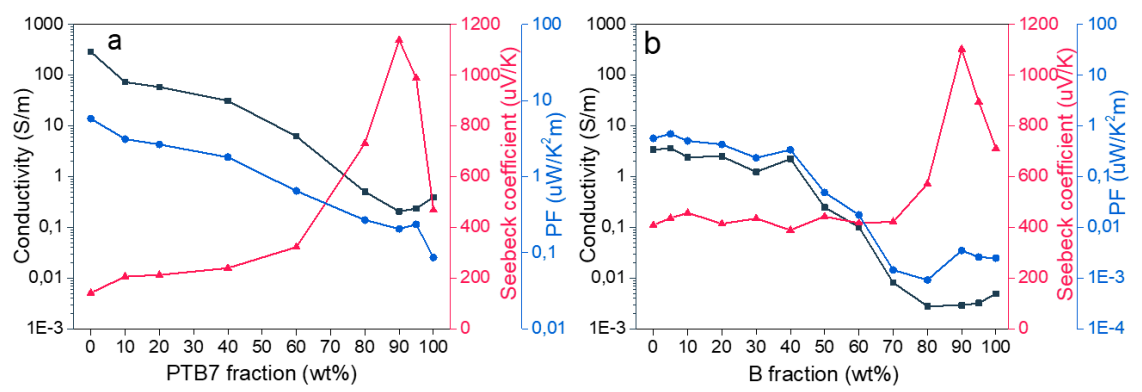

Figure 4.3 Conductivity, Seebeck coefficient and power factor for a. experimental data for $\mathrm{P}_{3} \mathrm{HT}_{x}$ :PTB7 $1-x$; b. NNH kinetic Monte Carlo simulations for $\mathrm{A}_{x}: \mathrm{B}_{1-x}$ mixtures with HOMO energy differences of $0.3 \mathrm{eV}$, dependent on active layer composition. Parameters used $v_{0}=10^{-}$ ${ }^{13} \mathrm{~s}^{-1} ; a_{N N}=1.8 \mathrm{~nm} ; \sigma_{D O S}=75 \mathrm{meV} ; T=300 \mathrm{~K} ; \mathrm{HOMO}_{\mathrm{A}}-\mathrm{LUMO}_{\text {dopant }}=0.24 \mathrm{eV}$. Adapted with permission from Paper III.

The kinetic MC simulations were run with parameters related to the taken experimental system, employing a nearest-neighbor hopping model described in detail in Paper III. The results of the simulations (panel b) show a good agreement with the experiments (panel a). We found that an obvious peak in Seebeck coefficient of $\sim 1200 \mu \mathrm{V} / \mathrm{K}$ shows up at $\mathrm{P}_{3 \mathrm{HT}_{10}}: \mathrm{PTB}_{90}$ and $\mathrm{A}_{10}: \mathrm{B}_{90}$, respectively. Surprisingly, the measured conductivity is much higher than the calculated one at the same doping concentration, which is possibly attributed to the sequential doping effect described in Chapter 3.3.

For the (simulated) single DOS case discussed in Figure 4.1, the Seebeck coefficient is $\sim 500$ $\mu \mathrm{V} / \mathrm{K}$ at a conductivity $\sim 10^{-3} \mathrm{~S} / \mathrm{m}$ for both pristine and doped OSCs, c.f. panels a and $\mathbf{d}$ in Figure 4.1. For the simulated blended system, the Seebeck coefficient is $1200 \mu \mathrm{V} / \mathrm{K}$ at the same conductivity. This is consistent with the assertion by Mahan and Sofo and the finding by Katz et al. that blending of CP makes it possible to achieve higher $S$ at comparable conductivities as compared to a single-component system. We should stress that the simulations as done in Figure 4.1 for single OSCs show an accurate agreement with experiments in section 3.3.2, see also Paper VII. Further details and more p- and n-type material combinations are shown in Papers III and V. 


\subsection{Morphological effect on thermoelectrics}

Morphology heavily influences the performance of organic electronic devices in host-guest systems. ${ }^{136-138}$ The distribution of localized sites in two materials blended together is more complicated, and has larger consequences for performance than that in a single material. When the two materials have quite different HOMO or LUMO energy levels, different blend morphologies will cause different behavior - imagine a low-LUMO material in a high-LUMO matrix; when the former forms isolated clusters these will act as charge carrier traps, but when it forms a continuous phase, charges will be transported entirely in the guest and not 'see' the matrix. In Paper VII we investigated in more detail how morphology in blended OSC impacts the thermoelectric properties. The mechanism shown in Figure 4.4 briefly explains the influence of morphology on Seebeck coefficient in such blends.

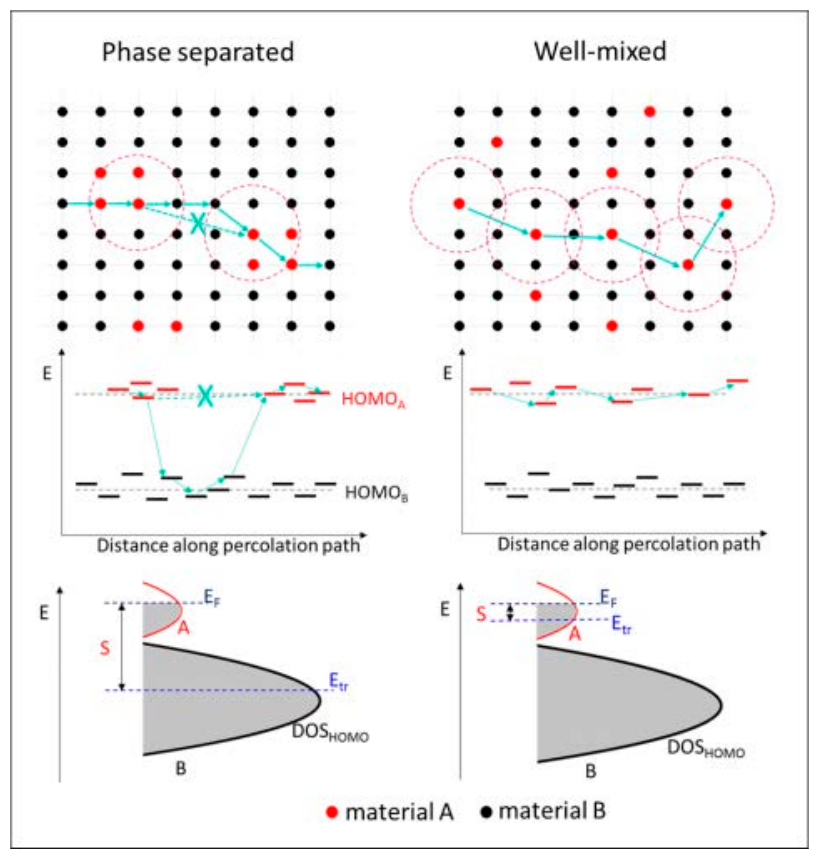

Figure 4.4 Schematic overview of (top) the site distribution in a 2-component blend (dashed circles indicate the typical hopping distance; green arrows indicate the hopping path); (middle) corresponding energy diagram; (bottom) density of states and characteristic energies, in (left) phase separated and (right) well-mixed morphologies with the same blend ratio. Materials A and $\mathrm{B}$ are indicated by red and black colors, respectively. Adapted with permission from Paper VII.

The starting assumption is that charge transport via localized sites in OSCs occurs by thermally activated tunneling, ${ }^{57}$ commonly described as hopping. When a small fraction of higher-lying HOMO material A mixes with material B, the hole conductivity in a VRH system is, according 
to percolation theory, ${ }^{22,72}$ governed by pathways that optimize the trade-off between hopping to sites that are near in energy (that statistically will not always be spatially nearby) and sites that are spatially near (that statistically will be further away in energy). For strongly phase separated morphologies, material A forms clusters distributed far away from each other in material B, see left panel in Figure 4.4. For such clusters the typical tunneling distance is much smaller than the typical inter-cluster distance. Therefore, the percolating pathway must contain sites from material B, i.e. they have to hop over a relatively large energy difference, corresponding to the HOMO energy difference between materials $\mathrm{A}$ and $\mathrm{B}$. Hence, the $E_{t r}$ shifts to the DOS of material B, while $E_{F}$ is still located on material A. Thus, this results in a high thermopower due to $S \propto E_{F}-E_{t r}$, as was also explained in Figure 4.2. Concomitantly, the conductivity will be low due to the isolated clusters acting as traps. This situation is comparable to a NNH hopping model in which charges have few possibilities to avoid energetically unfavorable hops. Note that Figure $4.3 \mathrm{~b}$ was calculated using a NNH kMC model.
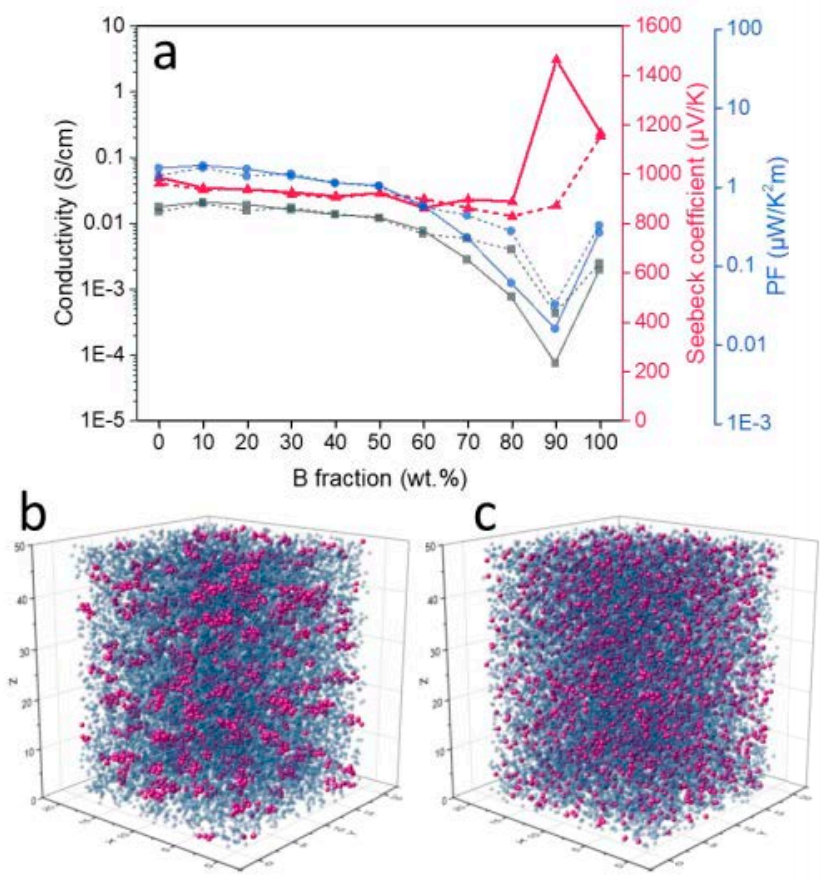

Figure 4.5 a. Conductivity, Seebeck coefficient and power factor vs. composition from VRH kinetic Monto Carlo simulations for $\mathrm{A}_{x}: \mathrm{B}_{1-x}$ mixtures with clusters (solid lines) and without clusters (dashed lines). b. and c. Typical morphologies used in the kinetic Monto Carlo simulations for $\mathrm{A}_{10}: \mathrm{B}_{90}$ mixtures $\mathrm{w} /$ and w/o clustering of the A component. Parameters used are the HOMO energy difference $\triangle E_{\text {НОМO }}=0.3 \mathrm{eV}$ and doping concentrations $c=10^{-3}, v_{0}=10^{-}$ ${ }^{13} \mathrm{~s}^{-1}, a_{N N}=1.8 \mathrm{~nm}, \sigma_{D O S}=75 \mathrm{meV}, T=300 \mathrm{~K}, \mathrm{HOMO}_{\mathrm{A}}-\mathrm{LUMO}_{\text {dopant }}=0.24 \mathrm{eV}, \alpha=2 \mathrm{e} 9 \mathrm{~nm}^{-}$ 
${ }^{1}$; correlation radius of the A compound clusters in units of $a_{N N} r_{C}=1.5$ or 0 . Adapted with permission from Paper VII.

For a well-mixed blend morphology, a percolating path can be formed that includes only A sites that are (more or less) randomly distributed in material B. The requirement for this to happen is that the typical tunneling distance is comparable or larger than the typical distance between sites of material A. Then, $E_{F}$ and $E_{t r}$ are both located on the DOS of material A, and the Seebeck coefficient and conductivity are hardly influenced by the blending with material B.

Kinetic Monte Carlo simulations confirm this proposed mechanism that morphology determines the behavior of the electrical conductivity and the Seebeck coefficient. Figure 4.5 shows the results from $\mathrm{kMC}$ simulations of VRH and typical used morphologies corresponding to $\mathrm{A}_{10}: \mathrm{B}_{90}$. Clearly, a peak in $S$ occurs for the clustered morphology that was generated by using a numerical annealing procedure. ${ }^{139,140}$
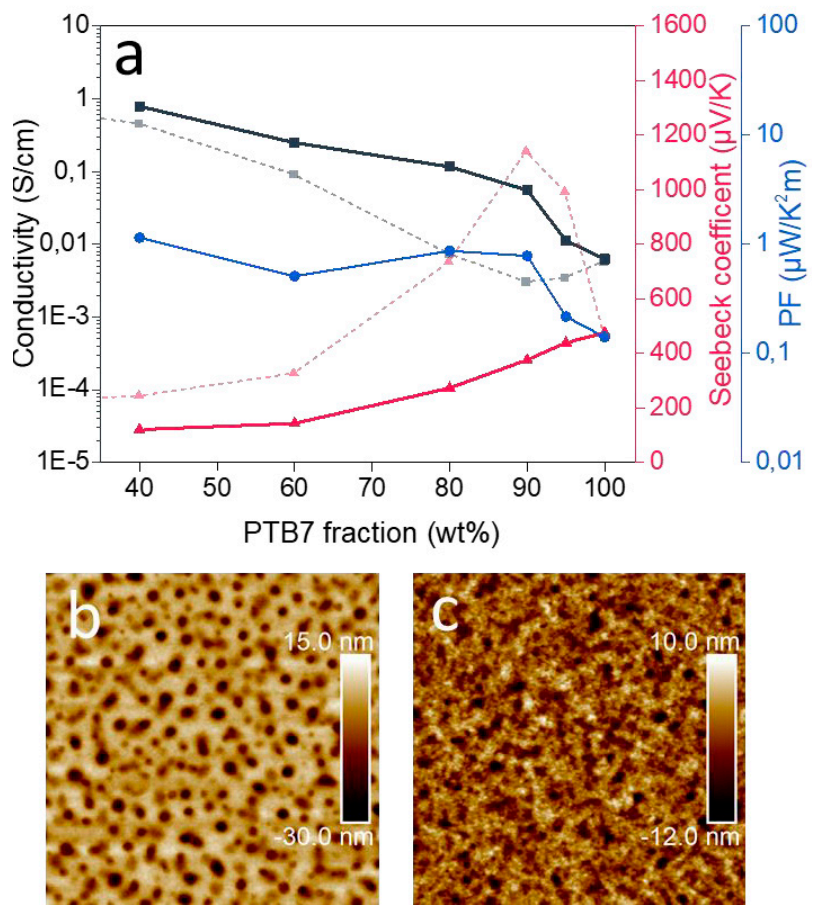

Figure 4.6 a. Conductivity, Seebeck coefficient and power factor for P3HT:PTB7 with 5\% DIO dependent on active layer composition. Dashed lines are data from Figure 4.3a, i.e. without DIO; AFM image for b. $\mathrm{P}_{3} \mathrm{HT}_{0.1}$ :PTB7 0.9 without DIO and c. with $5 \%$. All sizes $5 \times 5 \mu \mathrm{m}$. Adapted with permission from Paper VII.

We also experimentally examine the proposed relation between morphology and thermoelectric properties. Adding $5 \mathrm{v} \%$ DIO to the casting solution of P3HT:PTB7 blends significantly suppresses phase separation as seen in Figure 4.6 (b,c). The clear peak in $S$ occurring at $\mathrm{P}_{3} \mathrm{HT}_{10}: \mathrm{PTB}_{90}$ without DIO vanishes when the phase separated morphology is suppressed by 
the additive DIO. ${ }^{141,142}$ Further details on how we confirm the proposed mechanism from both simulations and experiments are given in Paper VII; In Paper V we find the same effect for ntype blends, although we could not fully explain the observed behavior at the time of writing. ${ }^{34}$

\subsection{Application potential}

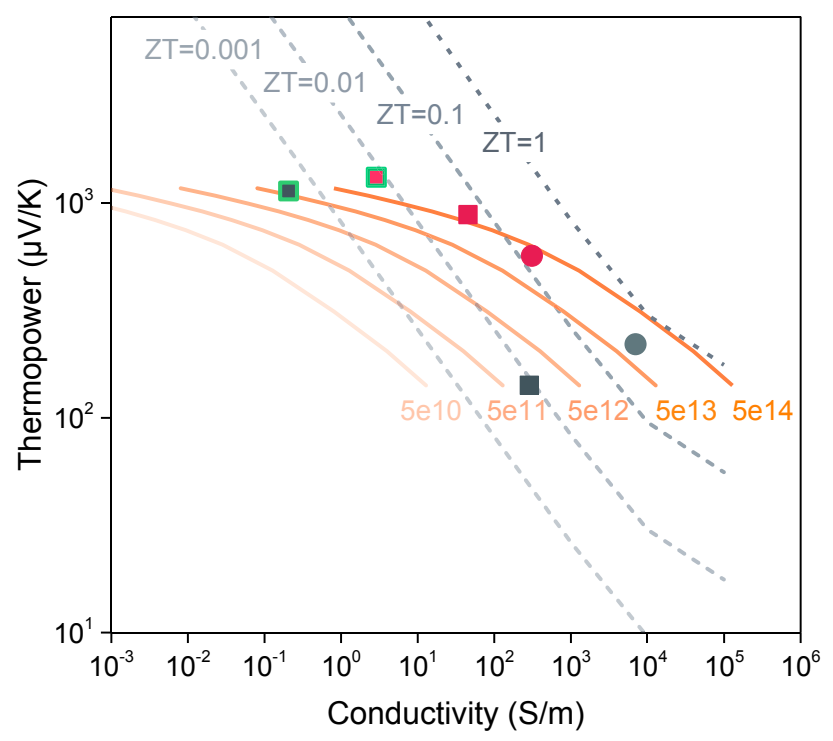

Figure 4.7 Contour plot parametric in the thermoelectric figure of merit ZT (dashed lines) at $300 \mathrm{~K}$ (Eq. 4.1) and thermopower vs. conductivity calculated from Model II (solid lines) with $v_{0}$ from $5 \times 10^{-10} \mathrm{~s}^{-1}$ to $5 \times 10^{-14} \mathrm{~s}^{-1}$, and other standard parameters: $a_{N N}=1.8 \mathrm{~nm}, \sigma_{D O S}=75$ $\mathrm{meV}, T=300 \mathrm{~K}, \alpha=2 \mathrm{e} 9 \mathrm{~nm}^{-1}$. The squares are from our work and show the highest-S data point (with green edge, $\mathrm{P}_{3} \mathrm{HT}_{10}: \mathrm{PTB}_{90}$ (gray) and $\mathrm{PCBM}_{1}: \mathrm{NDI}_{99}$ (red)), and the highest-PF data point (without green edge, pure P3HT (gray) and PCBM(red)). The red circle is for (ntype) N-DMBI-doped A-DCV-DPPTT $\left(\right.$ Ref. $^{38}$ ) and the gray circle is for (p-type) PEDOT-Tos (Ref. $\left.{ }^{8}\right)$.

Interest in organic thermoelectrics is growing dramatically in scientific community recently. It is therefore interesting to be able to predict the application potential for both n- and p-type OSC in organic thermoelectric applications. To this end, we reformulate the ZT expression to relate electrical conductivity to thermopower as follows:

$$
S=\sqrt{\frac{\kappa \cdot Z T}{\sigma T}}
$$


Here $\kappa=\kappa_{e}+\kappa_{l}$ as described in Chapter 1. In Figure 4.7 we plotted $S$ as a parametric function of conductivity for different ZT (dashed lines). Also plotted as orange lines are the relations between $\mathbf{S}$ and $\sigma$ as obtained from the VRH model introduced in Chapter 3 and Paper $\mathbf{V}$ for different values of the attempt-to-hop frequency. For each line, the doping concentration increases towards higher conductivity. The crossings of the two sets of lines therefore show that higher doping concentrations are expected to yield higher ZT for simple OSCs.

We took the reported records for both n-type and p-type thermoelectric materials from literature to test the model accuracy. ${ }^{8,31,38,34}$ Specifically, we plotted the n-type material N-DMBI-doped A-DCV-DPPTT from Ref. ${ }^{38}$ (red circle) and the p-type material PEDOT:ToS from Ref. ${ }^{8}$ (grey circle). The positions of these points on the plot with respect to the constant-ZT contours match excellently with the reported ZT values of $\sim 0.11$ and $\sim 0.25$, respectively.

Our best experimental results from p-type and n-type materials are shown as square symbol in Figure 4.7. When we assume that for the doped single (non-blended) OSCs, the thermoelectric properties are limited by the amount of doping we effectively get in, it should be possible to move along the orange lines and reach $\mathrm{ZT}=0.1$ or maybe even 1 for the $n$-type materials (red square). We note that it is not likely that the p-type model system P3HT (gray square) will ever reach relevant $\mathrm{ZT}$ as it already appears to sit close to the maximally obtainable doping concentration. ${ }^{31}$ For the blend systems, the high $S$ unavoidably comes at the price of lower conductivity, hence it is not likely that one can move along the orange line, so these compounds are likely constrained to applications where high thermopower is more important than high ZT. 


\section{Conclusion}

All the work included in this thesis shows that the density of states (DOS) is the starting point to understand the physics of, and to design materials for disordered organic thermoelectric devices. The different roles guest materials can have in multi-component systems (dopant or second semiconductor) makes that the guest(s) can give different contributions to the overall DOS. Combined, these determine the charge and energy transport by affecting the relative positions of the Fermi energy and the transport energy in the overall DOS.

DOS engineering is an effective way to tune the Seebeck coefficient by playing with composition in blended two-semiconductor systems. Apart from the energetics, one must also control the morphology to obtain the desired charge and energy transport. In all cases, a high achieved thermopower comes at the cost of conductivity, so the blending strategy is less suitable for high-power applications but rather for high-voltage, low-power applications. Nevertheless, both in simulations and in various experiments, we have shown that it is possible to increase the Seebeck coefficient without sacrificing electrical conductivity. Future research in this direction could be the experimental investigation of the actual hopping distance and how to control it.

Our findings suggest that n-type materials have more potential than p-type materials to achieve high ZT. However, due to (current) limitations of n-type materials and dopants in terms of availability and stability, n-type thermoelectrics are currently somewhat worse than p-types. New, high-mobility and air-stable n-type materials are necessary to develop complete high ZT thermoelectric generators. The next step in this direction should be to confirm in experiments that indeed the attempt-to-hop frequency of electrons is generally higher than that of holes. If so, the reason why electrons have a higher attempt-to-hop frequency should be understood physically.

It follows from our work that most disordered conjugated polymers contain a significant density of electron and hole traps. In contrast to previous work, we find that the trap energies are not constant but rather follow the corresponding HOMO or LUMO level at a constant offset of about $0.3-0.4 \mathrm{eV}$. The origin of the traps is still unclear. The next step is to figure out this origin, which is extremely important to material design and device optimization. 


\section{Acknowledgements}

This thesis was conducted in 2014-2018 at the Complex Materials and Devices group (CoMaDe) led by Martijn Kemerink, my supervisor, to whom I would like to express my deepest gratitude. Martijn, your knowledge is like Wikipedia and you always give me creative support and insightful suggestions to all my questions throughout my whole $\mathrm{PhD}$ period. I am very grateful for your endless patience and extreme kindness to tolerate my mistakes and teach me the physics as thorough as possible.

I would also like to express a deep sense of gratitude to my co-supervisor Ergang Wang, who introduced the opportunity for me to study here, and supported help in various ways for my scientific research. Also my sincere thanks to Zhaojun Li from Chalmers University of Technology, who synthesized and supported sufficient materials for me during my $\mathrm{PhD}$ period, besides the great help on device characterizations for me during my Master period in Beijing.

I would like to thank the China Scholarship Council (CSC) for financially supporting my study and living in Sweden.

Many thanks to Olof Andersson, who joined the CoMaDe group on the same day as I. I really appreciate all your help to make my life easy in Sweden like you borrowing your girlfriend horse cargo to help me when moving between apartments, always giving me a ride but so easily to park wrong places receiving a parking ticket. I very much appreciate that you have helped me move on when I were trapped in 'tough time' for several months. I would also like to thank all our CoMaDe crowds in the sequence I met: Nikolaos Felekidis, Hassan Abdalla, Indre Urbanavičiūte, Tim Cornelissen, Davood Abbaszadeh and Tanvi Upreti. Especially I would like to thank Hassan Abdalla for his collaborations with me and his all kinds of smart and maneuverable instrumental setups to make me carry on research work smoothly.

My gratitude also goes to Olle Inganäs for offering me the nice laboratory to fabricate all kinds of devices. I would like to thank the people we met in lab for the enjoyable time and all the help that I have received when I spent my lab work time there: Fengling Zhang, Feng Gao, Wanzhu Cai, Armantas Melianas, Thomas Österberg, Xing Xing, Luis Ever Aguirre, Yuxin Xia, Qingzhen Bian, Zhongcheng Yuan, Zaifang Li, Heyong Wang, Hongling Yu, Ke Zhou, Zhibo Yan, Liangqi Ouyang, Yuming Wang, Leiqiang Qian, Jonas Bergqvist, Fátima Ajjan.

I would also like to thank Mats Fahlman and Xian Jie Liu for helping me in experiments of ultraviolet photoemission spectroscopy. Xianjie is very nice to teach me how to read the measured spectrums.

Thanks to our administrators Anna-Maria Uhlin, Lena Martinsson, Jenny Ählström, and Emma Ljungkvist. All the administrative support allows me to mostly focus on science.

All my Chinese and non-Chinese friends in Linköping are also sincerely acknowledged.

Finally, I want to thank my family and especially my parents for supporting me all the years. 


\section{References}

1. Russ, B., Glaudell, A., Urban, J. J., Chabinyc, M. L. \& Segalman, R. A. Organic thermoelectric materials for energy harvesting and temperature control. Nat. Rev. Mater. 1, 16050 (2016).

2. Di, C., Xu, W. \& Zhu, D. Organic thermoelectrics for green energy. Natl. Sci. Rev. 3, 269-271 (2016).

3. Wei, Q., Mukaida, M., Kirihara, K., Naitoh, Y. \& Ishida, T. Polymer thermoelectric modules screen-printed on paper. RSC Adv. 4, 28802-28806 (2014).

4. Kroon, R. et al. Thermoelectric plastics: from design to synthesis, processing and structure-property relationships. Chem. Soc. Rev. 45, 6147-6164 (2016).

5. Bubnova, O. \& Crispin, X. Towards polymer-based organic thermoelectric generators. Energy Environ. Sci. 5, 9345-9362 (2012).

6. He, M., Qiu, F. \& Lin, Z. Towards high-performance polymer-based thermoelectric materials. Energy Environ. Sci. 6, 1352-1361 (2013).

7. Zhang Qian, Sun Yimeng, Xu Wei \& Zhu Daoben. Organic Thermoelectric Materials: Emerging Green Energy Materials Converting Heat to Electricity Directly and Efficiently. Adv. Mater. 26, 6829-6851 (2014).

8. Bubnova, O. et al. Optimization of the thermoelectric figure of merit in the conducting polymer poly(3,4-ethylenedioxythiophene). Nat. Mater. 10, 429-433 (2011).

9. Champier, D. Thermoelectric generators: A review of applications. Energy Convers. Manag. 140, 167-181 (2017).

10. He, W. et al. Recent development and application of thermoelectric generator and cooler. Appl. Energy 143, 1-25 (2015).

11. Singh, D. V. \& Pedersen, E. A review of waste heat recovery technologies for maritime applications. Energy Convers. Manag. 111, 315-328 (2016).

12. Zhang, Q., Sun, Y., Xu, W. \& Zhu, D. Organic Thermoelectric Materials: Emerging Green Energy Materials Converting Heat to Electricity Directly and Efficiently. Adv. Mater. 26, 6829-6851 (2014).

13. Tritt, T. M. Holey and Unholey Semiconductors. Science 283, 804-805 (1999).

14. DiSalvo, F. J. Thermoelectric Cooling and Power Generation. Science 285, 703-706 (1999).

15. Bahk, J.-H., Fang, H., Yazawa, K. \& Shakouri, A. Flexible thermoelectric materials and device optimization for wearable energy harvesting. J Mater Chem C 3, (2015).

16. Cahill, D. G. \& Pohl, R. O. Thermal conductivity of amorphous solids above the plateau. Phys. Rev. B 35, 4067-4073 (1987).

17. Kalia, S., Mahajan, A., Ghansyam, C. G. \& Bedi, R. K. Mobility modulation in low carrier concentration organic semiconducting thin films by varying disorder parameters. J. Appl. Phys. 121, 225501 (2017).

18. Zhou, X. et al. Enhanced Hole Injection into Amorphous Hole-Transport Layers of Organic Light-Emitting Diodes Using Controlled p-Type Doping. Adv. Funct. Mater. 11, 310-314 (2001).

19. Loiudice, A., Rizzo, A., Biasiucci, M. \& Gigli, G. Bulk Heterojunction versus Diffused Bilayer: The Role of Device Geometry in Solution p-Doped Polymer-Based Solar Cells. J. Phys. Chem. Lett. 3, 1908-1915 (2012).

20. Zhang, Y. et al. Molecular Doping Enhances Photoconductivity in Polymer Bulk Heterojunction Solar Cells. Adv. Mater. 25, 7038-7044 (2013).

21. Yim, K.-H. et al. Controlling Electrical Properties of Conjugated Polymers via a Solution-Based p-Type Doping. Adv. Mater. 20, 3319-3324 (2008). 
22. Baranovskii, S. D. Theoretical description of charge transport in disordered organic semiconductors. Phys. Status Solidi B 251, 487-525 (2014).

23. Zuo, G., Liu, X., Fahlman, M. \& Kemerink, M. High Seebeck Coefficient in Mixtures of Conjugated Polymers. Adv. Funct. Mater. 28, 1703280 (2018)

24. Germs, W. C. et al. Charge transport in amorphous InGaZnO thin-film transistors. Phys. Rev. B 86, 155319 (2012).

25. Kumar, G. S., Vandersande, J. W., Klitsner, T., Pohl, R. O. \& Slack, G. A. Lowtemperature heat transport by charge carriers in doped semiconductors. Phys. Rev. B 31, 2157-2162 (1985)

26. D G Cahill \& Pohl, and R. O. Lattice Vibrations and Heat Transport in Crystals and Glasses. Annu. Rev. Phys. Chem. 39, 93-121 (1988).

27. Weathers, A. et al. Significant Electronic Thermal Transport in the Conducting Polymer Poly(3,4-ethylenedioxythiophene). Adv. Mater. 27, 2101-2106 (2015).

28. Kang, S. D. \& Snyder, G. J. Charge-transport model for conducting polymers. Nat. Mater. 16, 252-257 (2017).

29. Glassbrenner, C. J. \& Slack, G. A. Thermal Conductivity of Silicon and Germanium from $3{ }^{\circ} \mathrm{K}$ to the Melting Point. Phys. Rev. 134, A1058-A1069 (1964).

30. Tritt, T. M. \& Subramanian, M. A. Thermoelectric Materials, Phenomena, and Applications: A Bird's Eye View. MRS Bull. 31, 188-198 (2006).

31. Zuo, G., Andersson, O., Abdalla, H. \& Kemerink, M. High thermoelectric power factor from multilayer solution-processed organic films. Appl. Phys. Lett. 112, 083303 (2018).

32. Venkateshvaran, D. et al. Approaching disorder-free transport in high-mobility conjugated polymers. Nature 515, 384-388 (2014).

33. Zhang, F. et al. Modulated Thermoelectric Properties of Organic Semiconductors Using Field-Effect Transistors. Adv. Funct. Mater. 25, 3004-3012 (2015).

34. Zuo, G., Li, Z., Wang, E. \& Kemerink, M. High Seebeck Coefficient and Power Factor in n-Type Organic Thermoelectrics. Adv. Electron. Mater. 4, 1700501 (2017).

35. Snyder, G. J. \& Snyder, A. H. Figure of merit ZT of a thermoelectric device defined from materials properties. Energy Environ. Sci. 10, 2280-2283 (2017).

36. Patel, S. N. et al. Morphology controls the thermoelectric power factor of a doped semiconducting polymer. Sci. Adv. 3, e1700434 (2017).

37. Wei, P. et al. 2-(2-Methoxyphenyl)-1,3-dimethyl-1H-benzoimidazol-3-ium Iodide as a New Air-Stable n-Type Dopant for Vacuum-Processed Organic Semiconductor Thin Films. J. Am. Chem. Soc. 134, 3999-4002 (2012).

38. Huang, D. et al. Conjugated-Backbone Effect of Organic Small Molecules for n-Type Thermoelectric Materials with ZT over 0.2. J. Am. Chem. Soc. 139, 13013-13023 (2017).

39. Kroon, R. et al. Thermoelectric plastics: from design to synthesis, processing and structure-property relationships. Chem. Soc. Rev. 45, 6147-6164 (2016).

40. Liu, J. et al. N-Type Organic Thermoelectrics: Improved Power Factor by Tailoring Host-Dopant Miscibility. Adv. Mater. 29, 1701641 (2017).

41. Menke, T. et al. A comparison of two air-stable molecular n-dopants for C60. Org. Electron. 13, 3319-3325 (2012).

42. Shi, K. et al. Toward High Performance n -Type Thermoelectric Materials by Rational Modification of BDPPV Backbones. J. Am. Chem. Soc. 137, 6979-6982 (2015).

43. Wei, P., Oh, J. H., Dong, G. \& Bao, Z. Use of a 1H-Benzoimidazole Derivative as an nType Dopant and To Enable Air-Stable Solution-Processed n-Channel Organic ThinFilm Transistors. J. Am. Chem. Soc. 132, 8852-8853 (2010). 
44. Glaudell Anne M., Cochran Justin E., Patel Shrayesh N. \& Chabinyc Michael L. Impact of the Doping Method on Conductivity and Thermopower in Semiconducting Polythiophenes. Adv. Energy Mater. 5, 1401072 (2014).

45. Li Zaifang et al. A Free-Standing High-Output Power Density Thermoelectric Device Based on Structure-Ordered PEDOT:PSS. Adv. Electron. Mater. 4, 1700496 (2018).

46. Kroon Renee et al. Bulk Doping of Millimeter-Thick Conjugated Polymer Foams for Plastic Thermoelectrics. Adv. Funct. Mater. 27, 1704183 (2017).

47. Nielsen, C. B., Holliday, S., Chen, H.-Y., Cryer, S. J. \& McCulloch, I. Non-Fullerene Electron Acceptors for Use in Organic Solar Cells. Acc. Chem. Res. 48, 2803-2812 (2015).

48. Grimsdale, A. C., Leok Chan, K., Martin, R. E., Jokisz, P. G. \& Holmes, A. B. Synthesis of Light-Emitting Conjugated Polymers for Applications in Electroluminescent Devices. Chem. Rev. 109, 897-1091 (2009).

49. Zhao, Y., Guo, Y. \& Liu, Y. 25th Anniversary Article: Recent Advances in n-Type and Ambipolar Organic Field-Effect Transistors. Adv. Mater. 25, 5372-5391 (2013).

50. Molecular Orbital Theory. Mol. Orbitals Org. Chem. React. (2009).

51. The Structures of Organic Molecules. Mol. Orbitals Org. Chem. React. (2009).

52. Clarke, T. M. \& Durrant, J. R. Charge Photogeneration in Organic Solar Cells. Chem. Rev. 110, 6736-6767 (2010).

53. Glaudell, A. M., Cochran, J. E., Patel, S. N. \& Chabinyc, M. L. Impact of the Doping Method on Conductivity and Thermopower in Semiconducting Polythiophenes. Adv. Energy Mater. 5, 1401072 (2015).

54. Elgrishi, N. et al. A Practical Beginner's Guide to Cyclic Voltammetry. J. Chem. Educ. 95, 197-206 (2018).

55. Himpsel F. J. Ultraviolet Photoelectron Spectroscopy. Charact. Mater. (2002).

56. Bredas, J.-L. Mind the gap! Mater. Horiz. 1, 17-19 (2014).

57. Bässler H. Charge Transport in Disordered Organic Photoconductors a Monte Carlo Simulation Study. Phys. Status Solidi B 175, 15-56 (2006).

58. Graupner, W., Leditzky, G., Leising, G. \& Scherf, U. Shallow and deep traps in conjugated polymers of high intrachain order. Phys. Rev. B 54, 7610-7613 (1996).

59. P. Nikiforov, M. et al. Detection and role of trace impurities in high-performance organic solar cells. Energy Environ. Sci. 6, 1513-1520 (2013).

60. Meier, M., Karg, S., Zuleeg, K., Brütting, W. \& Schwoerer, M. Determination of trapping parameters in poly(p-phenylenevinylene) light-emitting devices using thermally stimulated currents. J. Appl. Phys. 84, 87-92 (1998).

61. Vissenberg, M. C. J. M. \& Matters, M. Theory of the field-effect mobility in amorphous organic transistors. Phys. Rev. B 57, 12964-12967 (1998).

62. Mihailetchi, V. D., Blom, P. W. M., Hummelen, J. C. \& Rispens, M. T. Cathode dependence of the open-circuit voltage of polymer:fullerene bulk heterojunction solar cells. J. Appl. Phys. 94, 6849-6854 (2003).

63. Blakesley, J. C. \& Neher, D. Relationship between energetic disorder and open-circuit voltage in bulk heterojunction organic solar cells. Phys. Rev. B 84, 075210 (2011).

64. Boix, P. P. et al. Determination of gap defect states in organic bulk heterojunction solar cells from capacitance measurements. Appl. Phys. Lett. 95, 233302 (2009).

65. Blakesley, J. C. et al. Time-of-flight measurements and vertical transport in a high electron-mobility polymer. Appl. Phys. Lett. 99, 183310 (2011).

66. Lange, I. et al. Band Bending in Conjugated Polymer Layers. Phys. Rev. Lett. 106, 216402 (2011). 
67. Yogev, S., Halpern, E., Matsubara, R., Nakamura, M. \& Rosenwaks, Y. Direct measurement of density of states in pentacene thin film transistors. Phys. Rev. B 84, 165124 (2011).

68. Tanase, C., Meijer, E. J., Blom, P. W. M. \& de Leeuw, D. M. Unification of the Hole Transport in Polymeric Field-Effect Transistors and Light-Emitting Diodes. Phys. Rev. Lett. 91, 216601 (2003).

69. Miller, A. \& Abrahams, E. Impurity Conduction at Low Concentrations. Phys. Rev. 120, 745-755 (1960).

70. Pasveer, W. F. et al. Unified Description of Charge-Carrier Mobilities in Disordered Semiconducting Polymers. Phys. Rev. Lett. 94, 206601 (2005).

71. Cottaar, J., Koster, L. J. A., Coehoorn, R. \& Bobbert, P. A. Scaling Theory for Percolative Charge Transport in Disordered Molecular Semiconductors. Phys. Rev. Lett. 107, 136601 (2011).

72. Vissenberg, M. C. J. M. \& Matters, M. Theory of the field-effect mobility in amorphous organic transistors. Phys. Rev. B 57, 12964-12967 (1998).

73. Ries, B., Bässler, H., Grünewald, M. \& Movaghar, B. Monte Carlo study of relaxation and diffusion in glassy systems. Phys. Rev. B 37, 5508-5517 (1988).

74. Ambegaokar, V., Halperin, B. I. \& Langer, J. S. Hopping Conductivity in Disordered Systems. Phys. Rev. B 4, 2612-2620 (1971).

75. Hartenstein, B. \& Bässler, H. Transport energy for hopping in a Gaussian density of states distribution. J. Non-Cryst. Solids 190, 112-116 (1995).

76. Kuik Martijn et al. 25th Anniversary Article: Charge Transport and Recombination in Polymer Light-Emitting Diodes. Adv. Mater. 26, 512-531 (2014).

77. Coehoorn, R., Pasveer, W. F., Bobbert, P. A. \& Michels, M. A. J. Charge-carrier concentration dependence of the hopping mobility in organic materials with Gaussian disorder. Phys. Rev. B 72, 155206 (2005).

78. Wetzelaer, G.-J. A. H. et al. Asymmetric electron and hole transport in a high-mobility n-type conjugated polymer. Phys. Rev. B 86, 165203 (2012).

79. Kemerink, M. et al. Temperature- and density-dependent channel potentials in highmobility organic field-effect transistors. Phys. Rev. B 80, 115325 (2009).

80. Nicolai, H. T. et al. Unification of trap-limited electron transport in semiconducting polymers. Nat. Mater. 11, 882-887 (2012).

81. de Leeuw, D. M., Simenon, M. M. J., Brown, A. R. \& Einerhand, R. E. F. Stability of ntype doped conducting polymers and consequences for polymeric microelectronic devices. Synth. Met. 87, 53-59 (1997).

82. Mark, P. \& Helfrich, W. Space-Charge-Limited Currents in Organic Crystals. J. Appl. Phys. 33, 205-215 (1962).

83. Rose, A. Space-Charge-Limited Currents in Solids. Phys. Rev. 97, 1538-1544 (1955).

84. Moiz, S. A., Khan, I. A., Younis, W. A. \& S.Karimov, K. Space Charge-Limited Current Model for Polymers. Conduct. Polym. (2016).

85. Nicolai, H. T., Mandoc, M. M. \& Blom, P. W. M. Electron traps in semiconducting polymers: Exponential versus Gaussian trap distribution. Phys. Rev. B 83, 195204 (2011).

86. Zuo, G. et al. Molecular Doping and Trap Filling in Organic Semiconductor Host-Guest Systems. J. Phys. Chem. C 121, 7767-7775 (2017).

87. Shang, Z. et al. Trade-Off between Trap Filling, Trap Creation, and Charge Recombination Results in Performance Increase at Ultralow Doping Levels in Bulk Heterojunction Solar Cells. Adv. Energy Mater. 6, (2016). 
88. Blochwitz, J., Pfeiffer, M., Fritz, T. \& Leo, K. Low voltage organic light emitting diodes featuring doped phthalocyanine as hole transport material. Appl. Phys. Lett. 73, 729-731 (1998).

89. Lüssem, B. et al. Doped Organic Transistors. Chem. Rev. 116, 13714-13751 (2016).

90. Hong, C. T. et al. Effect of film thickness and crystallinity on the thermoelectric properties of doped P3HT films. RSC Adv. 5, 11385-11391 (2015).

91. Scholes, D. T. et al. Overcoming Film Quality Issues for Conjugated Polymers Doped with F4TCNQ by Solution Sequential Processing: Hall Effect, Structural, and Optical Measurements. J. Phys. Chem. Lett. 6, 4786-4793 (2015).

92. Xuan, Y. et al. Thermoelectric properties of conducting polymers: The case of poly(3hexylthiophene). Phys. Rev. B 82, 115454 (2010).

93. Wang, C., Duong, D. T., Vandewal, K., Rivnay, J. \& Salleo, A. Optical measurement of doping efficiency in poly(3-hexylthiophene) solutions and thin films. Phys. Rev. B 91, 085205 (2015).

94. Duong, D. T., Wang, C., Antono, E., Toney, M. F. \& Salleo, A. The chemical and structural origin of efficient p-type doping in P3HT. Org. Electron. 14, 1330-1336 (2013).

95. Scholes, D. T. et al. Overcoming Film Quality Issues for Conjugated Polymers Doped with F 4 TCNQ by Solution Sequential Processing: Hall Effect, Structural, and Optical Measurements. J. Phys. Chem. Lett. 6, 4786-4793 (2015).

96. Aziz, E. F. et al. Localized Charge Transfer in a Molecularly Doped Conducting Polymer. Adv. Mater. 19, 3257-3260 (2007).

97. Deschler, F. et al. Imaging of morphological changes and phase segregation in doped polymeric semiconductors. Synth. Met. 199, 381-387 (2015).

98. Gao, J., Niles, E. T. \& Grey, J. K. Aggregates Promote Efficient Charge Transfer Doping of Poly(3-hexylthiophene). J. Phys. Chem. Lett. 4, 2953-2957 (2013).

99. Hwang, S., Potscavage, W. J., Nakamichi, R. \& Adachi, C. Processing and doping of thick polymer active layers for flexible organic thermoelectric modules. Org. Electron. 31, 31-40 (2016).

100. Hynynen, J., Kiefer, D. \& Müller, C. Influence of crystallinity on the thermoelectric power factor of P3HT vapour-doped with F4TCNQ. RSC Adv. 8, 1593-1599 (2018).

101. Sakiyama, S., Mizutani, N. \& Fujita, K. Controllable p- and n-type doping of poly[2methoxy-5-(2'-methyl-hexyloxy)-p-phenylenevinylene] films prepared by evaporative spray deposition using ultradilute solution. Jpn. J. Appl. Phys. 55, 04EL03 (2016).

102. Gao, W. \& Kahn, A. Controlled p-doping of zinc phthalocyanine by coevaporation with tetrafluorotetracyanoquinodimethane: A direct and inverse photoemission study. Appl. Phys. Lett. 79, 4040-4042 (2001).

103. Irwin, M. D., Buchholz, D. B., Hains, A. W., Chang, R. P. H. \& Marks, T. J. p-Type semiconducting nickel oxide as an efficiency-enhancing anode interfacial layer in polymer bulk-heterojunction solar cells. Proc. Natl. Acad. Sci. 105, 2783-2787 (2008).

104. Sun, J. et al. Simultaneous Increase in Seebeck Coefficient and Conductivity in a Doped Poly(alkylthiophene) Blend with Defined Density of States. Macromolecules 43, 28972903 (2010).

105. Ouyang, J. "Secondary doping" methods to significantly enhance the conductivity of PEDOT:PSS for its application as transparent electrode of optoelectronic devices. Displays 34, 423-436 (2013).

106. Pathak, C. S., Singh, J. P. \& Singh, R. Effect of dimethyl sulfoxide on the electrical properties of PEDOT:PSS/n-Si heterojunction diodes. Curr. Appl. Phys. 15, 528-534 (2015). 
107. Liu, Q. et al. Optical anisotropy in solvent-modified poly(3,4-ethylenedioxythiophene): poly(styrenesulfonic acid) and its effect on the photovoltaic performance of crystalline silicon/organic heterojunction solar cells. Appl. Phys. Lett. 102, 243902 (2013).

108. Ouyang, J. et al. On the mechanism of conductivity enhancement in poly $(3,4-$ ethylenedioxythiophene):poly(styrene sulfonate) film through solvent treatment. Polymer 45, 8443-8450 (2004).

109. Yan, H. \& Okuzaki, H. Effect of solvent on PEDOT/PSS nanometer-scaled thin films: XPS and STEM/AFM studies. Synth. Met. 159, 2225-2228 (2009).

110. Reenen, S. van \& Kemerink, M. Light-Emitting Electrochemical Cells: Mechanisms and Formal Description. in Light-Emitting Electrochemical Cells 3-45 (Springer, Cham, 2017).

111. Yuen, J. D. et al. Electrochemical Doping in Electrolyte-Gated Polymer Transistors. J. Am. Chem. Soc. 129, 14367-14371 (2007).

112. Herlogsson L. et al. Low-Voltage Polymer Field-Effect Transistors Gated via a Proton Conductor. Adv. Mater. 19, 97-101 (2007).

113. Pei, Q., Yu, G., Zhang, C., Yang, Y. \& Heeger, A. J. Polymer Light-Emitting Electrochemical Cells. Science 269, 1086-1088 (1995).

114. Pei, Q., Yang, Y., Yu, G., Zhang, C. \& Heeger, A. J. Polymer Light-Emitting Electrochemical Cells: In Situ Formation of a Light-Emitting p-n Junction. J. Am. Chem. Soc. 118, 3922-3929 (1996).

115. Sosorev, A. Y. \& Paraschuk, D. Y. Charge-Transfer Complexes of Conjugated Polymers. Isr. J. Chem. 54, 650-673 (2014).

116. Pingel, P. \& Neher, D. Comprehensive picture of p-type doping of P3HT with the molecular acceptor F4TCNQ. Phys. Rev. B 87, 115209 (2013).

117. Wang, C., Duong, D. T., Vandewal, K., Rivnay, J. \& Salleo, A. Optical measurement of doping efficiency in poly(3-hexylthiophene) solutions and thin films. Phys. Rev. B 91, 085205 (2015).

118. Aziz E. F. et al. Localized Charge Transfer in a Molecularly Doped Conducting Polymer. Adv. Mater. 19, 3257-3260 (2007).

119. Kiefer, D. et al. A Solution-Doped Polymer Semiconductor:Insulator Blend for Thermoelectrics. Adv. Sci. 4, 1600203 (2017).

120. Liu Jian et al. Enhancing Molecular n-Type Doping of Donor-Acceptor Copolymers by Tailoring Side Chains. Adv. Mater. 30, 1704630 (2018).

121. Hamidi-Sakr, A. et al. A Versatile Method to Fabricate Highly In-Plane Aligned Conducting Polymer Films with Anisotropic Charge Transport and Thermoelectric Properties: The Key Role of Alkyl Side Chain Layers on the Doping Mechanism. Adv. Funct. Mater. 27, 1700173 (2017).

122. Pingel, P., Schwarzl, R. \& Neher, D. Effect of molecular p-doping on hole density and mobility in poly(3-hexylthiophene). Appl. Phys. Lett. 100, 143303 (2012).

123. Tietze, M. L., Pahner, P., Schmidt, K., Leo, K. \& Lüssem, B. Doped Organic Semiconductors: Trap-Filling, Impurity Saturation, and Reserve Regimes. Adv. Funct. Mater. 25, 2701-2707 (2015).

124. Arkhipov, V. I., Emelianova, E. V., Heremans, P. \& Bässler, H. Analytic model of carrier mobility in doped disordered organic semiconductors. Phys. Rev. B 72, 235202 (2005).

125. Arkhipov, V. I., Heremans, P., Emelianova, E. V. \& Bässler, H. Effect of doping on the density-of-states distribution and carrier hopping in disordered organic semiconductors. Phys. Rev. B 71, 045214 (2005). 
126. Shimotani, H., Diguet, G. \& Iwasa, Y. Direct comparison of field-effect and electrochemical doping in regioregular poly(3-hexylthiophene). Appl. Phys. Lett. 86, 022104 (2005).

127. Abdalla, H., Zuo, G. \& Kemerink, M. Range and energetics of charge hopping in organic semiconductors. Phys. Rev. B 96, 241202-241202 (2017).

128. Ihnatsenka, S., Crispin, X. \& Zozoulenko, I. V. Understanding hopping transport and thermoelectric properties of conducting polymers. Phys. Rev. B 92, 035201 (2015).

129. Chaikin, P. M. \& Beni, G. Thermopower in the correlated hopping regime. Phys. Rev. $B$ 13, 647-651 (1976).

130. Lu, N., Li, L. \& Liu, M. Universal carrier thermoelectric-transport model based on percolation theory in organic semiconductors. Phys. Rev. B 91, 195205 (2015).

131. Grünewald M. \& Thomas P. Percolation and Effective Medium Theory of Conductivity and Thermopower in the Hopping Transport Regime. Phys. Status Solidi B 93, K17-K20 (2006).

132. Schmechel, R. Hopping transport in doped organic semiconductors: A theoretical approach and its application to p-doped zinc-phthalocyanine. J. Appl. Phys. 93, 46534660 (2003).

133. Shi, W., Zhao, T., Xi, J., Wang, D. \& Shuai, Z. Unravelling Doping Effects on PEDOT at the Molecular Level: From Geometry to Thermoelectric Transport Properties. J. Am. Chem. Soc. 137, 12929-12938 (2015).

134. Xuan, Y. et al. Thermoelectric properties of conducting polymers: The case of poly(3hexylthiophene). Phys. Rev. B 82, 115454 (2010).

135. Mahan, G. D. \& Sofo, J. O. The best thermoelectric. Proc. Natl. Acad. Sci. 93, 74367439 (1996).

136. Lee, J. K. et al. Processing Additives for Improved Efficiency from Bulk Heterojunction Solar Cells. J. Am. Chem. Soc. 130, 3619-3623 (2008).

137. Liu, Y. et al. Aggregation and morphology control enables multiple cases of highefficiency polymer solar cells. Nat. Commun. 5, ncomms6293 (2014).

138. Park, S. H. et al. Bulk heterojunction solar cells with internal quantum efficiency approaching 100\%. Nat. Photonics 3, nphoton.2009.69 (2009).

139. Peumans, P., Uchida, S. \& Forrest, S. R. Efficient bulk heterojunction photovoltaic cells using small-molecular-weight organic thin films. Nature 425, nature01949 (2003).

140. Marsh, R. A., Groves, C. \& Greenham, N. C. A microscopic model for the behavior of nanostructured organic photovoltaic devices. J. Appl. Phys. 101, 083509 (2007).

141. Lou, S. J. et al. Effects of Additives on the Morphology of Solution Phase Aggregates Formed by Active Layer Components of High-Efficiency Organic Solar Cells. J. Am. Chem. Soc. 133, 20661-20663 (2011).

142. Chen, Y., Zhang, S., Wu, Y. \& Hou, J. Molecular Design and Morphology Control Towards Efficient Polymer Solar Cells Processed using Non-aromatic and Nonchlorinated Solvents. Adv. Mater. 26, 2744-2749 (2014). 


\section{Appendix}

$\boldsymbol{a}_{N N \text { : inter-site distance }}$

A-DCV-DPPTT: aromatic-dicyanovinyl-dipyrrolo[3,4-c]pyrrole-1,4-iylidene)bis(thieno[3,2b]thiophene

AFM: atomic force microscopy

BEDT-TTF: bis(ethylenedithia)tetrathiafulvalene

$\mathbf{B i}_{2} \mathbf{T e}_{3}$ : bismuth telluride

CP: conjugated polymer

$\mathbf{C r}_{2}$ (hpp)4: chromium, hpp, the anion of 1,3,4,6,7,8-hexahydro-2H-pyrimido[1,2-a]pyrimidine

CsCO3: carbonic acid dicesium

CTC: hybrid charge transfer complex

CV: cyclic voltammetry

DIO: 1,8-diiodooctane

DMSO: dimetylsulfoxid

DOS: density of states

$\boldsymbol{e}$ : elementary charge

EA: electron affinity

$\boldsymbol{E}_{\boldsymbol{F}}$ : Fermi energy

EG: ethylene glycol

$\boldsymbol{E}_{\boldsymbol{t}}$ : depth of trap energy

$\boldsymbol{E}_{t r}:$ transport energy

$\boldsymbol{E}_{\infty}$ : equilibration energy

$\mathrm{FeCl}_{3}$ : ferric chloride

F2TCNQ: 2,5-difluoro-7,7,8,8-tetracyanoquinodimethane

F4TCNQ: tetrafluoro-7,7,8,8-tetracyanoquinodimethane

F6TCNQ: 7,7,8,8-Tetracyanoquinodimethane

HOMO: the highest occupied molecular orbital 
ICT: integer charge transfer

IP: ionization potential

$J-V$ : current-voltage

$\boldsymbol{\kappa}$ : thermal conductivity

$\boldsymbol{k}_{\boldsymbol{B}}$ : Boltzmann constant

kMC: kinetic Monte Carlo

LCD: liquid-crystal display

LEC: light-emitting electrochemical cell

LUMO: lowest unoccupied molecular orbital

$\mu$ : mobility

MEH-PPV: poly[2-methoxy-5-(2'-ethylhexyloxy)-p-phenylene vinylene]

$\mathbf{n}_{\text {int }}$ : intrinsic charge density

N2200: poly[N,N'-bis(2-octyldodecyl)-1,4,5,8-naphthalenedicarboximide-6-diyl]-alt-5,5'(2,2'-bithiophene)]

N-DMBI: (4-(1,3- dimethyl-2,3-dihydro-1H-benzoimidazol-2-yl)phenyl)dimethylamine

N-DPBI: 4-(1,3-Dimethyl-2,3-dihydro-1H-benzoimidazol-2-yl)-N,N-diphenylaniline

NOPF6: nitrosium hexafluorophosphate

$N_{\text {dope }}$ : dopant density

$N_{t}$ : trap density

NNH: nearest neighbor happoing

OTE: organic thermoelectrics

OPV: organic solar cells

OLED: organic light-emitting devices

OFET: organic field effect transistor

P3HT: poly(3-hexylthiophene-2,5-diyl)

PBTTT: poly[2,5-bis(3-tetradecylthiophen-2-yl)thieno[3,2-b]thiophene]

PCBM: [6,6]-phenyl $\mathrm{C}_{61}$ butyric acid methyl ester or [6,6]-phenyl $\mathrm{C}_{71}$ butyric acid methyl ester 
PCPDTBT: poly[2,6-(4,4-bis-(2-ethylhexyl)-4H-cyclopenta [2,1-b;3,4-b']dithiophene)-alt4,7(2,1,3-benzothiadiazole)]

PEDOT:PSS: poly(2,3-dihydrothieno-1,4-dioxin)-poly(styrenesulfonate)

PEO: polyethyleneoxide

PEG-1: poly(3,4-ethylenedioxythiophene)-block-poly(ethylene glycol)

PF: power factor

PTB7: poly(\{4,8-bis[(2-ethylhexyl)oxy]benzo[1,2-b:4,5-b']dithiophene-2,6-diyl $\}\{3-$ fluoro-2[(2-ethylhexyl)carbonyl]thieno[3,4-b]thiophenediyl $\})$

$\mathbf{R}_{\mathbf{q}}$ : morphology roughness

\section{Ru(terpy)2:}

$\boldsymbol{\sigma}$ : electrical conductivity

$\boldsymbol{\sigma}_{\boldsymbol{D} \boldsymbol{O} \boldsymbol{s}}$ : energetic disorder of Gaussian shaped density of states

SCLC: space-charge-limited-current

S: Seebeck coefficient (thermopower)

Si: silicon

T: temperature

TDAE: tetrakis(dimethylamino)ethylene

TEG: thermoelectric generator

TQ1: poly[[2,3-bis(3-octyloxyphenyl)-5,8-quinoxalinediyl]-2,5-thiophenediyl]

Tos: tosylate

UPS: ultraviolet photoelectron spectroscopy

$\boldsymbol{v}_{\mathbf{0}}$ : attempt-to-hopping frequency

VRH: variable range hopping

ZT: figure of merit 


\section{Papers}

The papers associated with this thesis have been removed for copyright reasons. For more details about these see:

http://urn.kb.se/resolve?urn=urn:nbn:se:liu:diva-147778 